\title{
BISPHOSPHONATE-RELATED OSTEONECROSIS OF THE JAW
}

A DISSERTATION

SUBMITTED TO THE FACULTY OF THE GRADUATE SCHOOL OF THE UNVERSITY OF MINNESOTA

BY

VIVEK THUMBIGERE MATH

IN PARTIAL FULFILLMENT OF THE REQUIREMENTS

FOR THE DEGREE OF DOCTOR OF PHILOSOPHY

RAJARAM GOPALAKRISHNAN B.D.S., PH.D.

ADVISER

May 2013 
(C) Vivek Thumbigere Math 2013 


\section{ACKNOWLEDGEMENTS}

I would like to thank many people who have contributed to my learning. First and foremost, I would like to thank my mentor, Dr. Rajaram Gopalakrishnan, whose unconditional support has been paramount in providing me a sound research experience during my graduate studies. He has encouraged me to grow as an independent thinker and scientist. Truly, he has been more of a friend than a mentor.

I would also like to thank the members of my examining committee, Dr. Arkadiusz Dudek, Dr. Kim Mansky, Dr. William Toscano, and Dr. Joel Rudney. To each of them, I owe a great debt of gratitude for their patience, inspiration and friendship. Their insightful comments and constructive criticisms at different stages of my research were thought provoking and helped me focus on my ideas. I am deeply indebted to Dr. Dudek for supporting me during the initial stages of my graduate studies. Equally important have been Dr. Ma'Ann Sabino, Dr. David Basi, and Dr. Bryan Michalowicz. They have been a great source of intellectual support and critical advice. I am grateful to Dr. Michalowicz for holding me to a high research standard.

I also want to thank my lab members, Ann Emery, Julio Sotillo Rodriguez, Lan Pham, Raphael Huntley, and Dr. Eric Jensen for helping me with my experiments and for their friendship. Likewise, the faculty and staff of the Oral Pathology division have been extremely helpful during my graduate studies. 
I am also indebted to the faculty members and staff of the Oral Biology program. I would like to thank Dr. Edward Combe for providing me an opportunity to enroll in the Oral Biology graduate program. I have and always will admire Dr. Mark Herzberg for his wisdom, patience, and immense competency. He is an inspiration to aim for new objectives.

I would like to acknowledge the faculty members and staff of the Periodontology division. Particularly, I thank Dr. James Hinrichs, Dr. Larry Wolff, Dr. Massimo Costalonga, Dr. Satya Molleti, Dr. Deborah Johnson, Terri Abdelrahman, and Lisa Durkot for shaping and directing my career in Periodontology.

I am also thankful to the Oncology Research Department at Park Nicollet Institute and to all those research subjects who participated in our clinical studies. I thank Carol Betz and statisticians, James Hodges and Scott Lunos for helping me with the clinical studies. I would like to thank Dr. Timothy Griffin and Dr. Ebbing de Jong for helping me with proteomics study.

Many friends have helped me through these challenging years. I greatly value their friendship and I deeply appreciate their belief in me.

Finally, and most importantly, none of this would have been possible without the love and support of my family. The personal sacrifices my parents made have allowed me to reach new levels of excellence. I am lucky to have a family that shares 
my enthusiasm for academic pursuits. I would like to express my heart-felt gratitude to my brother, Vijay Thumbigere Math, and to my wife, Amulya Belludi for her patience and unwavering love. I also appreciate the endless love, concern, and support offered by my grandparents and extended family. 


\section{DEDICATION}

This dissertation is dedicated to my parents who taught me how to learn. 


\begin{abstract}
Bisphosphonates (BP), potent osteoclast inhibitors, play a key role in managing patients with osteoporosis, Paget's disease, bone metastasis, and multiple myeloma. BP's anti-resorptive activity substantially reduce fracture risk by $40 \%-70 \%$ in osteoporosis patients, and improve quality of life in cancer patients by preventing skeletal complications. However, prolonged BP use is associated with a significant dental complication termed "Bisphosphonate-Related Osteonecrosis of the Jaw (BRONJ)". To date, the true incidence, etiology, and risk factors that contribute to BRONJ pathogenesis are unknown.
\end{abstract}

In this dissertation, we assessed the frequency, etiology and risk factors that contribute to BRONJ pathogenesis. We noted that the BRONJ frequency in cancer patients is around $3.1 \%$. Factors such as poor periodontal status, diabetes, smoking, prolonged duration of BP therapy, and higher numbers of BP infusions significantly increase the risk of developing BRONJ. In addition, long-term BP administration adversely affects jawbone mechanical properties that could result in increased microdamage accumulation within the jaw bones. Furthermore, using proteomic analysis we identified 200 salivary proteins that were differentially expressed in BRONJ subjects. A majority of the differentially expressed proteins were predicted to have a role in dermatological diseases, genetic disorders, immunological diseases, and inflammatory responses. Finally, analysis of serum samples revealed that VEGF levels are significantly suppressed in patients undergoing BP therapy. In summary, results from this dissertation provide insight into the pathogenesis of BRONJ development. 


\section{TABLE OF CONTENTS}

\section{PAGE}

ACKNOWLEDGEMENTS

i

DEDICATION

iv

ABSTRACT

V

TABLE OF CONTENTS

vi

LIST OF TABLES

$\mathrm{X}$

LIST OF FIGURES

xii

\section{CHAPTER 1 - Introduction and statement of purpose}

Introduction - Bisphosphonates 2

$\begin{array}{lr}\text { Chemical Structure } & 2\end{array}$

History 3

Clinical Application $\quad 4$

Types of Bisphosphonates $\quad 5$

$\begin{array}{ll}\text { Mode of Administration and Bioavailability } & 7\end{array}$

$\begin{array}{ll}\text { Mechanism of Action } & 8\end{array}$

$\begin{array}{ll}\text { Adverse Events } & 13\end{array}$

Bisphosphonate-Related Osteonecrosis of the Jaw (BRONJ) 14

$\begin{array}{ll}\text { Statement of purpose and Significance } & 22\end{array}$

$\begin{array}{ll}\text { Hypothesis } & 24\end{array}$

$\begin{array}{ll}\text { Specific aims } & 24\end{array}$ 
CHAPTER 2 - A Retrospective Study Evaluating Frequency and

Risk factors of Osteonecrosis of the Jaw in 576 Cancer Patients

Receiving Intravenous Bisphosphonates

$\begin{array}{ll}\text { Introduction } & 30\end{array}$

Patients and Methods 31

$\begin{array}{ll}\text { Results } & 34\end{array}$

$\begin{array}{ll}\text { Discussion } & 37\end{array}$

$\begin{array}{ll}\text { Tables } & 43\end{array}$

$\begin{array}{ll}\text { Figures } & 49\end{array}$

CHAPTER 3 - Periodontal Disease and Bisphosphonate-Related $\quad 50$

Osteonecrosis of the Jaw

Introduction $\quad 53$

Patients and Methods $\quad 54$

$\begin{array}{ll}\text { Results } & 56\end{array}$

Discussion $\quad 59$

$\begin{array}{ll}\text { Tables } & 65\end{array}$ 


\section{Osteonecrosis of the Jaw}

Introduction

Materials and Methods

Results

78

Discussion

82

Figures

Tables

88

CHAPTER 5 - Serum Bone Turnover and Angiogenesis Markers

in Bisphosphonate-Related Osteonecrosis of the Jaw

Introduction

Patients and Methods

Results

99

Discussion

Tables 
-Treated Rat Mandible

$\begin{array}{ll}\text { Introduction } & 115\end{array}$

$\begin{array}{ll}\text { Materials and Methods } & 116\end{array}$

$\begin{array}{lr}\text { Results } & 119\end{array}$

$\begin{array}{ll}\text { Discussion } & 121\end{array}$

$\begin{array}{lr}\text { Figures } & 125\end{array}$

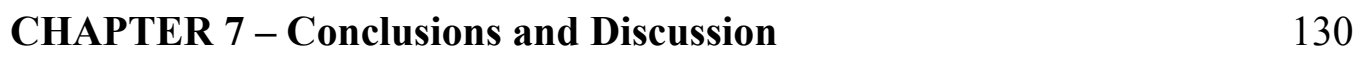

Chapter 8 - Supplementary Data - Clinical features, risk factors, 143

Management and treatment outcomes of 26 BRONJ patients

$\begin{array}{ll}\text { Introduction } & 146\end{array}$

$\begin{array}{ll}\text { Patients and Methods } & 147\end{array}$

$\begin{array}{ll}\text { Results } & 150\end{array}$

$\begin{array}{ll}\text { Discussion } & 153\end{array}$

$\begin{array}{lr}\text { Tables } & 158\end{array}$

$\begin{array}{lr}\text { Figures } & 162\end{array}$

$\begin{array}{ll}\text { References } & 166\end{array}$ 


\section{LIST OF TABLES}

\section{CHAPTER 1}

Table 1. Bisphosphonate Formulations $\quad 5$

Table 2. Structure and Relative Potency of Bisphosphonates 6

\section{CHAPTER 2}

Table 1. Patient Characteristics 43

Table 2. Bisphosphonate Treatment 45

Table 3. BRONJ Characteristics 46

Table 4. Risk Factors for Developing BRONJ 47

Table 5. Risk Factors for Time to Development of BRONJ 48

\section{CHAPTER 3}

Table 1. Baseline Patient Characteristics 65

Table 2. Unadjusted Comparisons of Clinical Periodontal Measurements 66

Table 3. Adjusted Comparisons $\S$ of Clinical Periodontal Measurements $\quad 67$

$\begin{array}{lc}\text { Table 4. BRONJ Characteristics } & 68\end{array}$

Table 5. Unadjusted Comparisons of Radiographic Alveolar Bone Height 69

Table 6. Adjusted Comparisons of Radiographic Alveolar Bone Height $\quad 70$

\section{CHAPTER 4}

$\begin{array}{lr}\text { Table 1. Baseline Patient Characteristics } & 88\end{array}$

Table 2. Differentially expressed salivary proteins among BRONJ cases $\quad 89$ in both the high BP infusion and low BP infusion groups 
Table 3. Differentially expressed salivary proteins in low BP infusion

BRONJ group compared to high BP infusion control group

\section{CHAPTER 5}

$\begin{array}{lr}\text { Table 1. Baseline Patient Characteristics } & 110\end{array}$

$\begin{array}{ll}\text { Table 2. Serum Biochemical Markers } & 111\end{array}$

\section{Chapter 8 - Supplementary Data}

$\begin{array}{ll}\text { Table 1. Characteristics of BRONJ Patients } & 158\end{array}$

$\begin{array}{lr}\text { Table 2. BRONJ Characteristics } & 160\end{array}$

$\begin{array}{ll}\text { Table 3. BRONJ Management outcome } & 161\end{array}$ 


\section{LIST OF FIGURES}

\section{CHAPTER 1}

Figure 1 The structure of germinal bisphosphonate compared with pyrophosphate

Figure 2 Mechanism of action of bisphosphonates on osteoclasts

Figure 3 Clinical presentation of BRONJ

\section{CHAPTER 2}

Figure 1 Proportion of patients without BRONJ over time

according to type of bisphosphonate therapy received

\section{Chapter 4}

Figure 1 Biomarker Discovery Method

\section{Chapter 6}

Figure 1 Microhardness results of trabecular and cortical bones

Figure 2 Microhardness results of Mandible and Femur

Figure 3 Microhardness results of Anterior and Posterior

region of the Mandible

Figure 4 SEM image showing higher osteon density

Figure 5 Histological image showing bone density

\section{Chapter 8 - Supplementary Data}

$\begin{array}{lll}\text { Figure } 1 \text { Management of advanced BRONJ lesion } & 162\end{array}$

$\begin{array}{lll}\text { Figure } 2 \text { Resolution of Spontaneous BRONJ lesion } & 164\end{array}$

$\begin{array}{lll}\text { Figure } 3 & \text { Histological image of BRONJ lesion } & 165\end{array}$ 
CHAPTER 1

INTRODUCTION

AND STATEMENT OF PURPOSE 


\section{INTRODUCTION}

\section{BISPHOSPHONATES}

\section{Chemical Structure}

BPs are pyrophosphate analogs with high affinity for hydroxyapatite (calcium phosphate) crystals. $[1,2,3]$ They have a chemical structure similar to that of inorganic pyrophosphate.[3] Pyrophosphate is comprised of two phosphate groups linked by phosphoanhydride bonds (P-O-P structure), whereas, BP contains two phosphate groups linked by phosphoether bonds to a central germinal carbon atom (P-C-P structure) (Figure 1).
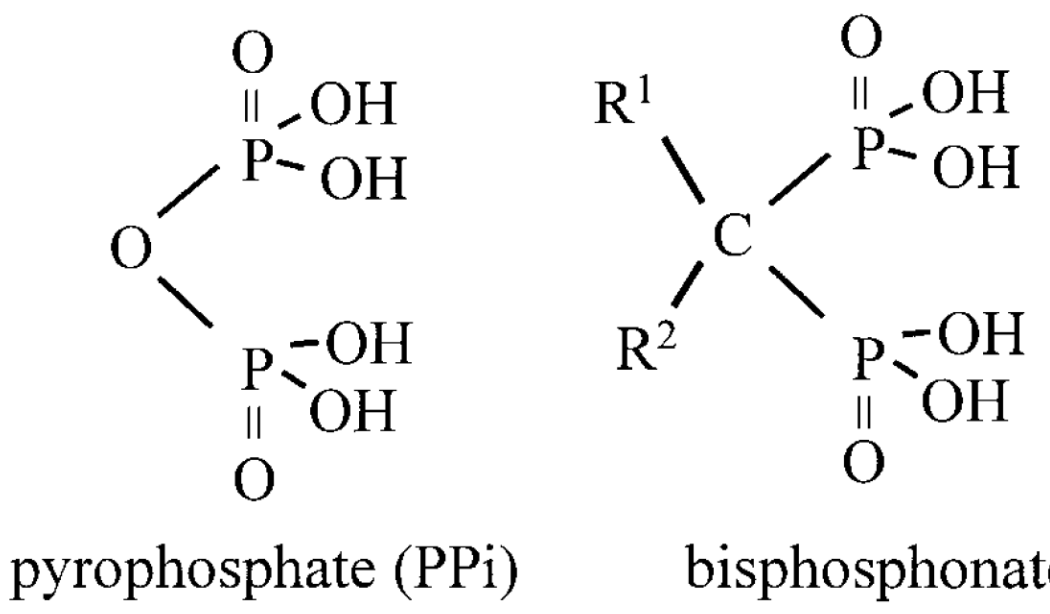

bisphosphonate

Figure 1. The structure of germinal bisphosphonate compared with pyrophosphate

(Adapted from Rogers MJ, Gordon S, Benford HL, et al. Cellular and molecular mechanisms of action of bisphosphonates)[4] 
Unlike the P-O-P bonds of pyrophosphate, the P-C-P structure of BPs are stable to heat and most chemical reagents, and highly resistant to hydrolysis under acidic conditions. The two covalently bonded groups (side chains) attached to the geminal carbon atom are referred to as $R_{1}$ and $R_{2}$ chains (Figure 1). By having one of the side chains $\left(\mathrm{R}_{1}\right)$ as a hydroxyl $(-\mathrm{OH})$ or primary amino group $\left(-\mathrm{NH}_{2}\right)$, BPs affinity for calcium can be further increased. This allows the formation of a tridentate conformation that is able to bind $\mathrm{Ca}^{2+}$ more effectively.[1] The high affinity of BP for $\mathrm{Ca}^{2+}$ ions is the basis of the bone-targeting property of these compounds.

\section{History}

BPs were first developed in the 1800 s for non medicinal use. They were mainly used as corrosion inhibitors or as complexing agents in the textile, fertilizer, and oil industry. Similar to pyrophosphates, BPs were used as "water softeners" because of their ability to inhibit calcium phosphate precipitation. A key step towards the clinical use of BP occurred when it was discovered that BP could also inhibit the dissolution of hydroxyapatite crystals.[5,6,7] Only in the last 40 years they have been developed as drugs for clinical use in treating various diseases of bone, tooth, and calcium metabolism. Initially, they were tested in Paget's disease of bone, then in hypercalcemia of malignancy, multiple myeloma, and bone metastasis, and much later in osteoporosis. Etidronate was the first BP approved in the United States in 1977 followed by pamidronate in 1991, alendronate in 1995, risedronate in 1998, zoledronate in 2001, and ibandronate in 2005. 


\section{Clinical Application}

Osteoporosis, a major health problem, affects nearly 10 million Americans over the age of 50 years and is the most common cause of fractures in older adults.[8] More than two million fractures occur each year as a result of osteoporosis or osteopenia. Osteoporotic hip fractures are associated with the highest morbidity and mortality. Currently, bisphosphonate (BP) are the first-line therapy for prevention of fractures in osteoporosis patients[9,10,11,12] with over 17 million prescriptions sold per year.[13] Anti-resorptive activity of BP substantially reduces fracture risk by $40 \%-70 \%$ in osteoporosis patients. $[9,14,15]$

Similarly, BP have become the standard of care for patients with malignant bone disease. Bone metastases are associated with considerable skeletal morbidity, including severe bone pain, pathologic fractures, spinal cord compression, and hypercalcemia of malignancy. These skeletal complications occur as a result of excessive bone metabolism, primarily bone resorption. By inhibiting bone resorption, BP significantly reduce the incidence of skeletal complications and prevent bone metastasis in cancer patients. In addition, BP are reported to have direct antiproliferative and proapoptotic effects on cancer cells which can further reduce the incidence of bone metastases.[16,17,18]

BP have also proven beneficial in other metabolic bone disorders like Paget's disease and congenital pathologies such as osteogenesis imperfecta.[19] 


\section{Types of BP}

Several years after the initial discovery that BP could inhibit bone resorption, a new class of BP was identified that were up to 10,000-fold more potent at inhibiting bone resorption. It was found that insertion of a nitrogen atom at critical positions in the side chain of the molecule significantly increased the potency level. Based on the nitrogen atom, BP are currently divided into non-nitrogen containing BP (clodronate, etidronate, tiludronate) and nitrogen-containing BP (pamidronate, alendronate, risedronate, ibandronate and zoledronate). Table 1 illustrates different types of nonnitrogen containing and nitrogen-containing BP approved by the Food and Drug Administration (FDA).

\begin{tabular}{|c|c|c|c|c|c|}
\hline Generic Name & Brand Name & Manufacturer and Location & Dosage Forms & $\begin{array}{l}\text { Nitrogen- } \\
\text { Containing }\end{array}$ & $\begin{array}{l}\text { FDA Approval } \\
\text { Date }\end{array}$ \\
\hline Etidronate disodium & Didronel & $\begin{array}{l}\text { Procter \& Gamble Pharmaceuticals, } \\
\text { Cincinnati, Ohio }\end{array}$ & 200- and $400-\mathrm{mg}$ tablets & No & $\begin{array}{l}1 \text { September } \\
1977\end{array}$ \\
\hline Clodronate disodium & Bonefos (Canada) & Schering AG, Berlin, Germany & $\begin{array}{l}400-\text { and } 800-\mathrm{mg} \text { tablets; } 60 \mathrm{mg} / \mathrm{mL} \\
\text { ampulet }\end{array}$ & No & Not approved \\
\hline Tiludronate disodium & Skelid & $\begin{array}{l}\text { Sanofi-Synthelabo Inc., New York, } \\
\text { New York }\end{array}$ & 200-mg tablet & $\begin{array}{l}\text { No; sulfur } \\
\text { moiety }\end{array}$ & 7 March 1997 \\
\hline Alendronate sodium & Fosamax & $\begin{array}{l}\text { Merck \& Co. Inc., Whitehouse } \\
\text { Station, New Jersey }\end{array}$ & $\begin{array}{l}5-, 10-, 35-, 40-, \text { and } 70-\mathrm{mg} \text { tablets; } \\
70 \mathrm{mg} / 75 \mathrm{~mL} \text { oral solution }\end{array}$ & Yes & $\begin{array}{l}29 \text { September } \\
1995\end{array}$ \\
\hline $\begin{array}{l}\text { Alendronate sodium } \\
\text { plus vitamin } D_{3}\end{array}$ & Fosamax plus $D$ & $\begin{array}{l}\text { Merck \& Co. Inc., Whitehouse } \\
\text { Station, New Jersey }\end{array}$ & $\begin{array}{l}\text { 70-mg and } 2800-U \text { cholecalciferol } \\
\text { tablet }\end{array}$ & Yes & 7 April 2005 \\
\hline Pamidronate disodium & Aredia & $\begin{array}{l}\text { Novartis Pharmaceuticals, East } \\
\text { Hanover, New Jersey }\end{array}$ & $30-, 60-$, and $90-\mathrm{mg}$ vialst & Yes & 31 October 1991 \\
\hline Risedronate sodium & Actonel & $\begin{array}{l}\text { Procter \& Gamble Pharmaceuticals, } \\
\text { Cincinnati, Ohio }\end{array}$ & 5-, 30-, and 35-mg tablets & Yes & 27 March 1998 \\
\hline $\begin{array}{l}\text { Risedronate sodium } \\
\text { plus calcium }\end{array}$ & Actonel with calcium & $\begin{array}{l}\text { Procter \& Gamble Pharmaceuticals, } \\
\text { Cincinnati, Ohio }\end{array}$ & 35-mg and 500-mg calcium tablets & Yes & 12 August 2005 \\
\hline Zoledronic acid & Zometa & $\begin{array}{l}\text { Novartis Pharmaceuticals, East } \\
\text { Hanover, New Jersey }\end{array}$ & 4-mg vialt & Yes & 20 August 2001 \\
\hline Ibandronate sodium & Boniva & $\begin{array}{l}\text { Roche Laboratories Inc., Nutley, } \\
\text { New Jersey }\end{array}$ & $\begin{array}{l}2.5-\mathrm{mg} \text { tablet } \\
150-\mathrm{mg} \text { tablet } \\
3 \mathrm{mg} / 3 \mathrm{mLt}\end{array}$ & Yes & $\begin{array}{l}16 \text { May } 2003 \\
24 \text { March } 2005 \\
6 \text { January } 2006\end{array}$ \\
\hline
\end{tabular}

(Table 1. Adapted from Woo SB, Hellstein JW, Kalmar JR. Narrative [corrected] review: bisphosphonates and osteonecrosis of the jaws) [20] 
Table 2. Structure and relative potency of bisphosphonates

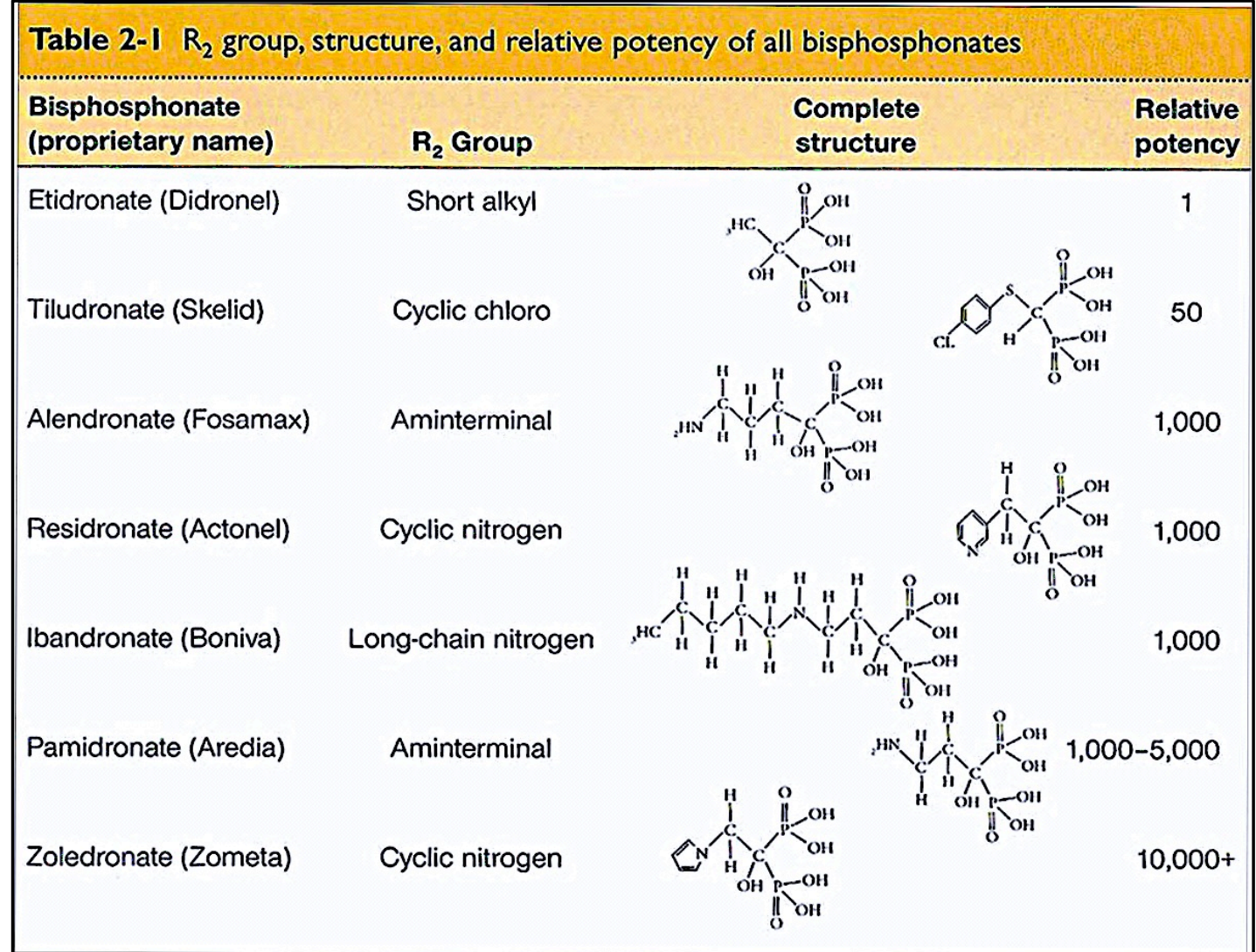

(Table 2. Adapted from Marx RE: Oral \& Intravenous Bisphosphonate-induced Osteonecrosis of the Jaws: History, Etiology, Prevention and Treatment. Quintessence Publishing Co Inc., U.S.) 


\section{Mode of Administration and Bioavailability}

Based on the mode of administration, BP are further classified into oral (etidronate, tiludronate alendronate, risedronate, ibandronate) and intravenous BP (pamidronate, zoledronate). For oral administration, patients are required to fast several hours before drug ingestion and to remain upright and avoid eating food for at least 30 minutes after drug ingestion. Taking BP with food or anything containing divalent cations will completely block its absorption. Therefore, BP are never given at mealtimes and never together with milk, dairy products or with iron supplements. For some unknown reasons, orange juice and coffee also decrease absorption. Under ideal conditions $<1 \%$ of the orally administered dose is absorbed. About fifty percent of the absorbed dose binds to the bone surface, mostly at sites of active bone remodeling. The skeletal uptake varies with age, sex and with the dose and nature of the compound.

Although bioavailability of oral BP is very low, adequate levels to inhibit bone resorption can be reached within a few weeks.[21] However, the amount absorbed is inadequate to inhibit a strong pro-resorptive stimulus that occurs in metastatic bone disease and multiple myeloma. Therefore, for these latter conditions, intravenous infusions of nitrogen-containing BP such as pamidronate and zoledronate are preferred.[22,23] Intravenous infusions of BP produce rapid anti-resorptive action within 24-48 hours of infusion. Oral BP are mainly used to treat osteoporosis and Paget's disease. $[9,12]$ 
Regardless of the mode of administration, BP are rapidly cleared through the kidneys and into bone so that exposure in soft tissues, such as the bone marrow, is transient. Urinary excretion is decreased in renal failure and the removal by peritoneal dialysis is poor, which has to be accounted for when BP are administered in patients with kidney disease. Sometimes BP, especially pamidronate, can deposit in other organs, mostly the liver and the spleen. The deposition is proportionally greater when large amounts of the compounds are given. BP are also bioavailable to some extent when given intra-nasally and through the skin. This may open new modes of administration in the future.

\section{Mechanism of action}

Osteoclasts, the multinucleated bone resorbing cells, are the ultimate target of BP action. Due to their high affinity for hydroxyapatite crystals, BP are directed to areas of bone turnover and are especially concentrated at sites of osteoclastic bone resorption.[24,25] Once bound to hydroxyapatite crystals, minimal amounts of BP are released into the circulation.[26] During bone resorption, BP are released from hydroxyapatite crystals and internalized by osteoclasts.[27] Following internalization, BP reduce osteoclastic bone resorption directly through 4 mechanisms: (a) inhibition of osteoclast recruitment to the bone surface[28]; (b) inhibition of osteoclast adhesion[29]; (c) inhibition of osteoclast activity on the bone surface[27,30]; and (d) shortening of the osteoclast life span by promoting its apoptosis.[31,32] Furthermore, 
BP alter angiogenesis[33,34] and signal transduction between osteoclasts and osteoblasts.[35]

BP can have direct effects on osteoclasts or its precursors, or indirectly exert action on cells that modulate the osteoclasts. BP action on mature osteoclasts causes subtle changes in osteoclast structure that affect the osteoclast ability to resorb bone. A characteristic feature is the loss of osteoclast ruffled border[36,37,38] (the convoluted region of plasma membrane adjacent to the bone surface that is essential for the resorption process). Also, BP can disrupt the osteoclast cytoskeleton and cause loss of actin rings $[24,36,39,40]$, which are required for polarization and the formation of a sealing zone at the bone surface. Furthermore, inhibition of cellular metabolism could indirectly affect processes required for resorption such as release of lysozomal enzymes[41] or acidification brought about by the activity of the ATP-dependent proton pump in the ruffled border.[42]

Previous studies suggest that some BP may also inhibit osteoclast precursors, thereby preventing osteoclast formation.[28,43] Boonekamp et al noted that low concentrations of pamidronate prevented the recruitment, differentiation, and fusion of osteoclasts precursors.[28] The order of BP potency for inhibiting osteoclast formation is as follows: risedronate $>$ pamidronate $>$ neridronate $>$ clodronate >etidronate.[44] Furthermore, there have been reports that BP inhibit the proliferation of osteoblast-like cells and other connective tissue cells (calvarial cells, fibroblasts, and chondrocytes).[45,46] By inhibiting the secretion of osteoclast-stimulating factor 
or by stimulating the production of osteoclast-inhibitory factors from osteoblasts, BP can indirectly regulate the activity of osteoclasts. Sahni et al. found that treatment of osteoblast-like CRP 10/30 cells (which are potent stimulators of osteoclast activity) with $10^{-7} \mathrm{M}$ ibandronate or $10^{-6} \mathrm{M}$ clodronate inhibited bone resorption when these cells were subsequently co-cultured with osteoclasts.[47]

The non-nitrogen containing BP inhibit bone resorption primarily by inducing osteoclast apoptosis[48]. In contrast, the more potent nitrogen-containing BP function by inhibiting farnesyl diphosphate (FPP) synthase, a key enzyme of the mevalonate pathway and the downstream metabolites essential for osteoclast function. [3,26,49] Mevalonate pathway is responsible for the production of cholesterol and isoprenoid lipids such as isopentenyl diphosphate (IPP), farnesyl diphosphate (FPP), and geranylgeranyl diphosphate (GGPP) (Figure 2).[50,51]

Inhibition of FPP synthase by nitrogen-containing BP leads to decreased formation of FPP and GGPP.[50,51] FPP and GGPP are required for posttranslational prenylation (transfer of fatty acid chains) of proteins, including GTP binding proteins such as Ras, Rab, Rho and Rac. [50,51,52] These proteins are important for osteoclast function including cytoskeletal assembly, membrane ruffling and intracellular signaling.[53,54,55] Disruption of GTP binding proteins leads to decreased osteoclast activity and increased apoptosis.[56] 
Figure 2. Mechanism of action of bisphosphonates on osteoclasts

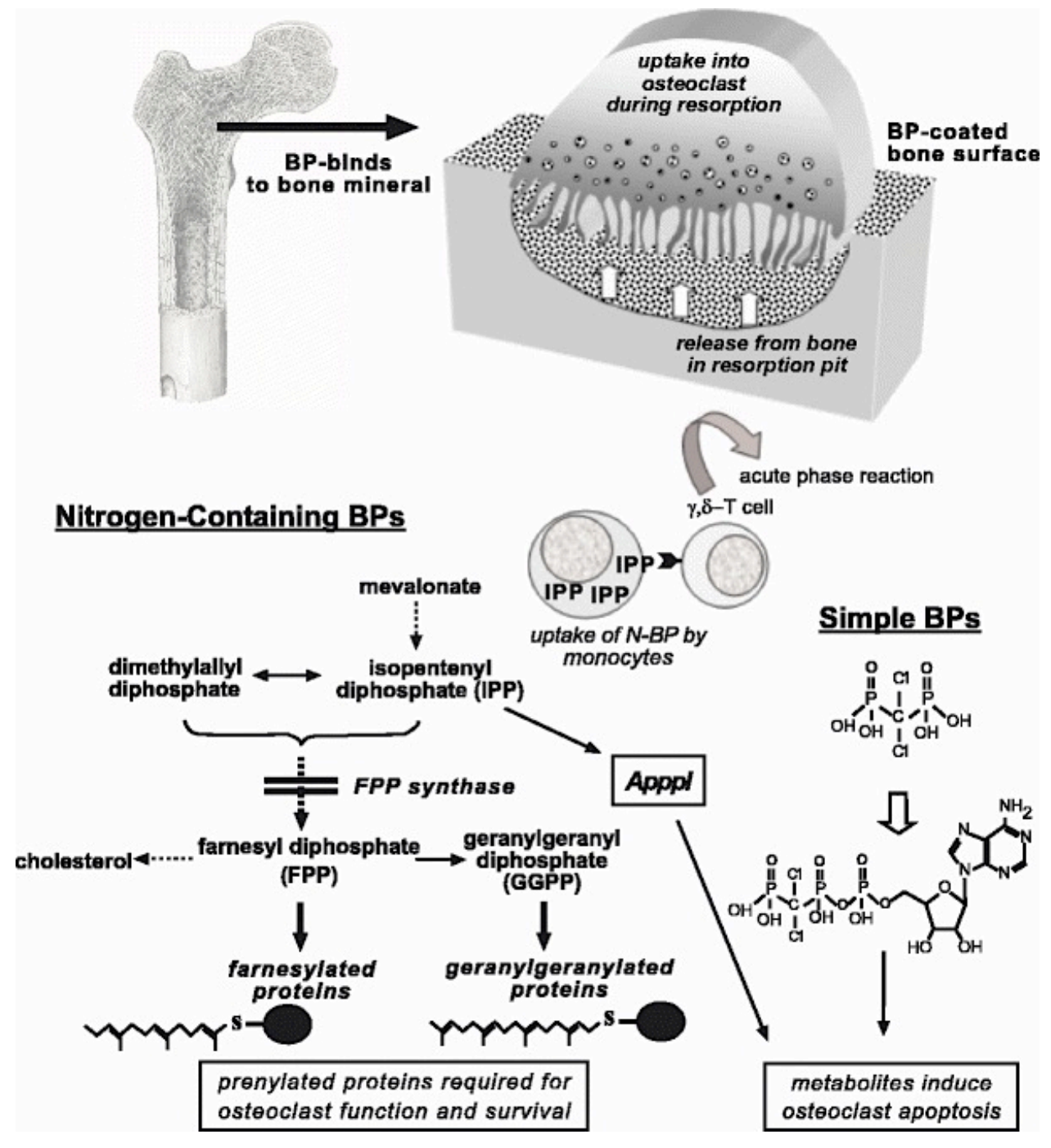

After binding to bone mineral, BP are internalized into osteoclasts by endocytosis. Simple BP (e.g., clodronate, etidronate) are metabolized in the osteoclast cytosol to ATP analogues that induce osteoclast apoptosis. Nitrogen-containing BP inhibit FPP synthase, thereby preventing the prenylation of small GTPase proteins essential for the function and survival of osteoclasts. Inhibition of FPP synthase also causes the accumulation of IPP, which is incorporated into ApppI (an analogue of ATP capable of inducing osteoclast apoptosis).

(Figure 2. Adapted from Russell RG et al. Mechanisms of action of bisphosphonates: similarities and differences and their potential influence on clinical efficacy)[57] 
BP alternative pharmacological action inhibits angiogenesis. Treatment with pamidronate or zoledronate has been reported to reduce vascular endothelial growth factor (VEGF) levels.[58,59] In a subcutaneous murine growth factor implant model, zoledronic acid strongly inhibited the angiogenic response induced by basic fibroblast growth factor (bFGF) and vascular endothelial growth factor (VEGF)[60]. In humans, Santini et al. showed a significant decrease of circulating VEGF levels in patients with advanced solid cancer and bone metastases receiving a single dose of pamidronate administration[58]. Kapitola et al noted that pamidronate reduced bone blood flow in the rat femur and tibia, and associated this with an inhibition of ${ }^{45} \mathrm{Ca}$ and ${ }^{3} \mathrm{H}$-proline incorporation into bone.[61]

Experimental evidence suggests that BP also inhibits capillary neoangiogenesis. Fournier et al. demonstrated that BP decrease capillary tube formation and vessel sprouting.[33] Wood et al. showed that BP produced a dosedependent inhibition of endothelial proliferation in cultured human umbilical vein and rat aortic ring cells.[62] The same investigators have demonstrated that zoledronic acid is able to reduce vessel sprouting in the chicken egg chorioallantoic membrane assay.[62]

It is now well know that osteoclasts are important for bone angiogenesis.[63] BP anti-osteoclastic activities might also indirectly diminish skeletal vasculature. Shoutens et al. observed an increase in the number of osteoclasts with a concomitant 
increase in bone blood flow, whereas suppression of bone resorption was associated with decreased blood flow.[64]

\section{Adverse Events}

BP are generally well-tolerated. However, high doses, prolonged use, and impaired elimination of these drugs are associated with adverse events including bone pain, osteomalacia and serious complications like gastric ulcers, nephrotoxicity and Bisphosphonate-Related Osteonecrosis of the Jaw (BRONJ).[65,66,67]

A number of adverse gastrointestinal events have been associated with oral administration of BP including gastrointestinal erosions, ulcers, perforations and strictures. The incidence of these adverse events is dose and dose-frequency dependent. They probably occur when high local concentrations of BP cause toxic levels in adjacent cells as the tablets dissolve, but they can also occur at the standard osteoporosis doses. BP treatment is sometimes associated with bone pain, particularly when administered intravenously. Bone pain is generally considered part of an acute phase reaction secondary to release of inflammatory cytokines such as interleukin-6 and tumor necrosis factor- $\alpha .[65]$ Acute tubular necrosis has been reported in patients receiving high doses of intravenous pamidronate or zoledronate.[68,69] Renal damage can occur if BP are delivered too rapidly by intravenous infusions, as BP are rapidly cleared in the kidney. Although irreversible damage has been reported, renal function usually returns to normal once the drug is discontinued.[70] Recently, some 
epidemiological studies have suggested an association between BP and atrial fibrillation.[71,72,73] A plausible mechanism includes electrolyte imbalance such as hypocalcemia or BP-induced inflammation affecting atrial remodeling and fibrosis.[74,75,76] However, the toxicity that has received widespread attention and has raised the most concern in recent years is BRONJ.

\section{Bisphosphonate-Related Osteonecrosis of the Jaw (BRONJ)}

The risk of osteonecrosis associated with phosphorous compounds was first described in the $19^{\text {th }}$ century.[77] An occupational disease termed "phossy jaw" or "phosphorus necrosis of the jaw" was noted among workers in the matchmaking industry who worked with white phosphorus.[78] Workers presented with pain, infection, and exposure of the jaw bone. The incidence of "phossy jaw" started decreasing over the years with reduced use of white phosphorous in industry and improved working conditions. However, in 1999 reports of ulceration of the oral mucosa as a complication of oral BP therapy started emerging.[79] It was thought the ulceration were a result of direct mucosal injury, similar to esophageal ulceration that is another recognized side effect of alendronate therapy.[80] In fact, BRONJ was never reported in any of the initial clinical trials. By 2001, oral and intravenous BP therapy had been in widespread use and the incidence of BRONJ had increased. Consequently, cases were reported at scientific meetings and published in the literature in 2003.[81,82,83] Since then, over 1000 cases of BRONJ have been reported in the literature.[20,84] 
The American Association of Oral and Maxillofacial Surgeons (AAOMS) defines BRONJ as "exposed bone in the maxillofacial area occurring in the absence of head and neck irradiation and showing no evidence of healing for at least 8 weeks after identification in patients treated with BP therapy". [85]

To date, the true incidence of BRONJ is unknown. An overwhelming proportion (97\%) of the reported cases are related to high-dose intravenous BP used in cancer patients $[84,86,87,88]$, although a small fraction $(3 \%)$ has been reported in osteoporosis and Paget's disease patients receiving oral BP. [89,90,91] Furthermore, BRONJ frequency appears to be higher in multiple myeloma $(55.9 \%)$ and breast cancer (33.4\%) patients.[84] A plausible explanation for increased risk in cancer patients is that the severity of cancer, chemotherapy and corticosteroids may modulate immunological status, increasing the risk of infection and impairing wound healing. Also, cancer patients receive up to 12-50 times higher doses of BP than osteoporosis patients.

The estimated incidence of BRONJ in osteoporosis patients receiving oral BP is around 0.7 per 100,000 person/years of exposure. [89,92,93] The incidence of BRONJ in cancer patients receiving intravenous BP range from $0.72 \%$ to 7.4\%.[86,94,95,96,97] The wide disparity in incidence rate among published studies are attributable to variations in BP treatment regimen (type, dose, duration), nature of 
study (single center studies evaluating subset of cancer population), age, status of disease, and cancer management.

BRONJ is defined as an intraoral complication of BP therapy. It is suggested that structural anatomy and physiology of the jaw, micro-organism rich environment of the oral cavity and physiologic stresses of mastication could be significant cofactors in the development of BRONJ. However, recently, cases of atypical femoral fractures and osteonecrosis of the external auditory canal associated with BP therapy have been reported in the literature.[98,99,100,101]

BRONJ lesions may be asymptomatic or present with pain, purulent discharge, swelling, tooth mobility and paresthesia, culminating with reduced ability to eat and speak.[102,103] BRONJ occurs more frequently $(65 \%)$ in the mandible, followed by $26 \%$ in the maxilla and $9 \%$ in both jaws.[20] BRONJ frequency is higher in the posterior region of the jaw[20,102]. Around $60 \%$ of the BRONJ lesions occur after dental procedure, while $40 \%$ of them are spontaneous.[20,84] Radiographic evaluation is usually inconclusive for early lesions, whereas in advanced cases, a poorly defined moth-eaten radiolucency is noted with radio-opaque sequestra.

Currently, there is no effective treatment for BRONJ and discontinuing BP is not helpful as these compounds tend to reside in the bone for long periods.[26,104] Radical surgical intervention often exacerbates the condition and the role of hyperbaric oxygen treatment in BRONJ management has not been completely 
understood. Patients are usually treated conservatively with minimal debridement, antibiotics and chlorhexidine mouth rinse to limit the extent of the damage.

Figure 3: Clinical presentation of BRONJ
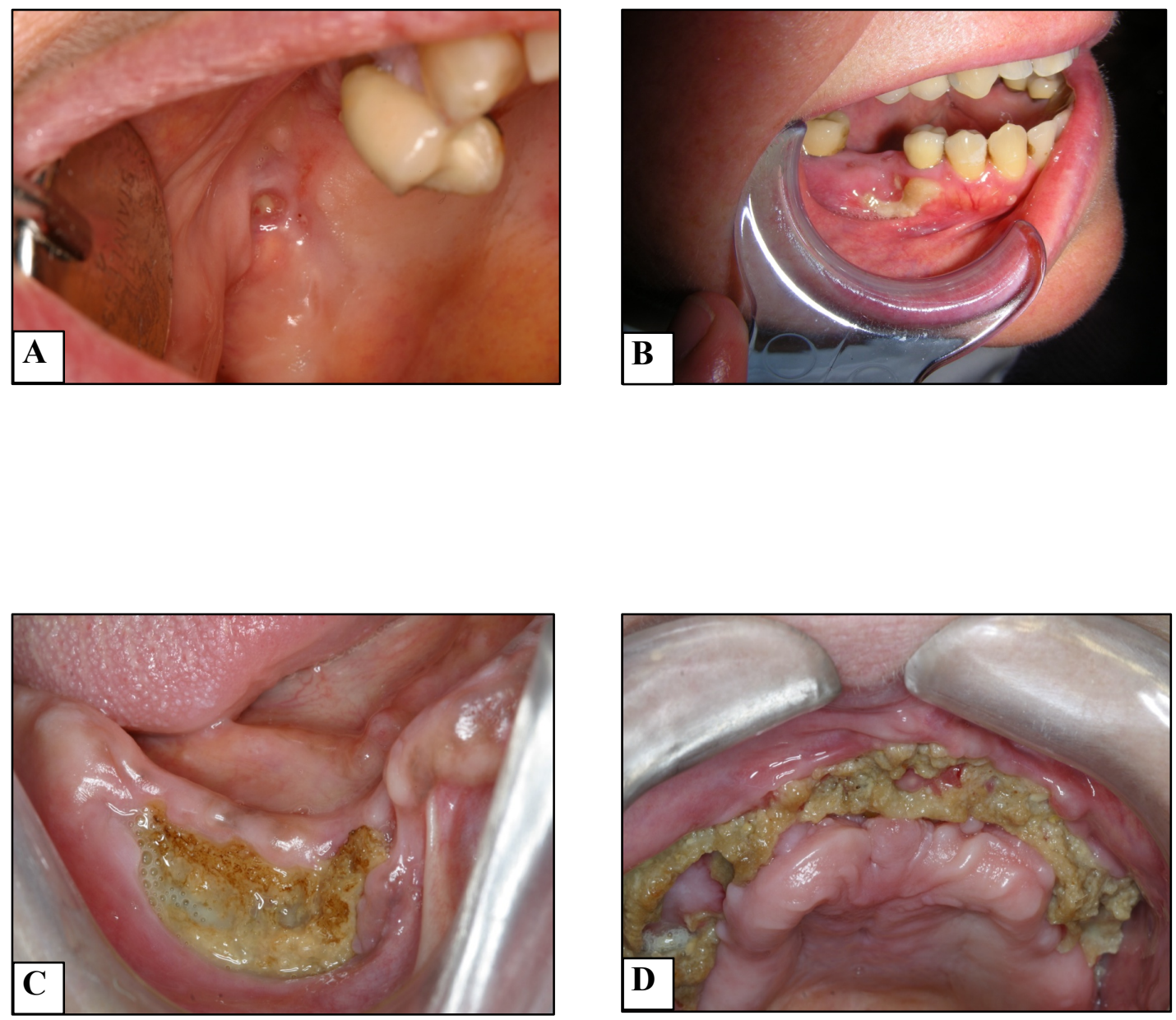

BRONJ lesions may be small and symptomatic (a) or could be advanced involving the entire segment of the jaw (D). 


\section{Potential risk factors for BRONJ development}

A review of published literature suggests a multifactorial contribution to the development of BRONJ, with certain factors being associated more often than others. Factors such as trauma from dental surgery, advanced cancer, chemotherapy, corticosteroids, dental infection, diabetes, tobacco and alcohol use seem to increase the risk of BRONJ development. [20,84,85,86,91,102,103,105]

\section{Dental Trauma:}

Trauma from dental procedures such as tooth extraction and periodontal surgery has been most frequently associated with BRONJ development. Woo et al. summarized that $60 \%$ of reported BRONJ cases have been associated with some form of dentoalveolar surgery.[20] Ruggeiro et al. in their study noted that $86 \%(54 / 63)$ of the patients had a history of dentoalveolar surgery.[103] Comparable findings have also been noted in other large clinical studies.[102,106] In fact, Badros et al. noted that a patient is 9 times more likely to develop BRONJ following tooth extraction.[106] Marx et al. reported that BRONJ cases were also related to other dental procedures such as such as periodontal surgery (11.2\%), dental implant placement (3.4\%) and surgical endodontic procedures $(0.8 \%)$.[102] A recent publication also reported BRONJ development in association with non-surgical root canal treatment.[107] Woo et al. reported that the $40 \%$ of BRONJ cases that occur spontaneously are probably related to infection, denture trauma or other physical trauma.[20] BRONJ lesions most frequently occur in the posterior lingual region of the mandible and are similar to lingual mandibular sequestration (which occurs in healthy adults). The etiology of 
lingual mandibular sequestration is thought to be related to minor trauma that causes local damage to the thin mucosa and underlying periosteum, leading to bone necrosis.[108,109] These lesions are known to spontaneously resolve. Other locations of spontaneous BRONJ development include sites that can be easily traumatized such as exostoses or palatal tori.[102] Furthermore, a strong association has been established between ill-fitting dentures and BRONJ development.[84] However, all of these findings are difficult to interpret due to lack of information regarding prevalence of various types of trauma in the BRONJ lesion site, and in matched controls.

\section{Periodontal Disease:}

Marx et al. noted that $84 \%$ of their BRONJ cases had periodontal disease, $29 \%$ with advanced periodontitis.[102] Ficarra et al. reported 9 cases of BRONJ following extraction of periodontally "hopeless" teeth.[110] An association between periodontal disease and BRONJ has also been suggested in various other publications; however, no detailed information about periodontal disease in BRONJ cases has been reported.[20,84,106,111] A study determining prevalence of periodontal disease in BRONJ patients has not yet been performed. This is particularly significant because almost all BRONJ patients reported in the literature are older and immunosuppressed, which are risk indicators for periodontal disease. Badros et al. isolated putative periodontal pathogens (Prevotella and Porphyromonas species) from BRONJ lesions in 9 out of 17 patients.[106] Lesions in the remaining 8 patients contained Actinomyces species. Others have also isolated Actinomyces species from BRONJ 
lesions.[112,113] The contribution of these organisms to BRONJ development is unknown, and it is vital to determine whether bacterial colonization contributes to BRONJ development, or represents secondary infection. Sedghizadeh et al. showed that bone sections from BRONJ sites contained microbial biofilms comprised predominantly of bacteria such as Fusobacterium, Actinomyces, Staphylococcus, Streptococcus, Selenomonas and Trepenemes.[114]

\section{Other Co-morbidities and Potential Risk Factors:}

As cancer patients represent the major population at risk for developing BRONJ, adjuvant chemotherapy should be considered a major risk factor. Chemotherapeutic agents can modulate immune status of patients thereby increasing the risk for infections and reducing wound healing. In fact, long before BRONJ was recognized, Schwartz et al. reported 2 cases of osteonecrosis of the jaws in patients receiving cancer chemotherapy.[115] The majority of cancer patients also receive corticosteroids as part of their cancer treatment. Corticosteroid effects on osteoblasts have been extensively studied, and they therefore could be potential risk factor for BRONJ development.[116] Furthermore, avascular necrosis of the femoral head is a well-recognized clinical entity in patients on long-term corticosteroid therapy.[117] Other medications such as thalidomide given to multiple myeloma patients should also be considered as potential risk factors due to their inhibitory effects on osteoclast precursor cells.[118] Khamiasi et al. recently reported a strong association between diabetes mellitus and ONJ.[119] The authors noted that $58 \%$ of BRONJ patients in their series either had diabetes mellitus or impaired fasting glucose, which was higher 
compared to diabetes mellitus in the general population (14\%) and compared to cancer patients taking BPs but not having a diagnosis of BRONJ (12\%). 


\section{STATEMENT OF PURPOSE AND SIGNIFICANCE}

At present, there is no predictable cure for BRONJ and discontinuing BP therapy is not helpful as BP's have extended half-life. Advanced cases with extensive necrosis and pathologic fracture often require surgical resection of a significant portion of the jaw bone. This further worsens the morbid situations (cancer, severe osteoporosis) patients are already coping with. To date, no studies have directly investigated BP's causative role in BRONJ development. Most of the information obtained thus far has been through case reports that pose the following limitations towards completely understanding this condition.

1. Although case reports are an important source of information in uncommon events $(<10 \%)$ such as BRONJ, they are subjected to bias in reporting. Voluntary reporting often underestimates the true incidence due to the possibility of undiagnosed cases. Often an unknown number of patients receiving $\mathrm{BP}$ and an unknown number of patients affected by BRONJ have limited the estimation of true incidence.

2. In rare conditions, case reports might provide good insight into the possible risk factors. However, the etiological complexity with lack of a single defined etiologic factor (genetic or environmental), makes it essential to investigate the cause of BRONJ broadly in all etiological categories by undertaking casecontrol and prospective clinical studies. 
The continued increase in BRONJ incidence emphasizes the urgent need to characterize the condition and identify predisposing factors. Although prospective studies would be ideal towards documenting the true incidence and risk factors for BRONJ, the estimated prevalence of BRONJ $(0.72 \%$ to $7.4 \%)$ is very low posing a major roadblock in initiating such studies at this point in time $[84,86,97]$. As an initial step, the projected specific aims in this proposal will help us better understand this rare event and provide the foundation required to initiate future intervention studies. 


\section{HYPOTHESIS}

We hypothesize that long-term BP therapy, periodontal disease, and altered bone biomechanical properties contribute to BRONJ development.

\section{SPECIFIC AIMS}

\section{Specific Aim 1:}

Specific aim 1a: To determine the incidence, risk factors, and clinical presentation of BRONJ

Specific aim 1b: To estimate the cumulative hazard of developing BRONJ among different cancer patients receiving different types of $B P$

Hypothesis 1 (project 1): BRONJ is a complex and multifactorial process related to long-term BP therapy.

Rationale: Previously published case reports have noted an increased incidence of BRONJ in patients receiving BP therapy for longer periods. Also, studies have noted a frequent history of dental infection, diabetes, and corticosteroid therapy among BRONJ subjects. These findings suggest that BRONJ is a complex and multifactorial process related to long-term BP therapy. In order to determine the incidence, risk factors, and clinical presentation of BRONJ we will undertake a retrospective chart review study. 


\section{Specific Aim 2:}

Specific aim 2a: To determine the association between clinical, radiographic, and microbiological measures of periodontitis and risk of developing BRONJ

Specific aim 2b: To determine if BRONJ is associated with increased mucosal invasion of putative periodontal pathogens and Actinomyces species

Hypothesis 2 (project 2): Periodontal disease and periodontal pathogens are risk factors for BRONJ development.

Rationale: Rationale for this hypothesis is based on the confinement of BRONJ lesions to microorganism- rich oral cavity. Our preliminary results demonstrate BRONJ biopsy specimens to be infected with Actinomyces-like microorganisms. Also, earlier observational studies have noted an increased occurrence of BRONJ in patients with periodontal disease.[110,120,121] Recently, a few studies have noted BRONJ lesions in animals utilizing periodontal disease as a comorbid factor.[122,123] Furthermore, BRONJ lesions respond well to antibiotics and antimicrobial mouth rinses. $(102,[103]$ Collectively, these findings suggest that periodontal infection may play a role in BRONJ development. In order to test this hypothesis, we will undertake a case-control clinical study investigating the abovementioned specific aims. 


\section{Specific Aim 3:}

Specific aim 3a: To evaluate biomechanical properties of BP treated rat mandible and femur

Specific aim 2b: To evaluate differences in biomechanical properties of different areas of $B P$ treated rat mandible

Hypothesis 3 (project 3): Long-term BP therapy alters biomechanical properties of jaw bone increasing the risk of BRONJ development.

Rationale: Severe suppression of osteoclasts by long-term BP therapy may disrupt delicate balance between bone resorption and bone formation. Studies suggest that high-dose BP therapy adversely affects bone deposition and bone remodeling resulting in brittle bone. Under constant masticatory forces, brittle jaw bones are prone to developing microfractures and microdamage resulting in BRONJ development. 


\section{CHAPTER 2}

A Retrospective Study Evaluating Frequency and Risk factors of Osteonecrosis of the Jaw in 576 Cancer Patients Receiving Intravenous Bisphosphonates 


\section{ABSTRACT}

Objective: To evaluate the frequency, risk factors, and clinical presentation of bisphosphonate (BP)-related osteonecrosis of the jaw (BRONJ).

Study Design: We performed a retrospective analysis of 576 patients with cancer treated with intravenous pamidronate and/or zoledronate between January 2003 and December 2007 at the University of Minnesota Masonic Cancer Center and Park Nicollet Institute.

Results: Eighteen of 576 identified patients (3.1\%) developed BRONJ including 8 of 190 patients $(4.2 \%)$ with breast cancer, 6 of 83 patients $(7.2 \%)$ with multiple myeloma, 2 of 84 patients $(2.4 \%)$ with prostate cancer, 1 of 76 patients $(1.3 \%)$ with lung cancer, 1 of 52 patients $(1.9 \%)$ with renal cell carcinoma, and in none of the 73 patients with other malignancies. Ten patients (59\%) developed BRONJ after tooth extraction, whereas 7 (41\%) developed it spontaneously (missing data for 1 patient). The mean number of BP infusions $(38.1 \pm 19.06$ infusions vs. $10.5 \pm 12.81$ infusions; $\mathrm{P}<0.001)$ and duration of BP treatment $(44.3 \pm 24.34$ months vs. $14.6 \pm 18.09$ months; $\mathrm{P}<0.001$ ) were significantly higher in patients with BRONJ compared with patients without BRONJ. Multivariate Cox proportional hazards regression analysis showed that diabetes [hazard ratio $(\mathrm{HR})=3.40 ; 95 \%$ confidence interval $(\mathrm{CI}), 1.14$ $10.11 ; \mathrm{P}=0.028]$, hypothyroidism $(\mathrm{HR}=3.59 ; 95 \% \mathrm{CI}, 1.31-9.83 ; \mathrm{P}=0.013)$, smoking $(\mathrm{HR}=3.44 ; 95 \% \mathrm{CI}, 1.28-9.26 ; \mathrm{P}=0.015)$, and higher number of zoledronate infusions $(\mathrm{HR}=1.07 ; 95 \% \mathrm{CI}, 1.03-1.11 ; \mathrm{P}=0.001)$ significantly increased the risk of developing BRONJ. 
Conclusions: Increased cumulative doses and long-term BP treatment are the most important risk factors for BRONJ development. Type of BP, diabetes, hypothyroidism, smoking, and prior dental extractions may play a role in BRONJ development. 


\section{Introduction}

Bisphosphonates (BP) are pyrophosphate analogs with high affinity for hydroxyapatite crystals.[1,2,3] Once bound to hydroxyapatite crystals, BP are slowly released during bone resorption and subsequently internalized by osteoclasts.[27] BP inhibit osteoclastic bone resorption by interfering with osteoclast recruitment[28], differentiation[124], activity[27,30] and by promoting apoptosis.[31,32] In addition, $\mathrm{BP}$ are reported to have direct antiproliferative and proapoptotic effects on cancer cells thereby reducing bone metastases.[16,17,125,126] Furthermore, BP alter angiogenesis[33,34] and signal transduction between osteoclasts and osteoblasts.[35] Thus, BP play a key role in managing osteolytic bone disorders including osteoporosis, Paget's disease, bone metastasis and multiple myeloma.[9,12,22,23,127]

Bisphosphonate-related osteonecrosis of the jaw (BRONJ) is a well recognized complication associated with BP therapy.[84,86,97,102,103] BRONJ presents as exposed bone in the maxillofacial area showing delayed signs of healing. These lesions may be asymptomatic or present with pain, swelling, purulent discharge, tooth mobility and paresthesia.[84,86,97,102,103] The majority of reported BRONJ cases $(97 \%)$ are related to high-dose intravenous BP therapy in cancer patients.[20,84,86,97,102,103] At present, there is no curative therapy for BRONJ; therefore, patients are treated palliatively with antibiotics, antimicrobial mouth rinses and debridement of necrotic bone in an attempt to limit the extent of the condition. 
The true incidence, etiology and risk factors that contribute to BRONJ pathogenesis are unknown. The resulting knowledge gap has impaired the determination of individual susceptibility and development of preventative and therapeutic modalities for BRONJ. We conducted a multi-center retrospective study to determine the frequency, risk factors and clinical presentation of BRONJ in cancer patients treated with intravenous BP.

\section{Materials and Methods}

The Institutional Review Board at University of Minnesota (UMN) and Park Nicollet Institute (PNI) approved the study and waived the requirement for obtaining informed consent.

\section{Study Design}

Patients treated with intravenous BP therapy between January 2003 and December 2007 at the UMN Masonic Cancer Center and PNI were identified through respective institute's pharmacy databases. Out of the 814 medical charts identified, 238 records were excluded due to ineligibility and/or incomplete data. The study dates of 20032007 were selected as they permitted the incorporation of multidisciplinary records (pharmacy, medical and dental records) from both institutes.

Inclusion criteria: All patients who had received at least one dose of intravenous BP for cancer management were included in this study. Pamidronate was administered at $90 \mathrm{mg}$ as a 2-hour infusion every $4-5$ weeks and zoledronate was administered at 4 
mg every 4-6 weeks over 15 minutes. BP dose adjustment was done based on patient's calcium level and/or renal function.

Exclusion criteria: Patients with a history of either radiation therapy to the head and neck region or neoplasm (including metastasis) involving the head and neck region were excluded from the study. Patients who had received intravenous BP for osteoporosis management or Paget's disease were excluded.

\section{Definition and diagnosis of BRONJ}

The BRONJ definition provided by the American Association of Oral and Maxillofacial Surgeons (AAOMS) was used in this study.[85] BRONJ was defined as "exposed bone in the maxillofacial area occurring in the absence of head and neck irradiation and showing no evidence of healing for at least 8 weeks after identification in patients treated with BP therapy". Suspicious BRONJ lesions were identified through the medical records containing a documentation of exposed bone or osteomyelitis or delayed healing in the jaw bones.

\section{Data Collection}

The following data were collected from medical records: demographics, type of cancer and treatment received, co-morbid conditions with respective treatments, indication for BP treatment and details regarding BP therapy (type, dose, and duration). In addition, the following information was abstracted from dental records of BRONJ patients referred to the dental school for BRONJ management: date of 
BRONJ diagnosis, associated signs and symptoms, location, inciting events, treatments received and outcomes.

\section{Statistical analysis}

We performed a retrospective analysis of 576 patients treated with pamidronate and/or zoledronate. The duration of BP exposure was defined as time in months from the date of first BP infusion administered to the last recorded infusion. Time to develop BRONJ was determined as time in months from the date of first BP infusion administered to the date of BRONJ diagnosis. We performed univariate analyses (Fisher's exact tests for categorical variables and t-tests for continuous variables) to compare baseline characteristics between BRONJ and non-BRONJ patients. Stepwise logistic regression was used to investigate multivariate associations with the development of BRONJ. Stepwise Cox's proportional hazards regression was used to investigate multivariate associations with time to development of BRONJ. For

multivariate analysis, covariates with p-values $<0.20$ were entered into the model and covariates with $\mathrm{p}$-values $<0.10$ were retained in the reduced model. Characteristics outlined in Table 1 and Table 2 were considered for inclusion in the regression models. Kaplan-Meier method was used to estimate the median BRONJ-free time. Pvalues $<0.05$ were deemed statistically significant. SAS V9.1.3 (SAS Institute, Cary, NC) was used for the analysis. 


\section{Results}

\section{Patient Characteristics}

A total of 18 BRONJ cases (3.1\%) were identified. The baseline characteristics of 576 eligible patients including 18 BRONJ patients are listed in Table 1. There were no differences in BRONJ incidence rate between UMN and PNI population. BRONJ was noted in $8(4.2 \%)$ of the 190 patients with breast cancer, $6(7.2 \%)$ of the 83 patients with multiple myeloma, $2(2.4 \%)$ of the 84 patients with prostate cancer, 1 (1.3\%) of the 76 patients with lung cancer, $1(1.9 \%)$ of the 52 patients with renal cell carcinoma and in none of the 73 patients with other malignancies. Forty four percent of BRONJ patients had a history of smoking ( $>10$ years) and $22 \%$ had a history of chronic alcohol consumption. In addition to cancer, patients had at least one of the medical conditions listed in Table 1 and had been respectively treated with antihypertensive medications, anticoagulants, insulin, iron and thyroid supplements. Furthermore, eighty three percent of BRONJ patients had received pulsed corticosteroids and $33 \%$ had received antiangiogenic agents.

\section{Bisphosphonate Exposure}

BRONJ development was significantly associated with type of BP, number of BP infusions and duration of BP therapy ( $\mathrm{P}<0.05$, Table 2). Of the 576 patients, 412 patients $(71 \%)$ were treated with zoledronate, 88 patients $(15 \%)$ with pamidronate and 76 patients $(13 \%)$ with pamidronate plus zoledronate sequentially. BRONJ patients had received significantly higher mean number of BP infusions $(38.1 \pm 19.06$ infusions versus $10.5 \pm 12.81$ infusions, $\mathrm{p}<0.001$ ) and longer duration of BP therapy 
$(44.3 \pm 24.34$ months versus $14.6 \pm 18.09$ months, $\mathrm{p}<0.001)$ compared to patients without BRONJ. In addition, combination of pamidronate plus zoledronate treatment was more frequently noted in BRONJ patients compared to patients without BRONJ $(39 \%$ versus $13 \%, p=0.008)$.

\section{BRONJ Characteristics and BRONJ Management}

BRONJ was noted more frequently in the mandible $(76 \%)$ than the maxilla $(23 \%)$ (Table 3). The posterior mandible was the most frequently affected site. While some patients $(59 \%)$ developed BRONJ following dentoalveolar treatment, others $(41 \%)$ developed it without an obvious precipitating event. In most patients, pain was the common presenting symptom, while some exhibited evidence of erythema, infection with or without purulence.

BRONJ management and treatment outcomes are reported elsewhere.[128] Briefly, BRONJ management was directed towards alleviating pain, reducing lesion size, soft and hard tissue inflammation and/or infection. In most patients, initial treatment was non-surgical and included antibiotics, antifungal agents, and rinsing or irrigating the lesion with an antimicrobial solution (chlorhexidine gluconate). Debridement without mucosal elevation was performed at subsequent visits if needed. Irrespective of the disease stage, loose segments of bony sequestra were removed. Patients were instructed to improve their oral hygiene and measures were taken to adjust any illfitting dentures if present. Following BRONJ treatment, lesions healed completely in 2 patients $(11 \%)$, healed partially in $5(28 \%)$, remained stable in $5(28 \%)$ and progressed in $6(33 \%)$. 


\section{Risk factors associated with BRONJ Development}

We examined several factors including age, gender, type of malignancy, pre-existing medical conditions, environmental factors, prior medications and type of BP, number of BP infusions and duration of BP therapy. There were no significant differences in age $(\mathrm{P}=0.337)$ or gender $(\mathrm{P}=0.634)$ or type of malignancy $(\mathrm{P}=0.187)$ between patients with or without BRONJ (Table 1).

Univariate analysis showed that hypothyroidism $(\mathrm{p}=0.002)$, alcohol $(\mathrm{p}=0.019)$, steroid use $(p=0.049)$, thyroxine-replacement $(p=0.026)$, type of $B P(p=0.008)$, number of $B P$ infusions $(p<0.001)$ and duration of BP therapy $(p<0.001)$ were associated with BRONJ development (Table 1 and Table 2). These factors were further analyzed using a stepwise logistic regression analysis to investigate multivariate associations with BRONJ development. Regression analysis revealed diabetes $(\mathrm{OR}=4.12 ; 95 \%$ $\mathrm{CI}=1.08-15.75 ; \mathrm{p}=0.039)$, hypothyroidism $(\mathrm{OR}=5.30 ; 95 \% \mathrm{CI}=1.47-19.08 ; \mathrm{p}=0.011)$, smoking $(\mathrm{OR}=4.97 ; 95 \% \mathrm{CI}=1.43-17.23 ; \mathrm{p}=0.012)$ and number of zoledronate infusions $(\mathrm{OR}=1.15 ; 95 \% \mathrm{CI}=1.10-1.20 ; \mathrm{p}<0.001)$ to be significantly associated with BRONJ development (Table 4). Notably, diabetes appeared significant in the multivariate analysis after adjusting for other variables in the model. Since the number of zoledronate infusions is associated with the occurrence of BRONJ, having non-diabetics with a higher number of zoledronate infusions seems to be masking the association between diabetes and BRONJ in the univariate analysis.

To further investigate associations between these variables and time to develop BRONJ, stepwise Cox's proportional hazards regression was performed. Diabetes 
$(\mathrm{HR}=3.40 ; 95 \% \mathrm{CI}=1.14-10.11 ; \mathrm{p}=0.028)$, hypothyroidism $(\mathrm{HR}=3.59 ; 95 \% \mathrm{CI}=1.31-$ 9.83; $\mathrm{p}=0.013)$, smoking $(\mathrm{HR}=3.44 ; 95 \% \mathrm{CI}=1.28-9.26 ; \mathrm{p}=0.015)$ and number of zoledronate infusions $(\mathrm{HR}=1.07 ; 95 \% \mathrm{CI}=1.03-1.11 ; \mathrm{p}=0.001)$ showed significant association with time to development of BRONJ (Table 5).

Relationship between type of BP and median BRONJ-free time was further explored using Kaplan Meier survival analysis. There were no significant differences in the probability of being BRONJ free between pamidronate, zoledronate and pamidronate plus zoledronate groups (Figure 1). The small number of BRONJ patients limited the determination of long term hazard of developing BRONJ. The time to development of BRONJ ranged from 7.2 months to 104.6 months. BP was discontinued in 11 patients prior to or at BRONJ diagnosis, whereas the remaining 7 patients continued BP for a median of 4.8 months.

\section{Discussion}

While numerous reports have presented BRONJ cases, only a few have investigated the incidence and risk factors for BRONJ development.[86,97,129] In addition, the wide disparity in incidence and risk factors among these studies are attributable to variations in BP treatment regimen (type, dose, duration), nature of study (single center studies evaluating subset of cancer population), age, status of disease and cancer management. The present study differs from most of the previously published reports as it is a multi-center study evaluating the BRONJ 
frequency and risk factors in a large cohort of population that is not restricted to multiple myeloma or breast cancer groups.

We noted an overall BRONJ frequency of 3.1\% (7.2\% in multiple myeloma, $4.2 \%$ in breast cancer and $2.4 \%$ in prostate cancer). These percentages are similar to those reported in other studies where an overall BRONJ frequency of $\sim 5 \%$ (range $0.72 \%$ to $7.4 \%$ ) was noted in cancer patients treated with intravenous BP.[86,94,95,97] Hoff et al. in their analysis of 4019 patients reported an overall BRONJ frequency of $0.72 \% .[97]$ The authors reported a higher BRONJ frequency in multiple myeloma patients $(2.4 \%)$ compared to breast cancer patients $(1.2 \%)$. Similarly, other studies have also reported a higher BRONJ frequency in multiple myeloma patients (ranging from $1.7 \%$ to $11 \%$ ) compared to breast cancer patients (ranging from $0.6 \%$ to $6.2 \%$ ). $[86,87,95,97,111,129,130,131,132]$ The differences among numerous reported studies, including ours, are most probably due to variations in sample size, varying methods of data assessment/analysis and limitations due to voluntary case reporting. Also, previous studies indicate that BRONJ frequency may vary according to geographical locations reflecting the potential influence of genetic background and differences in oral health in the development of BRONJ.[86,94,95,97,111,129,130,131,132]

Previous studies have reported that the type of BP may play a role in BRONJ development, particularly the nitrogen-containing BP like pamidronate and 
zoledronate.[20,84,85,86,87,91,97,102,103,129] Bamias et al. and others have suggested that the risk is higher with zoledronate than pamidronate.[86,87,97,129] In agreement with these reports, we noted that the hazard of developing BRONJ was significantly higher with zoledronate infusions compared to pamidronate or pamidronate plus zoledronate infusions. The reason for this difference between the two types of BP is unknown. A plausible explanation is the more potent inhibitory effect on bone turnover and stronger anti-resorptive activity of zoledronate compared to pamidronate. Zoledronate is $10-100$ times more potent than pamidronate.[2] The significant impairment in bone turnover rate caused by continuous administration of zoledronate may eventually lead to increased bone fragility and in combination with other factors increase the risk of BRONJ development.

Our analysis demonstrated that BRONJ development was significantly associated with longer duration (months, $44.3 \pm 24.34$ vs $14.6 \pm 18.09, \mathrm{p}<0.001$ ) and increased number of BP infusions $(38.1 \pm 19.06$ vs $10.5 \pm 12.81, \mathrm{p}<0.001)$ (Table 2). We further confirmed by multivariate analysis that number of zoledronate infusions is an independent risk factor for BRONJ development. These results are consistent with previous studies which have noted that prolonged duration of BP exposure is a significant risk factor in the development of BRONJ.[86,87,97,102,129] In addition, BRONJ was more frequently noted in multiple myeloma and breast cancer patients compared to prostate cancer, lung cancer, renal cell carcinoma and other neoplasm group, although not statistically significant. The disparity in BRONJ incidence 
between multiple myeloma, breast cancer and other groups is most likely due to the differences in $\mathrm{BP}$ infusions and $\mathrm{BP}$ exposure time between these groups.

Stepwise Cox's proportional hazards regression analysis showed that diabetes, hypothyroidism, smoking, and number of zoledronate infusions was significantly associated with time to development of BRONJ (Table 5). Most of these factors except hypothyroidism have previously reported to be associated with osteonecrosis development. [86,119,133]

Thyroid hormone is essential for skeletal development and establishment of peak bone mass. In adults, $\mathrm{T} 3$ regulates bone turnover and bone mineral density and normal euthyroid status is essential to maintain optimal bone strength. Several studies indicate that hypothyroidism is associated with increased risk of fracture.[134,135,136] In addition, a histometric study in rats noted that hypothyroidism resulted in reduced new bone formation between dental implant threads and decreased bone quality adjacent to the implant surface.[137] Furthermore, an experimental animal study noted that the state of hypothyroidism decreased typeIV collagen and hydroxyproline level during the proliferative phase of wound healing.[138] Collectively, these factors suggest that state of hypothyroidism constitutes an important factor in delayed wound healing.

The present study primarily focuses on evaluating the frequency, risk factors and clinical presentation of BRONJ. The management of BRONJ patients and treatment outcomes are reported elsewhere.[128] Consistent with previous reports we found the mandible and posterior aspect of the jaws to be the most common site for 
BRONJ development. Our study also confirmed other published reports that tooth extraction is the most common inciting factor for BRONJ development. However, the risk of developing BRONJ as a consequence of dental extraction could not be further assessed due to the limited access to dental records of Non-BRONJ patients.

The retrospective nature of the present study might have had an inherent bias and certain limitations. However, the results are consistent with those of the previously published studies. The overall frequency of $3.1 \%$ noted in this study is likely to be an underestimated value, since this was a retrospective study which relied on pre-existing medical records that did not specifically focus on oral tissues. While it is very likely that severe cases of BRONJ would be noticed by the physicians, a number of BRONJ cases might have gone undiagnosed unless the patients were specifically questioned for BRONJ symptoms using sensitive methods or patients underwent intra-oral examination. Nevertheless, this number is likely to be small, given that the oncologists at both the institutes routinely advised patients on BP therapy to undergo frequent dental examinations. Notably, most of the BRONJ patients identified through the medical records had been referred to the UMN dental school for BRONJ management.

In conclusion, results from the present study indicate that BRONJ development is associated with long-term BP use. The frequency of BRONJ appears to be higher in multiple myeloma and breast cancer groups compared to other malignancies, which is likely due to differences in BP treatment between these 
groups. Number of BP infusions, diabetes and history of chronic smoking are significant risk factors for BRONJ development. Lastly, we also report a novel finding that preexisting hypothyroidism might contribute towards BRONJ development. While this finding needs to be confirmed in future studies, there are plausible reasons why this association may be causal and not coincidental in nature. Future studies should clarify the role of thyroid status and the risk of other comorbidities in BRONJ development. 
Table 1. Patient Characteristics $(n=576)$

\begin{tabular}{|c|c|c|c|}
\hline Characteristic & $\begin{array}{l}\text { BRONJ } \\
(\mathrm{N}=18)\end{array}$ & $\begin{array}{l}\text { Non-BRONJ } \\
(\mathrm{N}=558)\end{array}$ & $P \dagger$ \\
\hline Age - mean (sd), in years & $60(11.5)$ & $63(13.5)$ & 0.337 \\
\hline $\begin{array}{l}\text { Sex }- \text { no. }(\%) \\
\text { Male } \\
\text { Female }\end{array}$ & $\begin{array}{l}6(33) \\
12(67)\end{array}$ & $\begin{array}{l}222(40) \\
336(60)\end{array}$ & 0.634 \\
\hline $\begin{array}{l}\text { Location - no. }(\%) \\
\text { University of Minnesota } \\
\text { Park Nicollet Institute }\end{array}$ & $\begin{array}{l}6(33) \\
12(67)\end{array}$ & $\begin{array}{l}178(32) \\
380(68)\end{array}$ & 1.000 \\
\hline $\begin{array}{l}\text { Malignant Disease - no. (\%) } \\
\text { Breast cancer } \\
\text { Multiple myeloma } \\
\text { Prostate cancer } \\
\text { Lung cancer } \\
\text { Renal cell carcinoma } \\
\text { Others }\end{array}$ & $\begin{array}{l}8(44) \\
6(33) \\
2(11) \\
1(6) \\
1(6) \\
0(0)\end{array}$ & $\begin{array}{l}190(34) \\
83(15) \\
84(15) \\
76(14) \\
52(9) \\
73(13)\end{array}$ & 0.187 \\
\hline $\begin{array}{l}\text { Pre-existing medical conditions - no. (\%) } \\
\text { Anemia } \\
\text { Diabetes mellitus } \\
\text { Deep-vein thrombosis/Pulmonary embolism } \\
\text { Hypertension } \\
\text { Hypercholesterolemia/Hyperlipidemia } \\
\text { Hypothyroidism } \\
\text { Renal impairment } \\
\text { Stroke } \\
\text { Thrombocytopenia }\end{array}$ & $\begin{array}{l}1(6) \\
5(28) \\
3(17) \\
11(61) \\
7(39) \\
7(39) \\
2(11) \\
1(6) \\
1(6)\end{array}$ & $\begin{array}{l}89(16) \\
87(16) \\
68(12) \\
240(43) \\
120(22) \\
58(10) \\
53(10) \\
14(3) \\
31(6)\end{array}$ & $\begin{array}{l}0.333 \\
0.186 \\
0.477 \\
0.150 \\
0.088 \\
0.002 \\
0.687 \\
0.383 \\
1.000\end{array}$ \\
\hline $\begin{array}{l}\text { Environmental factors }- \text { no. }(\%) \\
\text { Smoking } \\
\text { Alcoholism }^{\#}\end{array}$ & $\begin{array}{l}8(44) \\
4(22)\end{array}$ & $\begin{array}{l}133(24) \\
31(6)\end{array}$ & $\begin{array}{l}0.054 \\
0.019\end{array}$ \\
\hline $\begin{array}{l}\text { Previous medications } \\
\text { Antihypertensive } \\
\text { Anticoagulants } \\
\text { Corticosteroids } \\
\text { Antiangiogenic agents* } \\
\text { Thyroxine-replacement } \\
\text { Chemotherapy } \\
\text { Alkylating agents } \\
\text { Anti-metabolites } \\
\text { Vinca Alkaloids } \\
\text { Taxanes } \\
\text { Antitumor antibiotics } \\
\text { Hormonal therapy } \\
\text { Immunotherapy }\end{array}$ & $\begin{array}{l}11(61) \\
6(33) \\
15(83) \\
6(33) \\
4(22) \\
17(94) \\
5(28) \\
4(22) \\
3(17) \\
9(50) \\
5(28) \\
8(44) \\
2(11)\end{array}$ & $\begin{array}{l}151(47) \\
83(26) \\
326(58) \\
102(30) \\
19(6) \\
546(98) \\
133(41) \\
93(29) \\
55(17) \\
114(36) \\
47(15) \\
100(31) \\
92(16)\end{array}$ & $\begin{array}{l}0.333 \\
0.582 \\
0.049 \\
0.794 \\
0.026 \\
0.341 \\
0.327 \\
0.789 \\
1.000 \\
0.219 \\
0.170 \\
0.298 \\
0.751\end{array}$ \\
\hline
\end{tabular}




\section{Table 1 Footnote:}

† P-values were calculated from a Fisher's-exact test or a t-test for categorical or continuous variables, respectively.

" Treatment consisted of Diuretics, Adrenergic receptor antagonist, $\beta$ blockers, Calcium channel blockers, and ACE inhibitors.

$\S$ Pulsed corticosteroid treatment included dexamethasone and/or prednisone.

* Antiangiogenic treatment included bevacizumab and/or thalidomide.

${ }^{\int}$ Data analyzed as current/former smoker versus non-smoker.

\# Data analyzed as alcohol intake versus no alcohol intake. 
Table 2. Bisphosphonate Treatment $(n=576)$

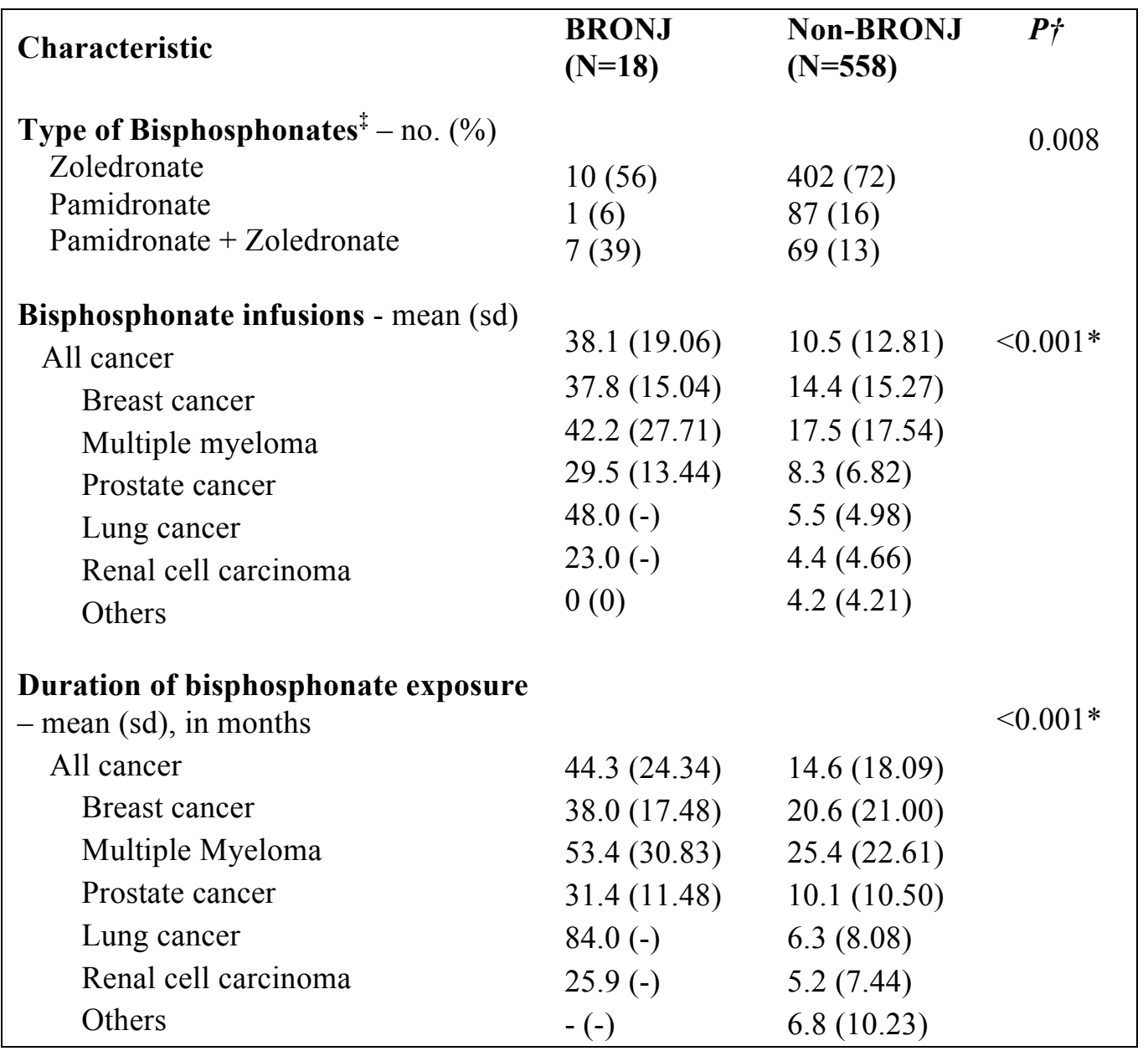

${ }^{*} P$ values were calculated from a Fisher exact test or a $t$ test for categorical or continuous variables, respectively.

${ }^{\dagger}$ Pamidronate + zoledronate versus pamidronate $(\mathrm{OR}=8.83 ; 95 \% \mathrm{CI}=1.06,73.5) ;$ zoledronate versus pamidronate $(\mathrm{OR}=2.16 ; 95 \% \mathrm{CI}=0.27,17.13)$.

${ }^{\ddagger}$ Log-scale.

BRONJ indicates bisphosphonate-related osteonecrosis of the jaw; CI, confidence interval; OR, odds ratio. 


\section{Table 3. BRONJ Characteristics}

\begin{tabular}{ll}
\hline $\begin{array}{l}\text { Characteristics } \\
\text { Location }^{\dagger}-\text { no. }(\%)\end{array}$ & BRONJ (N=18) \\
Maxilla & $4(23)$ \\
Mandible & $13(76)$ \\
Anterior sextant & $3(18)$ \\
Posterior sextant & $14(82)$ \\
BRONJ onset & $\dagger$ no. (\%) \\
Tooth extraction & $10(59)$ \\
Spontaneous & $7(41)$
\end{tabular}

${ }^{\dagger}$ Missing data for one subject 
Table 4. Risk Factors ${ }^{\dagger}$ for Developing BRONJ

\begin{tabular}{|llll|}
\hline Parameter & Odds Ratio & $\mathbf{9 5 \%}$ CI & $\boldsymbol{P}$ \\
Diabetes & 4.12 & $1.08-15.75$ & 0.039 \\
Hypothyroidism & 5.30 & $1.47-19.08$ & 0.011 \\
Smoking & 4.97 & $1.43-17.23$ & 0.012 \\
Steroids & 3.46 & $0.79-15.16$ & 0.099 \\
Zoledronate infusions & & $1.10-1.20$ & $<0.001$ \\
Pamidronate infusions $^{\S}$ & 1.15 & $0.995-1.07$ & 0.093 \\
\hline
\end{tabular}

${ }^{\dagger}$ Identified using a stepwise logistic regression procedure.

" Odds ratio represents current/former smoker versus non-smoker.

$\S$ Odds ratio represents increase in risk per infusion. 
Table 5. Risk Factors ${ }^{\dagger}$ for Time to Development of BRONJ

\begin{tabular}{|llll|}
\hline Parameter & Hazard Ratio & $\mathbf{9 5 \%}$ CI & $\boldsymbol{P}$ \\
Diabetes & 3.40 & $1.14-10.11$ & 0.028 \\
Hypothyroidism & 3.59 & $1.31-9.83$ & 0.013 \\
Smoking & 3.44 & $1.28-9.26$ & 0.015 \\
Zoledronate infusions $^{\S}$ & 1.07 & $1.03-1.11$ & 0.001 \\
\hline
\end{tabular}

${ }^{\dagger}$ Identified using a stepwise proportional hazards regression procedure.

${ }^{\natural}$ Hazard ratio represents current/former smoker versus non-smoker.

${ }^{\S}$ Hazard ratio represents increase in risk per infusion. 


\section{Figure 1.}

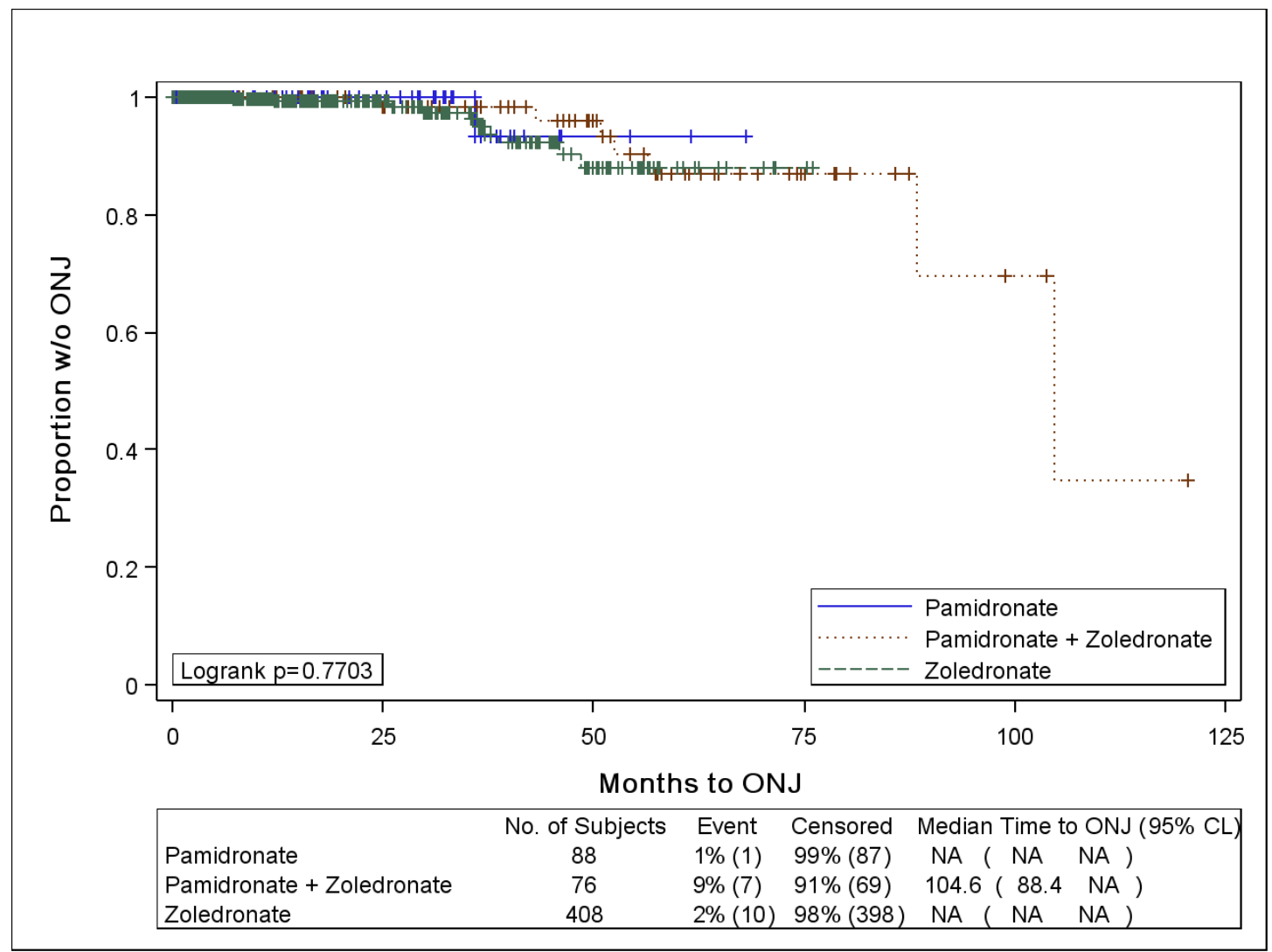

Kaplan-Meier estimates of the proportion of patients without bisphosphonate-related osteonecrosis of the jaw over time according to type of bisphosphonate therapy received. 


\section{CHAPTER 3}

Periodontal Disease and Bisphosphonate-Related Osteonecrosis of the Jaw 


\section{Abstract}

Background: Previous reports suggest that poor oral hygiene predisposes patients to developing Bisphosphonate-Related Osteonecrosis of the Jaw (BRONJ). We conducted a case-control study to evaluate the association between clinical and radiographic measures of periodontal disease and BRONJ.

Methods: 25 BRONJ patients were matched with 48 controls. For each subject, one of two calibrated examiners measured probing depth (PD), clinical attachment loss (CAL), and bleeding on probing (BOP) at six sites on all teeth except third molars, and measured gingival index (GI) and plaque index (PI) on six index teeth. Alveolar bone height was measured for each subject using panoramic radiograph. As part of BRONJ management, most cases were using antibiotics $(48 \%)$ or chlorhexidine mouth rinse $(84 \%)$ at the time of enrolment.

Results: BRONJ cases had significantly more missing teeth than controls (mean, 9.7 vs 3.1; $\mathrm{p}=<0.001)$. In the unadjusted analysis, BRONJ cases and controls did not differ significantly in average PD (2.07 vs $2.11 \mathrm{~mm}, \mathrm{p}=0.60)$, percent of sites with BOP ( 12.0 vs $14.9, \mathrm{p}=0.42)$, average GI $(0.54$ vs $0.58, \mathrm{p}=0.62)$, average PI ( 0.40 vs $0.55, \mathrm{p}=0.13)$, or percent of sites with probing depth $4-6 \mathrm{~mm}(5.0$ vs $4.8, \mathrm{p}=0.87)$. However, they differed significantly for average CAL (2.18 vs $1.56 \mathrm{~mm}, \mathrm{p}=0.047)$, percent of sites with PD $>6 \mathrm{~mm}(0.49$ vs $0.03, \mathrm{p}=0.018)$, and percent of sites with $\mathrm{CAL} \geq 3 \mathrm{~mm}$ (39.0 vs $23.3, \mathrm{p}=0.039)$. After adjusting for age, sex and smoking status, cases and controls differed significantly for percent of sites with $\mathrm{CAL} \geq 3 \mathrm{~mm}$ (38.0 vs $26.9, \mathrm{p}=0.056)$ including tooth loss $(9.2$ vs $3.8 ; \mathrm{p}=<0.001)$. Radiographs reveled BRONJ subjects had significantly lower residual alveolar bone support compared to 
controls (mean full-mouth bone score, 0.59 vs $0.62, \mathrm{p}=0.004$ ). Around $20 \%$ of the BRONJ cases had more than $50 \%$ alveolar bone loss compared to $6 \%$ of the controls $(\mathrm{P}=0.001)$.

Conclusions: Poor periodontal status is noted among BRONJ subjects. Imbalances in antibiotics and chlorhexidine mouth rinse usage between the groups may have eliminated or reduced differences in GI, PI, and PD in the present study. The role of poor oral hygiene in BRONJ development needs to be validated in future prospective studies. 


\section{Introduction}

Previous reports suggest that poor oral hygiene predisposes patients to developing Bisphosphonate-Related Osteonecrosis of the Jaw (BRONJ). In one of the earliest studies recognizing BRONJ condition, Marx et al. noted that $84 \%$ of their BRONJ patients had periodontal disease including 29\% with advanced periodontal disease.[102] Ficarra et al. reported a series of nine patients in whom BRONJ was noted following extraction of periodontally "hopeless" teeth.[110] Furthermore, periodontal pathogens have been isolated from BRONJ lesions. Badros et al. identified putative periodontal pathogens (Prevotella and Porphyromonas species) in 9 out of 17 BRONJ patients.[106] Lesions in the remaining 8 patients contained Actinomyces species. Likewise, Sedghizadeh et al. showed that bone sections from BRONJ sites contained microbial biofilms comprised predominantly of bacteria such as Fusobacterium, Actinomyces, Staphylococcus, Streptococcus, Selenomonas and Trepenemes.[114] The contribution of these organisms to BRONJ development is unknown, and it is vital to determine whether bacterial colonization contributes to BRONJ development, or represents secondary infection.

Recently, a few animal studies have attempted to develop BRONJ model utilizing periodontal disease as a comorbid factor.[122,123] Aghaloo et al. noted that, following induction of aggressive periodontal disease by ligature placement around the crown of maxillary first molar, $47 \%$ of the zoledronate treated rats showed histological and radiological evidence of osteonecrosis, while $21 \%$ showed evidence of exposed bone.[122] Similarly, Aguirre et al. noted that high dose zoledronate 
administration exacerbated inflammatory response and periodontal tissue damage in rice rats, inducing BRONJ-like lesions.[123] Rice rats treated with high doses of zoledronate for 18 to 24 weeks developed gross lesions in the mandible (40\%) and maxilla (20\%). These lesions were characterized by areas of exposed necrotic bone, presence of bacterial colonies, and periodontal tissue destruction. In addition, inhibition of bone formation, increased osteocyte apoptosis, and decreased blood vessel number were noted.

An association between periodontal disease and BRONJ development has been suggested in various publications.[20,84,106,111] To date, no epidemiologic studies have evaluated the role of periodontal disease in BRONJ. We conducted a casecontrol study to evaluate the association between clinical and radiographic measures of periodontal disease and BRONJ development.

\section{Patients and Methods}

\section{Subjects}

The University of Minnesota Institutional Review Board approved this case-control study and written informed consent was obtained from all subjects. Cancer patients above 30 years of age who had received intravenous BP therapy for cancer management were included in this study. Patients with a history of either radiation therapy to the head and neck region or neoplasm's (including metastasis) involving the head and neck region were excluded. Eligible case patients included those with a 
confirmed diagnosis of BRONJ. The American Association of Oral and Maxillofacial Surgeons criteria [85] was used to diagnose BRONJ. Briefly, BRONJ was defined as "exposed bone in the maxillofacial area occurring in the absence of head and neck irradiation and showing no evidence of healing for at least 8 weeks after identification in patients treated with BP therapy". The control group consisted of cancer patients who had received at least 10 intravenous BP infusions without a history of BRONJ.

At enrollment, all study subjects underwent a comprehensive dental examination and periodontal examination including oral cancer screening. For each subject, one of two calibrated examiners measured probing depth (PD), clinical attachment loss (CAL), and bleeding on probing (BOP) at six sites on all teeth except third molars, and measured gingival index (GI) and plaque index (PI) on six index teeth. Alveolar bone height was measured using panoramic radiographs. Digital radiographs or radiographic films converted into digital format were analyzed using Adobe Photoshop CS3 version 10 (Adobe, CA, USA). Mesial and distal bone heights (measured in pixels) were determined as a proportion of tooth length as described previously.[139] A full-mouth bone score was computed for each subject by averaging mesial and distal proportions from all measurable teeth.

\section{Statistical Analysis}

Baseline comparisons used Fisher's exact test or Pearson's chi-squared test for categorical variables (e.g., type of malignant disease) and two-sample t-tests for 
quantitative measurements. Unadjusted comparisons of outcome measures used twosample t-tests; adjusted comparisons used multiple linear regression analysis, adjusting for sex, age, and smoking (ever vs never). Three subjects with BRONJ were edentulous and thus had no data for clinical periodontal measures or bone loss. To include these three subjects in the analyses, they were assigned the worst possible score for each outcome and then, for each outcome, the adjusted analyses were redone using as the dependent variable each person's rank according to the outcome measure. This approach is a generalization of popular non-parametric tests such as the Wilcoxon rank sum test.[140] For all outcomes, the analyses excluding and including the edentulous subjects gave qualitatively identical results, so we present only the P-values from the analysis including the edentulous subjects.

\section{Results}

\section{Patient Characteristics}

A total of 25 BRONJ cases and 48 controls participated this study. Table 1 lists select subject characteristics. There were no significant differences in age $(\mathrm{P}=0.50)$, sex $(\mathrm{P}=0.60)$, race (1.00), or smoking status $(\mathrm{P}=0.11)$ between $\mathrm{BRONJ}$ cases and controls. In addition, there was no significant difference in diabetes mellitus $(\mathrm{P}=0.66)$ or corticosteroid use $(\mathrm{P}=0.45)$ between the two groups. All study subjects had received zoledronate, pamidronate, or a combination of pamidronate and zoledronate therapy. The mean number of BP infusions was significantly higher in BRONJ cases 
compared to controls (mean $\pm \mathrm{SD}, 38.4 \pm 26.3$ infusions versus $18.8 \pm 7.2 ; \mathrm{P}=0.0001$ ). BRONJ cases also received BP therapy for a longer period (45.1 \pm 29.7 months versus 37.7 \pm 21.9 months), although the difference was not significant $(\mathrm{P}=0.23)$. Most patients had received BP therapy for metastatic breast cancer management (13 BRONJ and 27 controls, $\mathrm{p}=0.02$ ).

Notably, at the time of sampling, BRONJ cases had discontinued BP therapy for a mean period of 11.4 months (2 to 30 months) following BRONJ diagnosis, while controls continued to receive BP therapy for cancer management.

\section{BRONJ Characteristics}

BRONJ was noted more frequently in the mandible (80\%) than the maxilla (28\%) with some subjects exhibiting lesions in both the jaws (Table 4). The posterior region of the mandible was the most frequently affected site (56\%). Tooth extraction was often the precipitating factor for BRONJ development (64\%), while some patients developed BRONJ spontaneously (24\%) without an obvious precipitating factor. Few patients also developed BRONJ following implant placement, root canal therapy, and crown preparation. As part of BRONJ management, most cases were using antibiotics $(48 \%)$ or chlorhexidine mouth rinse $(84 \%)$ at the time of enrolment. Antibiotics treatment mainly included penicillin, clindamycin, amoxicillin/clavulanate, and cephalexin. 


\section{Clinical Periodontal Measures}

Table 2 presents unadjusted comparisons of clinical periodontal measurements for BRONJ cases and controls. BRONJ cases had significantly more missing teeth than controls (mean, 9.7 vs $3.1 ; p=<0.001$ ). Three of the BRONJ subjects were completely edentulous. In the unadjusted analysis, BRONJ cases and controls did not differ significantly in average PD (2.07 vs $2.11 \mathrm{~mm}, \mathrm{p}=0.60)$, percent of sites with BOP (12.0 vs $14.9, \mathrm{p}=0.42)$, average GI $(0.54$ vs $0.58, \mathrm{p}=0.62)$, average PI ( 0.40 vs 0.55 , $\mathrm{p}=0.13$ ), or percent of sites with probing depth 4-6 mm (5.0 vs 4.8, $\mathrm{p}=0.87)$. However, they differed significantly for average CAL (2.18 vs $1.56 \mathrm{~mm}, \mathrm{p}=0.047)$, percent of sites with probing depth $>6 \mathrm{~mm}(0.49$ vs $0.03, \mathrm{p}=0.018)$, and percent of sites with $\mathrm{CAL} \geq 3 \mathrm{~mm}$ (39.0 vs $23.3, \mathrm{p}=0.039)$. After adjusting for age, sex and smoking status, cases and controls differed significantly for percent of sites with CAL $\geq 3 \mathrm{~mm}(38.0$ vs $26.9, \mathrm{p}=0.056)$ including tooth loss $(9.2$ vs $3.8 ; \mathrm{p}=<0.001)($ Table 3$)$.

\section{Radiographic Alveolar Bone Height}

Radiographs reveled BRONJ subjects had significantly lower residual alveolar bone support compared to controls (Table 5 and Table 6). The mean full-mouth bone score was significantly lower in BRONJ group compared to controls in both the unadjusted $(0.59$ vs $0.62, p=0.004)$ and adjusted analysis $(0.60$ vs $0.62, p=0.003)$. Around $20 \%$ of the BRONJ cases had more than $50 \%$ bone loss compared to $6 \%$ of the controls $(\mathrm{P}=0.001)$. 


\section{Discussion}

The present study noted increased clinical attachment loss and decreased alveolar bone support in BRONJ subjects who had received intravenous BP therapy. These findings are in agreement with the previous hypothesis that poor oral hygiene predisposes patients to developing BRONJ. Earlier observational studies have noted an increased incidence of BRONJ in patients with periodontal disease.[110,120,121] The risk of developing BRONJ is estimated to be around 7 -fold higher in patients with a history of periodontal infection and dental abscesses.[97] Furthermore, BRONJ lesions respond well to antibiotics and antimicrobial mouth rinses, which indicates the potential role of dental infection in BRONJ development.[103,120]

Numerous histological analyses illustrate that infection is almost a universal finding in BRONJ specimens.[114,141] Recently, an animal model evaluated the effects of Fusobacterium nucleatum, a prevalent periodontal pathogen, in BRONJ development.[142] In pamidronate treated rats, tooth extraction followed by oral infection with Fusobacterium nucleatum caused BRONJ-like lesions and delayed epithelial healing, both of which were completely suppressed by a broad-spectrum antibiotic cocktail. The combination of Fusobacterium nucleatum and pamidronate affected gingival fibroblast and keratinocyte growth factor (essential for epithelial regeneration), which resulted in delayed epithelial healing combined with bone death.

Bacteria are known to cause pathological bone remodeling.[143] The soluble virulence factors released by bacteria may affect skeletal structure by (i) destroying 
the noncellular components of bone by liberating acid and proteases; (ii) promoting cellular processes that stimulate the degradation of bone; and (iii) by inhibiting the synthesis of bone matrix. Several mechanisms have been suggested regarding the role of infection in BRONJ development.[122,123,144] Since osteoclast activity is diminished following BP therapy, in inflammatory conditions like periodontal disease, the alveolar bone may not be resorbed away from the inflammatory nidus. Consequently, the alveolar bone is exposed to an environment rich in bacterial toxins, inflammatory cytokines, or oxidative stresses that is highly noxious to bone and could potentially result in bone necrosis.[122] In contrast, it is suggested that infection induced aggressive local osteolysis may play a role in BRONJ development.[144] Bacteria have the capacity to directly resorb bone, independent of osteoclasts, by liberating various acids and proteases.[143] Increased bone turnover induced by bacterial inflammation may enhance the incorporation of BP's into inflammatory areas. Indeed, studies have noted increased BP uptake in the presence of periapical or periodontal infection.[145,146] Subsequently, the higher concentration of BP may exert direct cytotoxic effects on bone cells resulting in osteonecrosis. Furthermore, resorption that occurs independent of osteoclasts would likely lack osteoblastmediated bone formation as osteoclast signaling is essential for osteoblast function during bone remodeling.[147]

In addition, it is proposed that BRONJ may result from increased bacterial adhesion to bone surfaces coated with BPs.[141,148] An in vitro study noted that clodronate and pamidronate promoted bacterial colonization (Staphylococcus aureus) to the 
hydroxyapatite surface.[149] The protein named "MSCRAMM" (microbial surface components which recognize adhesive matrix molecules) mediates bacterial adhesion to the hydroxyapatite surface. In agreement with this theory, numerous studies have noted BRONJ sites to be heavily colonized with specific oral bacteria including Streptococcus, Eubacterium, Pseudoramibacter, Actinomyces, and Staphylococcus.[114,141] Finally, BP's immunomodulatory effect is suggested to have a role in BRONJ. It has been shown that after osteoclasts, monocytes and macrophages are the cells that are most likely affected by BP's.[150] Impaired monocyte and macrophage function may increase the risk of local infection like osteomyelitis. As BP's do not affect neutrophils, the risk of systemic infection is minimal.

Similar to our study, a retrospective study by Carmagnola et al. noted reduced alveolar bone support in BRONJ subjects compared to non-BRONJ subjects, although the difference was statistically insignificant.[151] A small sample size may have contributed to the insignificant differences in Carmagnola's study. In the present study we observed no significant difference in PI, GI, BOP and PD between BRONJ cases and controls. Imbalances in antibiotics and chlorhexidine mouth rinse usage between the groups may have eliminated or reduced the differences in clinical periodontal measurements. Lopez et al. noted that metronidazole plus amoxicillin therapy alone (without non-surgical or surgical therapy) reduced BOP by $26.5 \%$, mean PD by $0.85 \mathrm{~mm}$, and increased mean attachment gain by $0.3 \mathrm{~mm}$ in untreated chronic periodontitis patients.[152] Similar to Lopez's study, we noticed that CAL 
was less affected than PD with antibiotics use in BRONJ subjects, and bone height remained least affected. Resolving gingival inflammation alone with antibiotics use would decrease probe penetration and make CAL seem less severe. Antibiotics by themselves are expected to have least effect on alveolar bone height without additional interventions. A recent bacterial profiling study using 16s rDNA cultureindependent method suggests that oral antibiotic therapy have limited effect on the bacterial population associated with BRONJ lesions.[141] The study noted no significant differences in bacterial diversity among BRONJ patients treated with or without antibiotics. Parvimonas and Peptostreptococcus were more prevalent in antibiotic group, while Fusobacterium, Atopobium, and Streptococcus were predominant in non-antibiotic group indicating changes in the composition of biofilm between groups.

In the present study, all subjects were cancer patients receiving multiple chemotherapy regimens and were above the mean age of 60 years. Both old age and chemotherapy-induced immunosuppression are risk indicators for periodontal disease.[153,154] In consistence with the previous reports we noted number of BP infusions to be a significant risk factor for BRONJ development.[86,97,155] We also noted tooth extraction to be the common inciting factor for BRONJ development. This is in agreement with other published reports that have frequently noted BRONJ following extraction of un-restorable teeth including teeth with active periodontal disease or periapical infection.[85,110,120,121] In the absence of effective bone resorption, conditions like tooth extraction and dental infection might result in tissue 
death, vascular loss, and eventually osteonecrosis. Furthermore, we noted BRONJ subjects had more missing teeth than controls. It is unclear if BRONJ patients in the present study had lost their teeth due to periodontal disease, dental caries or other factors.

Recently, a few studies have noted that preventive dental measures initiated before the start of BP treatment reduce the incidence of BRONJ by 3 to 7 fold in multiple myeloma and bone metastasis patients.[156,157] In these studies, dental preventive measures included atraumatic tooth extraction, scaling/curettage, adjustment of ill fitting denture, dental restorations, and endodontic therapy. Furthermore, a retrospective study by Montefusco et al. noted that prophylactic antibiotics reduced the incidence of BRONJ lesions after dental procedures.[158] These findings reinforce the notion that poor oral hygiene and dental infection may play a role in BRONJ development. The American Dental Association recommends that patients on antiresorptive therapy who have active periodontal disease should receive appropriate forms of nonsurgical periodontal therapy combined with periodontal re-evaluation every four to six weeks.[159]'[92] The association further suggests that oral health program consisting of sound hygiene practices and regular dental care are valuable approaches for lowering risk of BRONJ development.[159][92] Since tooth extractions constitute a risk factor for BRONJ, patients are advised to undergo regular dental examination and be treated with the aim of preventing progression of periodontal disease to the point where dental extractions are necessary. Furthermore, 
when possible, practitioners are advised to use atraumatic techniques to minimize dentoalveolar manipulation.[159]'[92]

In conclusion, results from this cross-sectional study indicate that patients on intravenous BP therapy with poor periodontal status are at an increased risk for developing BRONJ. Antibiotics and chlorhexidine mouth rinse usage in BRONJ subjects may have eliminated or reduced the differences in clinical periodontal measurements between BRONJ cases and controls in the present study. Furthermore, it is unclear if discontinuation of BP therapy in BRONJ cases prior to the study enrolment may have affected our results. As a standard practice, patients are generally advised to discontinue BP treatment, and are prescribed antibiotics and chlorhexidine mouth rinse as soon as they are diagnosed with BRONJ. Hence, it is difficult to enroll BRONJ patients who are receiving BP therapy and who are not on antibiotics and chlorhexidine mouth rinse. Nevertheless, results from this preliminary study provide insight into the pathophysiology of BRONJ development and raise several hypotheses for future research. The causative role of poor oral hygiene in BRONJ development needs to be further explored in future prospective trials. 
Table 1. Baseline patient characteristics $(\mathrm{N}=73)$

\begin{tabular}{|c|c|c|c|}
\hline Characteristic & $\begin{array}{l}\text { BRONJ } \\
(\mathrm{N}=25)\end{array}$ & $\begin{array}{c}\text { Non-BRONJ } \\
(\mathrm{N}=48)\end{array}$ & P-value \\
\hline Age - mean (sd), in years & $66.3(10.3)$ & $64.4(11.7)$ & 0.50 \\
\hline $\begin{array}{l}\text { Sex }- \text { no. }(\%) \\
\text { Female }\end{array}$ & $16(64 \%)$ & $34(71 \%)$ & 0.60 \\
\hline $\begin{array}{l}\text { Race }- \text { no. }(\%) \\
\text { White } \\
\text { Black }\end{array}$ & $\begin{array}{c}24(96 \%) \\
1(4 \%)\end{array}$ & $\begin{array}{c}46(96 \%) \\
2(4 \%)\end{array}$ & 1.00 \\
\hline $\begin{array}{l}\text { Malignant Disease }- \text { no. }(\%) \\
\text { Breast cancer } \\
\text { Multiple myeloma } \\
\text { Prostate cancer } \\
\text { Lung cancer } \\
\text { Renal cell carcinoma } \\
\text { Others }\end{array}$ & $\begin{array}{l}13(52 \%) \\
4(16 \%) \\
2(8 \%) \\
2(8 \%) \\
3(12 \%) \\
1(4 \%)\end{array}$ & $\begin{array}{l}27(56 \%) \\
15(31 \%) \\
6(13 \%) \\
0(0 \%) \\
0(0 \%) \\
0(0 \%)\end{array}$ & 0.020 \\
\hline $\begin{array}{l}\text { Pre-existing medical conditions - no. (\%) } \\
\text { Diabetes mellitus } \\
\text { Hypertension } \\
\text { Hypercholesterolemia/Hyperlipidemia } \\
\text { Hypothyroidism } \\
\end{array}$ & $\begin{array}{c}1(4 \%) \\
11(44 \%) \\
11(44 \%) \\
7(28 \%) \\
\end{array}$ & $\begin{array}{l}5(10 \%) \\
26(54 \%) \\
15(33 \%) \\
10(21 \%) \\
\end{array}$ & $\begin{array}{l}0.66 \\
0.47 \\
0.45 \\
0.56\end{array}$ \\
\hline $\begin{array}{l}\text { Previous medications - no. }(\%) \\
\quad \text { Corticosteroids } \\
\end{array}$ & $14(56 \%)$ & $32(67 \%)$ & 0.45 \\
\hline $\begin{array}{l}\text { Smoking Status - no. }(\%) \\
\text { Current } \\
\text { Former } \\
\text { Never }\end{array}$ & $\begin{array}{c}5(20 \%) \\
11(44 \%) \\
9(36 \%) \\
\end{array}$ & $\begin{array}{c}3(6 \%) \\
18(38 \%) \\
27(56 \%)\end{array}$ & 0.11 \\
\hline $\begin{array}{l}\text { Type of Bisphosphonates }- \text { no. }(\%) \\
\text { Zoledronate } \\
\text { Pamidronate } \\
\text { Pamidronate }+ \text { Zoledronate }\end{array}$ & $\begin{array}{c}16(64 \%) \\
2(8 \%) \\
7(28 \%)\end{array}$ & $\begin{array}{l}32(67 \%) \\
6(13 \%) \\
10(21 \%)\end{array}$ & 0.71 \\
\hline $\begin{array}{l}\text { Bisphosphonate infusions - mean }(\mathrm{sd}) \\
\text { All cancer }\end{array}$ & $38.4(26.3)$ & $18.8(7.2)$ & $<0.0001^{*}$ \\
\hline $\begin{array}{l}\text { Duration of Bisphosphonate exposure - } \\
\text { mean (sd) in months } \\
\text { All cancer }\end{array}$ & $45.1(29.7)$ & $37.7(21.9)$ & 0.23 \\
\hline
\end{tabular}

$\S$ Pulsed corticosteroid treatment including dexamethasone or prednisone

$* \mathrm{P}=0.0011$ allowing unequal variances in the two groups 
Table 2. Unadjusted Comparisons of Clinical Periodontal Measurements (mean \pm SD)

\begin{tabular}{|l|c|c|c|}
\hline Characteristic & $\begin{array}{c}\text { BRONJ } \\
(\mathbf{N}=\mathbf{2 5})\end{array}$ & $\begin{array}{c}\text { Non-BRONJ } \\
(\mathbf{N = 4 8 )}\end{array}$ & P-value \\
\hline $\begin{array}{l}\text { Number of missing } \\
\text { teeth* }\end{array}$ & $9.7(9.4)$ & $3.1(4.6)$ & $0.0001^{* *}$ \\
\hline Mean Plaque Index & $0.40(0.29)$ & $0.55(0.41)$ & 0.13 \\
\hline Mean Gingival Index & $0.54(0.25)$ & $0.58(0.31)$ & 0.62 \\
\hline $\begin{array}{l}\text { Bleeding on Probing } \\
(\%)\end{array}$ & $12.0(15.4)$ & $14.9(12.8)$ & 0.42 \\
\hline Mean full-mouth PD (mm & $2.07(0.34)$ & $2.11(0.31)$ & 0.60 \\
\hline $\begin{array}{l}\text { Mean full-mouth CAL } \\
\text { (mm) }\end{array}$ & $2.18(1.55)$ & $1.56(0.99)$ & 0.047 \\
\hline \% Sites PD 4-6 mm & $5.0(4.6)$ & $4.8(6.5)$ & 0.87 \\
\hline \% Sites PD $>6 \mathrm{~mm}$ & $0.49(1.27)$ & $0.03(0.24)$ & $0.018^{* * *}$ \\
\hline \% Sites CAL $\geq 3 \mathrm{~mm}$ & $39.0(35.9)$ & $23.3(25.3)$ & 0.039 \\
\hline
\end{tabular}

* Out of 28 , i.e., not counting 3rd molars

** $\mathrm{P}=0.0026$ allowing different variances in the two groups $* * * \mathrm{P}=0.11$ allowing different variances in the two groups; $\mathrm{P}=0.005$ in Wilcoxon rank sum test. 
Table 3. Adjusted Comparisons ${ }^{\S}$ of Clinical Periodontal Measurements (mean $\pm \mathrm{SE}$ )

\begin{tabular}{|l|c|c|c|}
\hline Characteristic & $\begin{array}{c}\text { BRONJ } \\
\text { (N=25) }\end{array}$ & $\begin{array}{c}\text { Non- } \\
\text { BRONJ } \\
(\mathbf{N = 4 8 )}\end{array}$ & P value* \\
\hline Number of missing teeth** & $9.2(1.3)$ & $3.8(0.9)$ & 0.0008 \\
\hline Mean Plaque Index & $0.41(0.08)$ & $0.59(0.06)$ & 0.41 \\
\hline Mean Gingival Index & $0.53(0.06)$ & $0.59(0.05)$ & 0.99 \\
\hline Bleeding on P-valueing (\%) & $11.4(3.0)$ & $14.7(2.1)$ & 0.56 \\
\hline Mean full-mouth PD (mm) & $2.08(0.07)$ & $2.14(0.05)$ & 0.94 \\
\hline Mean full-mouth CAL (mm) & $2.13(0.22)$ & $1.71(0.16)$ & 0.10 \\
\hline \% Sites PD 4-6 mm & $4.7(1.3)$ & $5.2(0.9)$ & 0.49 \\
\hline \% Sites CAL $\geq 3 \mathrm{~mm}$ & $38.0(5.6)$ & $26.9(4.0)$ & 0.056 \\
\hline
\end{tabular}

${ }^{\S}$ Adjusted analyses are adjusted for age, sex, and smoking status (ever vs. never)

* Analyses including edentulous cases used multiple regression with ranktransformed outcome measurements as the dependent variables.

** Out of 28, i.e., not counting 3rd molars 
Table 4. BRONJ Characteristics $(\mathrm{N}=25)$

\begin{tabular}{|l|c|}
\hline Characteristics & BRONJ (N=25) \\
\hline Location - no. (\%) & $7(28 \%)^{*}$ \\
Maxilla & $20(80 \%)$ \\
Mandible & $4(16 \%)$ \\
Anterior sextant & $22(88 \%)$ \\
Posterior sextant & $16(64 \%)$ \\
\hline BRONJ onset ${ }^{\dagger}-$ no. (\%) & $6(24 \%)$ \\
Tooth extraction & $3(12 \%)$ \\
Spontaneous & $12(48 \%)$ \\
Others & $21(84 \%)$ \\
\hline BRONJ Treatment - no. $(\%)$ & \\
Antibiotics at enrolment & \\
Antimicrobial rinse at & \\
enrolment & \\
\hline
\end{tabular}

*Some subjects had BRONJ in more than one location

$\uparrow$ Other events precipitating BRONJ onset included implant placement and crown preparation. 
Table 5. Unadjusted Comparisons of Radiographic Alveolar Bone Height (means $\pm \mathrm{SD})$

\begin{tabular}{|l|c|c|c|}
\hline Characteristic & $\begin{array}{c}\text { BRONJ } \\
\mathbf{( N = 2 5 )}\end{array}$ & $\begin{array}{c}\text { Non- } \\
\text { BRONJ } \\
(\mathbf{N}=48)\end{array}$ & P-value \\
\hline Average bone height & $0.59(0.06)$ & $0.62(0.03)$ & $0.0041^{*}$ \\
\hline $\begin{array}{l}\text { Fraction teeth bone height } \\
\leq 0.50\end{array}$ & $0.20(0.23)$ & $0.06(0.09)$ & $0.0013 * *$ \\
\hline
\end{tabular}

$* \mathrm{P}=0.020$ allowing the two groups to have different variances

$* * \mathrm{P}=0.017$ allowing the two groups to have different variances 
Table 6. Adjusted Comparisons of Radiographic Alveolar Bone Height (means $\pm \mathrm{SE}$ )

\begin{tabular}{|l|c|c|c|}
\hline Characteristic & $\begin{array}{c}\text { BRONJ } \\
\mathbf{( N = 2 5 )}\end{array}$ & $\begin{array}{c}\text { Non- } \\
\text { BRONJ } \\
(\mathbf{N}=\mathbf{4 8})\end{array}$ & P value* \\
\hline Average bone height & $\begin{array}{c}0.596 \\
(0.008)\end{array}$ & $\begin{array}{c}0.624 \\
(0.006)\end{array}$ & 0.0029 \\
\hline $\begin{array}{l}\text { Fraction teeth bone height } \\
\leq 0.50\end{array}$ & $0.18(0.03)$ & $0.07(0.02)$ & 0.0009 \\
\hline
\end{tabular}

* Analyses including edentulous cases used multiple regression with ranktransformed outcome measurements as the dependent variables. 


\section{CHAPTER 4}

Salivary proteomics in Bisphosphonate-Related Osteonecrosis of the Jaw 


\section{Abstract}

Objective: A proteomic study was conducted to identify differentially expressed salivary proteins in "Bisphosphonate-Related Osteonecrosis of the Jaw (BRONJ)" patients that could serve as potential biomarkers for BRONJ diagnosis.

Materials and Methods: Whole saliva obtained from 20 BRONJ patients and 20 controls were pooled within groups according to BP exposure (for both BRONJ and control groups) and lesion size (BRONJ group only). The pooled samples were analyzed using iTRAQ labeled two-dimensional liquid chromatography-tandem mass spectrometry.

Results: Overall, 1340 proteins were identified. Of these, biomarker candidates were selected based on $p$ value $(<0.001)$, change in protein expression $(\geq 1.5$-fold increase or decrease relative to the respective comparison group), and unique peptides identified $(\geq 2)$. Three specific comparisons were made between BRONJ and control groups: (I) low-infusion controls versus low-infusion BRONJ; (II) high-infusion controls versus high-infusion BRONJ; and (III) high-infusion controls versus lowinfusion BRONJ. Two hundred proteins were found to be differentially expressed in BRONJ cases compared to controls, including 15 proteins in comparison-I, 78 in comparison-II, and 107 in comparison-III. The differentially expressed proteins were predicted to have a role in drug metabolism and molecular transport in comparison-I; cellular movement, hematological function and immune cell trafficking in comparison-II; and dermatological diseases, developmental disorder and organismal abnormalities in comparison-III. Of all the differentially expressed proteins, we selected two proteins for further validation. Immunoassays confirmed increased 
expression of metalloproteinase- 9 in individual saliva $(\mathrm{p}=0.048)$ and serum samples $(p=0.05)$ of BRONJ patients compared to controls. Desmoplakin was undetectable in saliva. However, their levels were decreased in BRONJ serum samples compared to controls, although the difference was statistically insignificant $(\mathrm{p}=0.157)$.

Conclusion: The molecular signatures identified by this cross-sectional study suggest that multiple pathological reactions are involved in BRONJ development. The role of MMP-9 and desmoplakin in BRONJ requires further investigation. 


\section{Introduction}

Long-term bisphosphonate (BP) therapy is associated with bisphosphonaterelated osteonecrosis of the jaw (BRONJ) [97,103]. To date, the true incidence, etiology and risk factors that contribute to BRONJ pathogenesis are largely unknown. Current diagnostic tools to assess skeletal health in patients receiving BP therapy are limited to imaging techniques, including bone scans and magnetic resonance imaging (MRI) [160]. These methods rely on changes in the physical characteristics of bone, which minimizes the likelihood of identifying "at risk" patients before they develop clinical signs of BRONJ. Therefore, it is important to identify biomarkers that precede the onset of BRONJ lesions. Patient-based proteomic studies are valuable approaches in discovering informative biomarkers for various diseases. [161]

In this study, we utilized an advanced quantitative proteomics approach to identify differentially expressed salivary proteins in BRONJ patients that can serve as potential biomarkers for BRONJ diagnosis.

\section{Material and Methods}

\section{Subjects}

The University of Minnesota Institutional Review Board approved this study and all participants provided written informed consent. Cancer patients above 30 years of age who had received intravenous BP therapy for cancer management were eligible for 
this study. Patients with a history of radiation therapy to the head and neck region or neoplasms (including metastasis) involving the head and neck region were excluded. The study population included 20 patients who developed BRONJ following intravenous BP therapy and 20 control patients who did not develop BRONJ following intravenous BP therapy. The American Association of Oral and Maxillofacial Surgeons criteria [85] was used to define BRONJ: “exposed bone in the maxillofacial area occurring in the absence of head and neck irradiation and showing no evidence of healing for at least 8 weeks after identification in patients treated with BP therapy".

\section{Saliva Sample Collection and Processing}

Unstimulated whole saliva $(5 \mathrm{~mL})$ was collected from BRONJ and control subjects by having each subject swallow and then expectorate continuously into $50-\mathrm{ml}$ sterile, polypropylene conical tube. Samples were centrifuged at 2,600g for 15 minutes at $4^{\circ} \mathrm{C}$. The supernatant containing the soluble fraction of salivary proteins was aliquoted into $1 \mathrm{~mL}$ tubes, mixed with protease inhibitors (courtesy David T. Wong, UCLA School of Dentistry, CA) and stored at $-80^{\circ} \mathrm{C}$.

\section{Protein Digestion, iTRAQ Labeling, and Peptide Fractionation}

Total protein concentration in the soluble fraction of saliva was quantified using the BCA assay (Thermo Pierce). Based on BRONJ lesion size, BRONJ subjects were 
characterized into "large lesion" $(\geq 10 \mathrm{~mm})$ and "small lesion" $(<10 \mathrm{~mm})$ groups. Notably, patients in the large lesion group had received higher number of BP infusions (mean=45) compared to small lesion BRONJ group (mean=37). Control subjects were categorized into "high" and "low" infusion groups based on whether they had received higher or lower infusions than the median number of infusion for all control patients (median=16). Pooled samples $(\mathrm{N}=10)$ were created for each of the four subgroups using $10 \mu \mathrm{g}$ of protein from each subject (Figure 1). Each pooled sample was digested overnight with trypsin according to filter-aided sample preparation (FASP) protocol [162]. Resulting peptides were concentrated and purified via reversed phase solid-phase extraction columns (Waters) and later labeled with the iTRAQ reagent (Applied Biosystems, Foster City) [163]. Subsequently, the iTRAQ labeled peptide mixtures were combined and fractionated using by strong cation exchange (SCX) chromatography [164]. Fractions were analyzed by reversed-phase microcapillary liquid chromatography [161].

\section{Mass Spectrometry}

Mass spectrometry was performed using a linear ion trap-Orbitrap (LTQ-Orbitrap) Velos instrument (Thermo Fisher Scientific) [165]. The LTQ-Orbitrap Velos was operated in a top-ten data dependent mode using survey scans at 30,000 resolution from 300 to $1800 \mathrm{~m} / \mathrm{z}$. Tandem MS scans were acquired with an isolation width of 2 $\mathrm{m} / \mathrm{z}$ and higher energy collisional dissociation (HCD) fragmentation mode with $40 \%$ normalized collision energy for 20 milliseconds. The automatic gain control settings 
were $3 \times 10^{5}$ ions in the ion trap, and $1 \times 10^{6}$ in the Orbitrap. Dynamic exclusion was used with duration of 15 seconds and a repeat count of 1 .

\section{Protein Identification and Quantification}

Raw files were converted to mzXml using msconvert (distributed as part of ProteoWizard 1.6.1260). Tandem mass spectra were searched against a human database including scrambled sequences and common contaminant proteins (136002 entries) using Sequestv27.0. Search parameters included a $1.6 \mathrm{amu}$ (atomic mass units) precursor and $0.8 \mathrm{amu}$ fragment mass tolerance, 2 missed cleavages, partial trypsin specificity, fixed modifications of cysteine acetamidylation and iTRAQ reagent at lysines and $\mathrm{N}$-termini, and variable modification of methionine oxidation. Search results were filtered to $99 \%$ protein probability and $95 \%$ peptide probability in Scaffold (v3.3.1, Proteome Software), producing false discovery rates of $0.8-3.6 \%$. Proteins were quantified using customized software developed by us [166] and biological meaning of differentially expressed proteins was assessed via bioinformatics analysis using the Ingenuity Pathway Analysis (IPA) software (Ingenuity Systems, Inc). All computational hardware and software was made available via an ongoing collaboration with the Minnesota Supercomputing Institute. 


\section{Statistical Analysis}

For mass spectrometry data, protein's abundance ratio and p-value were computed using open source software that implements an intensity-based weighted approach which is described elsewhere (Onsongo et al. 2010). For ELISA, differences between BRONJ and control groups were tested using a two-sided Student's t-test. P value below 0.05 was considered statistically significant.

\section{Results}

\section{Patient Characteristics}

Selected patient characteristics are described in Table 1. There were no significant differences in age $(\mathrm{P}=0.58)$, sex $(\mathrm{P}=0.27)$, or cancer diagnosis $(\mathrm{P}=0.42)$ between BRONJ cases and controls. The mean number of BP infusions was significantly higher in BRONJ cases compared to controls (41.0 \pm 27.2 infusions versus $17.9 \pm 8.1$; $\mathrm{P}<0.01$ ). BRONJ cases also received BP therapy for a longer period, (mean, 44.9 \pm 27.8 months versus $31.2 \pm 16.2$ months) although the difference was not significant $(\mathrm{P}=0.06)$. Most patients had received $\mathrm{BP}$ therapy for metastatic breast cancer management (12 BRONJ and 17 controls, Table 1).

Notably, at the time of saliva sampling, BRONJ cases had discontinued BP therapy for a mean period of 9.3 months (2 to 27 months) following initial BRONJ diagnosis, while controls continued to receive BP therapy for cancer management. 


\section{BRONJ Characteristics}

BRONJ was noted most frequently in the posterior region of the mandible $(75 \%)$. Most patients $(80 \%)$ developed BRONJ following dentoalveolar procedures, while some $(20 \%)$ developed it spontaneously without an obvious precipitating event. Pain was the common symptom in BRONJ cases with some exhibiting evidence of erythema and infection. As part of BRONJ management, most BRONJ cases were using antibiotics $(50 \%)$ or a chlorhexidine mouth rinse $(82 \%)$ at the time of study enrolment.

\section{Analysis of Salivary Proteomes}

Using proteomic profiling, we identified 1340 proteins. Of these proteins, biomarker candidates were selected based on $p$ value $(<0.001)$, the magnitude of change in protein expression (1.5-fold or greater increase or decrease) relative to the respective comparison group (e.g., comparing large lesion cases versus high infusion controls), and unique peptides identified (two or more).

Three specific comparisons were made between BRONJ and control groups: (I) lowinfusion controls versus low-infusion BRONJ; (II) high-infusion controls versus highinfusion BRONJ; and (III) high-infusion controls versus low-infusion BRONJ. Two hundred proteins were found to be differentially expressed in BRONJ cases compared to controls, including 15 proteins in comparison-I, 78 in comparison-II, and 107 in comparison-III. Table 2 lists the results from comparisons I and II. Furthermore, the number of BP infusions differed substantially between BRONJ cases and controls. In 
order to have a proximate comparison between cases and controls based on number of BP infusions, we analyzed high-infusion controls versus low-infusion BRONJ in comparison III. The results of this comparison are listed in Table 3.

The differentially expressed proteins in BRONJ subjects were predicted to have a role in drug metabolism and molecular transport in comparison-I; cellular movement, hematological function and immune cell trafficking in comparison-II; and dermatological diseases, developmental disorder and organismal abnormalities in comparison-III.

\section{Bioinformatics for Prioritizing and Selecting Biomarker Candidates}

Since validating all the identified proteins is time-consuming and expensive, we sought to prioritize the 92 proteins that were significantly differentially expressed in BRONJ cases compared to controls. Towards this end, we used IPA tool to group differentially expressed proteins into functional pathways. We postulated that such grouping would reveal proteins with possible ties to BRONJ development. The most significantly represented pathways were predicted to have a role in dermatological diseases and conditions, genetic disorders, inflammatory response, cancer, and immunological diseases. Among the prioritized proteins, we selected matrix metalloproteinase-9 (MMP-9) and desmoplakin as candidates for further validation. Our previous study indicates that MMP-9 is up-regulated in tooth extraction sockets of BP treated rats [167]. Also, it has been shown that BP treatment affects oral 
mucosa and delays epithelial wound healing in in vitro and in vivo models [142]. Therefore, we sought to validate desmoplakin, the most abundant desmosomal protein that is essential for maintaining the structural integrity of the epithelium.

\section{Validation of Biomarker Candidates by Immunoassays}

Enzyme-Linked Immunosorbent Assay (ELISA) was performed to detect the levels of MMP-9 (Quantikine, R\&D Systems, Minneapolis, MN) and desmoplakin (Blue Gene, Life Sciences Advanced Technologies Inc, Saint Petersburg, FL) in individual saliva $(n=40)$ and serum samples $(n=40)$ according to the manufacturer's instructions. We sought to analyze serum samples because true biological changes in protein expression will also be manifested in other biofluids apart from saliva. Similar to 2DLC-MS, immunoassays confirmed the increased expression of MMP-9 in saliva (mean $\pm \mathrm{SD}, 635 \pm 742$ vs $280 \pm 227 \mathrm{ng} / \mathrm{mL}, \mathrm{p}=0.048$ ) and serum samples (426 $\pm 386 \mathrm{vs}$ $246 \pm 161 \mathrm{ng} / \mathrm{mL}, \mathrm{p}=0.05)$ of BRONJ subjects. Desmoplakin levels were decreased in BRONJ serum samples, although it was not statistically significant (1.9 \pm 2.0 vs 2.8 $\pm 2.0 \mathrm{ng} / \mathrm{mL}, \mathrm{p}=0.157)$. Desmoplakin expression couldn't be detected in saliva samples; a possible explanation is that the primary antibody was not sensitive enough to detect desmoplakin expression in saliva. Nevertheless, desmoplakin serum result has a similar trend to that of salivary results obtained through 2D-LC-MS. 


\section{Discussion}

To date, no validated biomarker exists for BRONJ. With the increasing use of BP's by millions of patients worldwide, there is a critical need to identify at "risk patients" before they develop clinical manifestations of BRONJ. This is the first study to identify differentially expressed salivary proteins in BRONJ patients that could serve as potential biomarkers for BRONJ diagnosis. Using a comprehensive and rigorous proteomic analysis, we identified 92 proteins that were significantly differentially expressed in BRONJ cases compared to controls. Many of the identified proteins require further scrutiny. As a preliminary step, we sought to examine MMP-9 and desmoplakin in the present study and found MMP-9 to be increased and desmoplakin to be decreased in BRONJ cases compared to controls.

Matrix metalloproteinase-9 (MMP-9): Is a key proteolytic enzyme involved in wound healing [168,169], and is essential for normal bone development and remodeling [170]. MMP-9 degrades collagen fibrils, basement membranes, and other supra-structures of the extracellular matrix [171,172]. It is primarily expressed by osteoclasts, but is also expressed by macrophages and fibroblasts during wound healing process $[173,174]$.

Several studies have evaluated the effects of BP on Matrix metalloproteinases (MMPs) expression in cancer cells $[175,176]$. In premalignant and malignant conditions, BP's inhibit angiogenesis by targeting both macrophage expression and proteolytic activity of MMPs [175]. However, the effects of BP on MMPs from the 
normal oral mucosal cells are unclear. Allam et al. noted a significant reduction in MMP-9 expression in oral epithelial cells following zoledronate treatment [177]. In the present study, we noted increased MMP-9 expression in BRONJ cases. The reason for elevated MMP-9 expression in BRONJ cases is unclear. One possibility is that there is a rapid rebound in the number of osteoclasts and osteoclast activity after BPs are discontinued in cancer patients. In this study, BRONJ cases had discontinued BP therapy for at least 2 months and a mean of 9.3 months. Controls were undergoing BP treatment at the time of study enrolment. In support of the current findings, in our previous animal study, we noted that BP-treated rats had increased MMP-9 mRNA, protein expression and enzymatic activity during tooth extraction wound healing (Basi et al. 2011).

Overall, the increased MMP-9 expression in BRONJ cases could play a vital role in BRONJ healing by facilitating keratinocyte migration and granulation tissue remodeling $[168,169]$.

Desmosomal proteins: Desmosomes are intercellular adhesive junctions that link epithelial cells to each other and attach keratin intermediate filament to the cell surface. This assembly not only allows epithelial tissues to withstand mechanical stress, but also facilitates cell-cell communication through signal transmission. Any disruption in the desmosome-keratin filament complex results in a breakdown in cell adhesion leading to various skin, hair, and heart defects [178]. 
Several genetic and autoimmune diseases involving desmosomal proteins such as desmoplakin have been described. Mutations that truncate the desmoplakin Cterminus results in a lethal condition termed acantholytic epidermolysis bullosa [179]. Patients with skin fragility-ectodermal dysplasia syndrome have mutations in plakophilin-1 [180] and exhibit severe acantholysis (loss of cell-cell adhesion), which highlights the importance of plakophilins in desmosomal adhesion. Autoantibodies to periplakin and envoplakin, members of the plakin family with structures similar to desmoplakin, are present in the mucocutaneous blistering-disease paraneoplastic pemphigus [181]. Furthermore, plakoglobin together with plakophilin-1 and 3, play important role in facilitating the adhesion of desmoplakin to keratin intermediate filaments and in mediating important signal transduction pathways [182]. Plakoglobin-knockout mice exhibit skin blistering from acantholysis, indicating that plakoglobins are essential in maintaining the structural integrity of the epidermis [183].

Collectively, these mutations provide insight into the functional domains of desmoplakin proteins and their relevance to skin biology. In the present study, 2DLC-MS analysis revealed desmoplakin, periplakin, envoplakin, junction plakoglobin, and plakophilin-1 expressions were significantly downregulated in BRONJ cases compared to controls (Table 3). Immunoassay performed on individual serum samples confirmed decreased expression of desmoplakin in BRONJ subjects, although the difference was statistically insignificant $(\mathrm{p}=0.157)$. It is unclear if $\mathrm{BP}$ therapy affects desmosomal proteins, or if patients with desmosomal protein 
insufficiency are at a higher risk of developing BRONJ. Previously published reports have noted that $20 \%$ of the BRONJ lesions occur spontaneously without an inciting event. It is possible that impairment in desmosomal proteins could lead to a compromise in the structural integrity of the epithelium leading to spontaneous BRONJ development.

Furthermore, using proteomics approaches, we found keratins to be significantly downregulated in BRONJ cases compared to controls. Previous studies have demonstrated that BP's suppress cellular proliferation and delay wound healing of oral keratinocytes [184]. Mawardi et al., in a murine model, showed that the combination of pamidronate and Fusobacterium nucleatum caused BRONJ-like lesions and delayed epithelial wound healing as result of diminished production of keratinocyte growth factor by gingival fibroblasts [142]. In an in vitro model, Kim et al. demonstrated that pamidronate induced senescence in normal human oral keratinocytes and impaired re-epithelialization of oral mucosa [185]. Altogether, these findings suggest that impairment in cell-junction-related proteins may play a vital role in BRONJ development and affect BRONJ healing process. It is possible that higher concentrations of BP's in jawbones may cause direct toxicity to the oral epithelium affecting cell-junction-related proteins.

This cross-sectional study has several limitations. Despite attempts to match cases and controls according to $\mathrm{BP}$ exposure, the number of $\mathrm{BP}$ infusions differed significantly between BRONJ cases and controls. The disparity in BP treatment 
between the groups could explain some of the differences in protein expression between BRONJ subjects and controls. Also, the discontinuation of BP therapy in BRONJ cases prior to the study enrolment may have affected our results. Because patients are generally advised to discontinue BP treatment as soon as they are diagnosed with BRONJ, it is difficult to enroll BRONJ patients who are receiving BP therapy. However, results from this preliminary study provide insight into the pathophysiology of BRONJ development and raise several hypotheses for future research. One or more of the differentially expressed proteins identified by this study may prove to be useful biomarkers for BRONJ diagnosis. The role of cell-junctionrelated proteins in BRONJ development needs to be further investigated. 
Figure 1. Biomarker Discovery Method

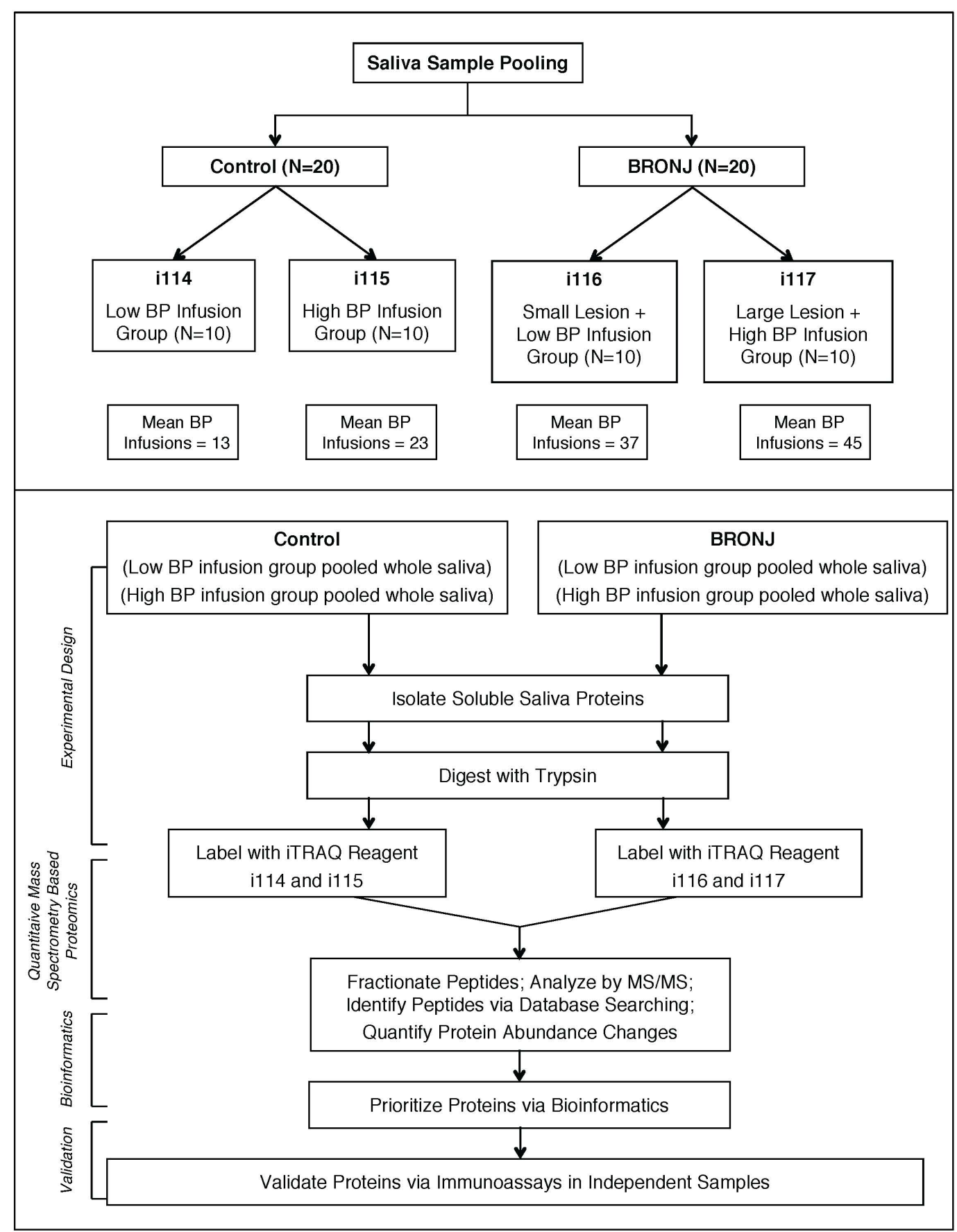


Table 1. Baseline patient characteristics $(\mathrm{N}=40)$

\begin{tabular}{|llll|}
\hline Characteristic & $\begin{array}{l}\text { BRONJ } \\
(\mathbf{N}=\mathbf{2 0})\end{array}$ & $\begin{array}{l}\text { Non-BRONJ } \\
(\mathbf{N}=\mathbf{2 0})\end{array}$ & $\boldsymbol{P} \boldsymbol{}$ \\
Age - years & $64.3 \pm 10.1$ & $62.4 \pm 11.3$ & 0.58 \\
Sex - no. $(\%)$ & & & 0.27 \\
$\quad$ Male & $7(35)$ & $3(15)$ & \\
$\quad$ Female & $13(65)$ & $17(85)$ & 0.42 \\
Malignant Disease - no. $(\%)$ & $12(60)$ & $17(85)$ & \\
$\quad$ Breast cancer & $2(10)$ & $2(10)$ & \\
$\quad$ Multiple myeloma & $1(5)$ & $1(5)$ & \\
$\quad$ Prostate cancer & $2(10)$ & $0(0)$ & \\
$\quad$ Lung cancer & $2(10)$ & $0(0)$ & \\
$\quad$ Renal cell carcinoma & $1(5)$ & $0(0)$ & \\
$\quad$ Others & & & \\
Type of Bisphosphonates - no. $(\%)$ & $14(70)$ & $18(90)$ & \\
$\quad$ Zoledronate & $2(10)$ & $0(0)$ & \\
$\quad$ Pamidronate & $4(20)$ & $2(10)$ & \\
$\quad$ Pamidronate + Zoledronate & & & \\
Bisphosphonate infusions & & $17.9 \pm 8.1$ & \\
All cancer & $41.0 \pm 27.2$ & & \\
Duration of Bisphosphonate exposure & & $31.2 \pm 16.2$ & \\
- in months & $44.9 \pm 27.8$ & & \\
$\quad$ All cancer & & & \\
& & & \\
\end{tabular}

- Plus-minus values are means $\pm \mathrm{SD}$

- $\quad$ P-values were calculated from a Fisher's-exact test or a t-test for categorical or continuous variables, respectively. 
Table 2. Differentially expressed salivary proteins among BRONJ cases in both the high BP infusion and low BP infusion groups, collectively

\begin{tabular}{|c|c|c|c|c|}
\hline $\begin{array}{l}\text { Accession } \\
\text { Number }\end{array}$ & $\begin{array}{c}\text { Unique } \\
\text { Peptides }\end{array}$ & Protein Name & $\begin{array}{l}\text { Fold } \\
\text { Change }^{\dagger}\end{array}$ & $\mathrm{P}$ value \\
\hline \multicolumn{5}{|c|}{ High BP Infusion Group: i117 vs i115 (BRONJ vs Controls) } \\
\hline Q53FA3 & 2 & Heat shock 70kDa protein 1-like & $\Uparrow 4.099$ & $8.65 \mathrm{E}-05$ \\
\hline C9JRG0 & 2 & Hemoglobin, delta & $\Uparrow 3.206$ & $6.24 \mathrm{E}-07$ \\
\hline F5H3W7 & 20 & Matrix metallopeptidase 9 & $\Uparrow 2.903$ & $1.95 \mathrm{E}-04$ \\
\hline E7EW61 & 5 & Transthyretin & $\Uparrow 2.870$ & $3.09 \mathrm{E}-10$ \\
\hline $\mathrm{P} 02763$ & 9 & Alpha-1-acid glycoprotein 1 & $\Uparrow 2.848$ & $1.90 \mathrm{E}-05$ \\
\hline P14780 & 2 & Matrix metalloproteinase- 9 & $\Uparrow 2.806$ & $5.48 \mathrm{E}-09$ \\
\hline P01857 & 23 & Ig gamma-1 chain $\mathrm{C}$ region & $\Uparrow 2.715$ & $3.36 \mathrm{E}-04$ \\
\hline P80419 & 2 & Ig heavy chain V-III region GAR & $\Uparrow 2.656$ & $1.98 \mathrm{E}-05$ \\
\hline P05546 & 4 & Heparin cofactor 2 & $\Uparrow 2.601$ & $2.97 \mathrm{E}-13$ \\
\hline P01009-2 & 3 & Isoform 2 of Alpha-1-antitrypsin & $\Uparrow 2.600$ & $6.78 \mathrm{E}-14$ \\
\hline O15144 & 2 & Actin-related protein $2 / 3$ complex subunit 2 & $\Uparrow 2.361$ & $1.39 \mathrm{E}-14$ \\
\hline P29622 & 3 & Kallistatin & $\Uparrow 2.335$ & $3.56 \mathrm{E}-04$ \\
\hline $\mathrm{P} 02652$ & 6 & Apolipoprotein A-II & $\Uparrow 2.285$ & $1.90 \mathrm{E}-05$ \\
\hline Q9H9S4 & 2 & Calcium-binding protein 39-like & $\Uparrow 2.242$ & $2.96 \mathrm{E}-10$ \\
\hline P01880 & 2 & Ig delta chain $\mathrm{C}$ region & $\Uparrow 2.148$ & $2.89 \mathrm{E}-15$ \\
\hline P63267 & 3 & Actin, gamma-enteric smooth muscle & $\Uparrow 2.115$ & $4.13 \mathrm{E}-07$ \\
\hline P01031 & 4 & Complement C5 & $\Uparrow 2.104$ & $1.44 \mathrm{E}-15$ \\
\hline P35542 & 2 & Serum amyloid A-4 protein & $\Uparrow 2.102$ & $7.91 \mathrm{E}-04$ \\
\hline $\mathrm{P} 04217$ & 4 & Alpha-1B-glycoprotein & $\Uparrow 2.062$ & 4.24E-04 \\
\hline P61586 & 2 & Transforming protein RhoA & $\Uparrow 2.039$ & $6.90 \mathrm{E}-09$ \\
\hline P01763 & 2 & Ig heavy chain V-III region WEA & $\Uparrow 2.034$ & $2.16 \mathrm{E}-07$ \\
\hline Q9NUQ9 & 6 & Protein FAM49B & $\Uparrow 2.002$ & $3.98 \mathrm{E}-05$ \\
\hline Q5W0X3 & 2 & FK506 binding protein $1 \mathrm{~A}, 12 \mathrm{kDa}$ & $\Uparrow 1.964$ & $8.65 \mathrm{E}-06$ \\
\hline Q15782 & 6 & Chitinase-3-like protein 2 & $\Uparrow 1.929$ & $2.78 \mathrm{E}-05$ \\
\hline P35241 & 3 & Radixin & $\Uparrow 1.878$ & $9.46 \mathrm{E}-13$ \\
\hline P62745 & 2 & Rho-related GTP-binding protein RhoB & $\Uparrow 1.826$ & $2.11 \mathrm{E}-10$ \\
\hline D6RHI9 & 3 & Ribonuclease T2 & $\Uparrow 1.812$ & $4.50 \mathrm{E}-07$ \\
\hline $\mathrm{P} 01770$ & 2 & Ig heavy chain V-III region NIE & $\Uparrow 1.766$ & $5.58 \mathrm{E}-04$ \\
\hline P06316 & 2 & Ig lambda chain V-I region BL2 & $\Uparrow 1.756$ & $1.17 \mathrm{E}-04$ \\
\hline P01776 & 2 & Ig heavy chain V-III region WAS & $\Uparrow 1.738$ & $2.77 \mathrm{E}-04$ \\
\hline P00736 & 2 & Complement C1r subcomponent & $\Uparrow 1.721$ & $9.64 \mathrm{E}-12$ \\
\hline B4DJS1 & 3 & cDNA FLJ57383, highly similar to Coronin-1A & $\Uparrow 1.698$ & $1.48 \mathrm{E}-06$ \\
\hline C9JZD1 & 2 & Actin related protein $2 / 3$ complex, subunit $3,21 \mathrm{kDa}$ & $\Uparrow 1.628$ & $3.61 \mathrm{E}-04$ \\
\hline O60888-2 & 2 & Isoform A of Protein CutA & $\Uparrow 1.620$ & $8.97 \mathrm{E}-07$ \\
\hline $\operatorname{tr}$ & 2 & Uncharacterized protein $\mathrm{OS}=$ Homo sapiens $\mathrm{PE}=4 \mathrm{SV}=1$ & $\Uparrow 1.585$ & $7.09 \mathrm{E}-04$ \\
\hline P04430 & 3 & Ig kappa chain V-I region BAN & $\Uparrow 1.570$ & $3.56 \mathrm{E}-04$ \\
\hline P19823 & 4 & Inter-alpha-trypsin inhibitor heavy chain $\mathrm{H} 2$ & $\Uparrow 1.561$ & $9.33 \mathrm{E}-05$ \\
\hline B4DTM7 & 6 & cDNA FLJ53006, highly similar to Vinculin & $\Uparrow 1.555$ & $1.08 \mathrm{E}-04$ \\
\hline P0CG05 & 2 & Ig lambda- 2 chain $\mathrm{C}$ regions & $\Uparrow 1.546$ & $5.51 \mathrm{E}-06$ \\
\hline Q5JZH0 & 2 & Cathepsin A & $\Uparrow 1.544$ & 4.04E-07 \\
\hline F6TTL5 & 2 & Actin-related protein $2 / 3$ complex subunit 4 & $\Uparrow 1.521$ & $4.44 \mathrm{E}-16$ \\
\hline Q86Y46 & 2 & Keratin, type II cytoskeletal 73 & $\Downarrow 31.745$ & $1.46 \mathrm{E}-10$ \\
\hline P13647 & 2 & Keratin, type II cytoskeletal 5 & $\Downarrow 18.968$ & $2.87 \mathrm{E}-13$ \\
\hline C9JKY1 & 11 & Junction plakoglobin & $\Downarrow 10.649$ & $3.68 \mathrm{E}-05$ \\
\hline O75556 & 4 & Mammaglobin-B & $\Downarrow 7.969$ & $3.65 \mathrm{E}-05$ \\
\hline Q5T3N1 & 8 & Annexin A1 & $\Downarrow 7.875$ & $1.85 \mathrm{E}-04$ \\
\hline $\mathrm{P} 04792$ & 8 & Heat shock protein beta-1 & $\Downarrow 6.669$ & $2.12 \mathrm{E}-04$ \\
\hline O95171 & 7 & Sciellin & $\Downarrow 6.573$ & $1.32 \mathrm{E}-12$ \\
\hline Q7Z3Z0 & 2 & Keratin, type I cytoskeletal 25 & $\Downarrow 6.165$ & $1.99 \mathrm{E}-04$ \\
\hline
\end{tabular}




\begin{tabular}{|c|c|c|c|c|}
\hline $\mathrm{P} 02545$ & 7 & Lamin A/C & $\Downarrow 5.924$ & $1.86 \mathrm{E}-04$ \\
\hline Q6ZN66 & 2 & Guanylate-binding protein 6 & $\Downarrow 5.722$ & $7.44 \mathrm{E}-08$ \\
\hline Q96FQ6 & 3 & Protein S100-A16 & $\Downarrow 5.472$ & $5.30 \mathrm{E}-11$ \\
\hline $\mathrm{P} 22735$ & 8 & Protein-glutamine gamma-glutamyltransferase K & $\Downarrow 4.374$ & $9.85 \mathrm{E}-04$ \\
\hline P62851 & 3 & 40S ribosomal protein $\mathrm{S} 25$ & $\Downarrow 4.158$ & $3.64 \mathrm{E}-10$ \\
\hline B4DJ43 & 3 & cDNA FLJ53341, highly similar to Tubulin beta-4 chain & $\Downarrow 3.325$ & $1.11 \mathrm{E}-15$ \\
\hline $\mathrm{P} 25705$ & 3 & ATP synthase subunit alpha, mitochondrial & $\Downarrow 3.091$ & $1.32 \mathrm{E}-06$ \\
\hline Q5SQY1 & 3 & Novel protein similar to beta-tubulin 4Q & $\Downarrow 3.070$ & $3.09 \mathrm{E}-05$ \\
\hline P63241-2 & 3 & Isoform 2 of Eukaryotic translation initiation factor $5 \mathrm{~A}-1$ & $\Downarrow 3.034$ & $9.12 \mathrm{E}-04$ \\
\hline $\mathrm{P} 25398$ & 4 & 40S ribosomal protein $\mathrm{S} 12$ & $\Downarrow 2.833$ & $5.79 \mathrm{E}-06$ \\
\hline P68104 & 3 & Elongation factor 1-alpha 1 & $\Downarrow 2.801$ & $1.06 \mathrm{E}-07$ \\
\hline P61026 & 3 & Ras-related protein Rab-10 & $\Downarrow 2.731$ & $1.08 \mathrm{E}-05$ \\
\hline O60218 & 6 & Aldo-keto reductase family 1 member B10 & $\Downarrow 2.605$ & $9.68 \mathrm{E}-06$ \\
\hline B4DPU3 & 4 & cDNA FLJ56548, highly similar to Elongation factor 2 & $\Downarrow 2.566$ & $4.55 \mathrm{E}-12$ \\
\hline $\mathrm{A} 8 \mathrm{~K} 2 \mathrm{U} 0$ & 11 & Alpha-2-macroglobulin-like protein 1 & $\Downarrow 2.522$ & $3.61 \mathrm{E}-04$ \\
\hline Q9NZT1 & 7 & Calmodulin-like protein 5 & $\Downarrow 2.488$ & $6.82 \mathrm{E}-04$ \\
\hline P07951 & 3 & Tropomyosin 2 (Beta) & $\Downarrow 2.314$ & 7.67E-04 \\
\hline P30838 & 2 & Aldehyde dehydrogenase, dimeric NADP-preferring & $\Downarrow 2.049$ & $1.40 \mathrm{E}-10$ \\
\hline B4DLA9 & 2 & Histone $\mathrm{H} 2 \mathrm{~B}$ & $\Downarrow 2.018$ & $1.64 \mathrm{E}-13$ \\
\hline P29373 & 2 & Cellular retinoic acid binding protein 2 & $\Downarrow 1.997$ & $3.27 \mathrm{E}-09$ \\
\hline Q6LES2 & 4 & Annexin & $\Downarrow 1.984$ & $4.06 \mathrm{E}-04$ \\
\hline Q5JR95 & 2 & Ribosomal protein S8 & $\Downarrow 1.978$ & $2.93 \mathrm{E}-04$ \\
\hline ALDH3A1 & 4 & ldehyde dehydrogenase, dimeric NADP-preferring & $\Downarrow 1.940$ & $1.36 \mathrm{E}-10$ \\
\hline P05120 & 3 & Plasminogen activator inhibitor 2 & $\Downarrow 1.894$ & $2.05 \mathrm{E}-10$ \\
\hline Q9Y2V2 & 2 & Calcium-regulated heat stable protein 1 & $\Downarrow 1.792$ & $1.36 \mathrm{E}-11$ \\
\hline P50990 & 3 & T-complex protein 1 subunit theta & $\Downarrow 1.729$ & $1.91 \mathrm{E}-10$ \\
\hline P08493 & 2 & Matrix Gla protein & $\Downarrow 1.699$ & $3.08 \mathrm{E}-08$ \\
\hline O75390 & 3 & Citrate synthase, mitochondrial & $\Downarrow 1.516$ & $2.06 \mathrm{E}-07$ \\
\hline \multicolumn{5}{|c|}{ Low BP Infusion Group: i116 vs i114 (BRONJ vs Controls) } \\
\hline $\mathrm{O} 75556$ & 4 & Mammaglobin-B & $\Uparrow 13.885$ & $8.71 \mathrm{E}-08$ \\
\hline C9JRG0 & 2 & Hemoglobin, delta & $\Uparrow 7.914$ & $1.16 \mathrm{E}-06$ \\
\hline P69892 & 2 & Hemoglobin subunit gamma-2 & $\Uparrow 5.308$ & $5.91 \mathrm{E}-04$ \\
\hline Q04118 & 3 & Basic salivary proline-rich protein 3 & $\Uparrow 2.408$ & $3.54 \mathrm{E}-04$ \\
\hline P00918 & 4 & Carbonic anhydrase 2 & $\Uparrow 2.230$ & $6.84 \mathrm{E}-06$ \\
\hline P34096 & 2 & Ribonuclease 4 & $\Uparrow 1.722$ & $7.56 \mathrm{E}-08$ \\
\hline P80723 & 5 & Brain acid soluble protein 1 & $\Uparrow 1.660$ & $6.59 \mathrm{E}-04$ \\
\hline $\mathrm{P} 25705$ & 3 & ATP synthase subunit alpha, mitochondrial & $\Uparrow 1.547$ & $1.35 \mathrm{E}-04$ \\
\hline $\mathrm{P} 05546$ & 4 & Heparin cofactor 2 & $\Uparrow 1.502$ & $8.06 \mathrm{E}-09$ \\
\hline C9JMC5 & 4 & Aldehyde dehydrogenase 3 family, member A1 & $\Downarrow 2.310$ & $3.11 \mathrm{E}-15$ \\
\hline P09211 & 2 & Glutathione S-transferase P & $\Downarrow 2.123$ & $5.46 \mathrm{E}-07$ \\
\hline O60218 & 6 & Aldo-keto reductase family 1 member B10 & $\Downarrow 1.850$ & $2.41 \mathrm{E}-09$ \\
\hline P55786 & 4 & Puromycin-sensitive aminopeptidase & $\Downarrow 1.622$ & $1.29 \mathrm{E}-06$ \\
\hline $\mathrm{P} 06731$ & 2 & $\begin{array}{l}\text { Carcinoembryonic antigen-related cell adhesion } \\
\text { molecule } 5\end{array}$ & $\Downarrow 1.572$ & 3.73E-04 \\
\hline Q5W0H4 & 2 & Tumor protein, translationally-controlled 1 & $\Downarrow 1.557$ & $7.70 \mathrm{E}-04$ \\
\hline
\end{tabular}

\section{$\uparrow \Uparrow$ Represents upregulation and $\Downarrow$ Represents downregulation}


Table 3. Differentially expressed salivary proteins in low BP infusion BRONJ group compared to high BP infusion control group

\begin{tabular}{|c|c|c|c|c|}
\hline $\begin{array}{l}\text { Accession } \\
\text { Number }\end{array}$ & $\begin{array}{c}\text { Unique } \\
\text { Peptides }\end{array}$ & Protein Name & $\begin{array}{l}\text { Fold } \\
\text { Change }^{\dagger}\end{array}$ & $\mathrm{P}$ value \\
\hline \multicolumn{5}{|c|}{ Low BP Infusion BRONJ Group (i116) vs High BP Infusion Control Group (i115) } \\
\hline Q86Y46 & 2 & Keratin, type II cytoskeletal 73 & $\Downarrow 6.554$ & $2.11 \mathrm{E}-15$ \\
\hline P02538 & 28 & Keratin, type II cytoskeletal $6 \mathrm{~A}$ & $\Downarrow 6.022$ & $1.02 \mathrm{E}-07$ \\
\hline P13646 & 50 & Keratin, type I cytoskeletal 13 & $\Downarrow 5.558$ & $6.80 \mathrm{E}-07$ \\
\hline P12035 & 10 & Keratin, type II cytoskeletal 3 & $\Downarrow 5.302$ & $1.20 \mathrm{E}-07$ \\
\hline B4DRW1 & 40 & $\begin{array}{l}\text { DNA FLJ } 55805 \text {, highly similar to Keratin, type II } \\
\text { cytoskeletal } 4\end{array}$ & $\Downarrow 5.222$ & $3.23 \mathrm{E}-05$ \\
\hline P14923 & 11 & Junction plakoglobin & $\Downarrow 5.001$ & $3.44 \mathrm{E}-15$ \\
\hline P08727 & 8 & Keratin, type I cytoskeletal 19 & $\Downarrow 4.861$ & $2.94 \mathrm{E}-05$ \\
\hline Q01546 & 11 & Keratin, type II cytoskeletal 2 oral & $\Downarrow 4.850$ & $1.60 \mathrm{E}-06$ \\
\hline P08779 & 40 & Keratin, type I cytoskeletal 16 & $\Downarrow 4.632$ & $8.83 \mathrm{E}-05$ \\
\hline P47929 & 6 & Galectin-7 & $\Downarrow 4.624$ & $8.57 \mathrm{E}-06$ \\
\hline P19012 & 2 & Keratin, type I cytoskeletal 15 & $\Downarrow 4.453$ & $1.55 \mathrm{E}-15$ \\
\hline Q5T3N1 & 8 & Annexin & $\Downarrow 4.438$ & $3.56 \mathrm{E}-07$ \\
\hline $\mathrm{P} 07355$ & 8 & Annexin A2 & $\Downarrow 4.307$ & $7.90 \mathrm{E}-08$ \\
\hline P13647 & 11 & Keratin, type II cytoskeletal 5 & $\Downarrow 4.090$ & 4.62E-04 \\
\hline Q9HCY8 & 6 & Protein S100-A14 & $\Downarrow 4.050$ & $7.72 \mathrm{E}-07$ \\
\hline Q8N1N4 & 14 & Keratin, type II cytoskeletal 78 & $\Downarrow 4.035$ & $3.74 \mathrm{E}-05$ \\
\hline P15924 & 43 & Desmoplakin & $\Downarrow 3.942$ & $1.38 \mathrm{E}-04$ \\
\hline $\mathrm{P} 02533$ & 10 & Keratin, type I cytoskeletal 14 & $\Downarrow 3.803$ & $9.50 \mathrm{E}-04$ \\
\hline Q7Z3Z0 & 2 & Keratin, type I cytoskeletal 25 & $\Downarrow 3.782$ & $2.11 \mathrm{E}-15$ \\
\hline Q13835 & 12 & Plakophilin-1 & $\Downarrow 3.751$ & $1.59 \mathrm{E}-05$ \\
\hline P04083 & 14 & Annexin A1 & $\Downarrow 3.544$ & $2.76 \mathrm{E}-04$ \\
\hline $\mathrm{P} 04792$ & 8 & Heat shock protein beta-1 & $\Downarrow 3.477$ & $2.77 \mathrm{E}-11$ \\
\hline P20930 & 2 & Filaggrin & $\Downarrow 3.431$ & $3.03 \mathrm{E}-14$ \\
\hline B4DLA9 & 2 & Histone H2B type 2-F & $\Downarrow 3.332$ & $2.89 \mathrm{E}-15$ \\
\hline P22735 & 8 & Protein-glutamine gamma-glutamyltransferase K & $\Downarrow 3.330$ & $1.99 \mathrm{E}-12$ \\
\hline O60437 & 39 & Periplakin & $\Downarrow 3.283$ & $3.70 \mathrm{E}-05$ \\
\hline Q92817 & 23 & Envoplakin & $\Downarrow 3.129$ & $3.82 \mathrm{E}-06$ \\
\hline Q6ZN66 & 9 & Guanylate-binding protein 6 & $\Downarrow 3.061$ & $2.48 \mathrm{E}-07$ \\
\hline P62851 & 3 & 40S ribosomal protein S25 & $\Downarrow 3.041$ & 3.99E-14 \\
\hline Q9UBG3 & 9 & Cornulin & $\Downarrow 3.029$ & $7.81 \mathrm{E}-05$ \\
\hline P19013 & 3 & Keratin, type II cytoskeletal 4 & $\Downarrow 2.821$ & $7.85 \mathrm{E}-04$ \\
\hline B4DQ53 & 6 & cDNA FLJ51275 & $\Downarrow 2.690$ & $2.16 \mathrm{E}-06$ \\
\hline B4DPU3 & 4 & cDNA FLJ56548, highly similar to Elongation factor 2 & $\Downarrow 2.642$ & $6.11 \mathrm{E}-15$ \\
\hline P05388 & 3 & $60 \mathrm{~S}$ acidic ribosomal protein $\mathrm{P} 0$ & $\Downarrow 2.618$ & $5.69 \mathrm{E}-06$ \\
\hline P63241 & 3 & Eukaryotic translation initiation factor 5A-1 & $\Downarrow 2.605$ & $3.89 \mathrm{E}-15$ \\
\hline Q96QV6 & 3 & Histone H2A type 1-A & $\Downarrow 2.580$ & $5.75 \mathrm{E}-12$ \\
\hline Q9H4B7 & 4 & Tubulin beta-1 chain & $\Downarrow 2.553$ & 3.97E-04 \\
\hline Q16778 & 9 & Histone H2B type 2-E & $\Downarrow 2.543$ & $8.52 \mathrm{E}-06$ \\
\hline Q5JR95 & 2 & 40S ribosomal protein $\mathrm{S} 8$ & $\Downarrow 2.530$ & $1.52 \mathrm{E}-06$ \\
\hline Q5SQY1 & 3 & Novel protein similar to beta-tubulin 4Q & $\Downarrow 2.472$ & $8.29 \mathrm{E}-14$ \\
\hline O75367 & 3 & Core histone macro-H2A.1 & $\Downarrow 2.405$ & $9.82 \mathrm{E}-08$ \\
\hline Q59GP5 & 3 & Eukaryotic translation elongation factor 1 alpha 2 variant & $\Downarrow 2.405$ & $6.65 \mathrm{E}-05$ \\
\hline P01040 & 8 & Cystatin-A & $\Downarrow 2.321$ & $1.97 \mathrm{E}-05$ \\
\hline P08238 & 17 & Heat shock protein HSP 90-beta & $\Downarrow 2.250$ & $6.54 \mathrm{E}-10$ \\
\hline P31947 & 18 & Epithelial cell marker protein 1 & $\Downarrow 2.240$ & $6.99 \mathrm{E}-15$ \\
\hline P16401 & 4 & Histone H1.5 & $\Downarrow 2.193$ & $4.39 \mathrm{E}-08$ \\
\hline Q16695 & 3 & Histone $\mathrm{H} 3.1 \mathrm{t}$ & $\Downarrow 2.181$ & $6.89 \mathrm{E}-08$ \\
\hline
\end{tabular}




\begin{tabular}{|c|c|c|c|c|}
\hline $\mathrm{P} 15515$ & 6 & Histatin-1 & $\Downarrow 2.158$ & $5.53 \mathrm{E}-05$ \\
\hline P14555 & 3 & Phospholipase A2, membrane associated & $\Downarrow 2.134$ & $1.43 \mathrm{E}-14$ \\
\hline P50990 & 3 & T-complex protein 1 subunit theta & $\Downarrow 2.132$ & $1.55 \mathrm{E}-15$ \\
\hline O15335 & 2 & Chondroadherin & $\Downarrow 2.112$ & $1.82 \mathrm{E}-14$ \\
\hline Q16610 & 5 & Extracellular matrix protein 1 & $\Downarrow 2.091$ & $6.00 \mathrm{E}-10$ \\
\hline Q08188 & 26 & Protein-glutamine gamma-glutamyltransferase E & $\Downarrow 2.090$ & $2.09 \mathrm{E}-04$ \\
\hline P07476 & 18 & Involucrin & $\Downarrow 2.057$ & $2.55 \mathrm{E}-06$ \\
\hline P62805 & 10 & Histone H4 & $\Downarrow 2.050$ & $2.96 \mathrm{E}-08$ \\
\hline P55072 & 10 & Transitional endoplasmic reticulum ATPase & $\Downarrow 2.035$ & $3.25 \mathrm{E}-11$ \\
\hline $\mathrm{P} 25398$ & 4 & 40S ribosomal protein $\mathrm{S} 12$ & $\Downarrow 1.975$ & $4.44 \mathrm{E}-16$ \\
\hline P13645 & 24 & Keratin, type I cytoskeletal 10 & $\Downarrow 1.974$ & $3.20 \mathrm{E}-04$ \\
\hline P48643 & 3 & T-complex protein 1 subunit epsilon & $\Downarrow 1.913$ & $6.05 \mathrm{E}-07$ \\
\hline P49189 & 3 & 4-trimethylaminobutyraldehyde dehydrogenase & $\Downarrow 1.897$ & $3.71 \mathrm{E}-04$ \\
\hline Q15149 & 4 & Plectin & $\Downarrow 1.896$ & $1.78 \mathrm{E}-15$ \\
\hline Q00610 & 7 & Clathrin heavy chain 1 & $\Downarrow 1.892$ & $3.09 \mathrm{E}-05$ \\
\hline P07951 & 3 & Tropomyosin beta chain & $\Downarrow 1.886$ & $6.40 \mathrm{E}-06$ \\
\hline P63104 & 10 & Protein kinase $\mathrm{C}$ inhibitor protein 1 & $\Downarrow 1.882$ & $8.76 \mathrm{E}-10$ \\
\hline P07900 & 5 & Heat shock protein HSP 90-alpha & $\Downarrow 1.871$ & $4.96 \mathrm{E}-06$ \\
\hline Q15366 & 2 & Poly (rC)-binding protein 2 & $\Downarrow 1.862$ & $2.79 \mathrm{E}-09$ \\
\hline P40394 & 4 & Alcohol dehydrogenase class $4 \mathrm{mu} /$ sigma chain & $\Downarrow 1.809$ & $3.25 \mathrm{E}-04$ \\
\hline P30838 & 7 & Aldehyde dehydrogenase, dimeric NADP-preferring & $\Downarrow 1.808$ & 7.64E-07 \\
\hline P13489 & 5 & Ribonuclease inhibitor & $\Downarrow 1.803$ & $9.43 \mathrm{E}-08$ \\
\hline $\mathrm{P} 07384$ & 2 & Calpain-1 catalytic subunit & $\Downarrow 1.791$ & $4.00 \mathrm{E}-15$ \\
\hline $\mathrm{P} 46940$ & 19 & Ras GTPase-activating-like protein IQGAP1 & $\Downarrow 1.791$ & $5.10 \mathrm{E}-06$ \\
\hline Q7Z406 & 4 & Myosin-14 & $\Downarrow 1.751$ & $7.46 \mathrm{E}-14$ \\
\hline A8K2U0 & 11 & Alpha-2-macroglobulin-like protein 1 & $\Downarrow 1.749$ & $1.23 \mathrm{E}-10$ \\
\hline P13797 & 6 & Plastin-3 & $\Downarrow 1.689$ & $2.11 \mathrm{E}-05$ \\
\hline P35579 & 52 & Myosin-9 & $\Downarrow 1.689$ & $7.61 \mathrm{E}-06$ \\
\hline B4DTG2 & 5 & Elongation factor 1-gamma & $\Downarrow 1.676$ & $5.69 \mathrm{E}-07$ \\
\hline $\mathrm{P} 17858$ & 5 & 6-phosphofructokinase, liver type & $\Downarrow 1.667$ & $5.46 \mathrm{E}-06$ \\
\hline P06576 & 11 & ATP synthase subunit beta, mitochondrial & $\Downarrow 1.667$ & $2.78 \mathrm{E}-04$ \\
\hline Q15084 & 5 & Protein disulfide-isomerase A6 & $\Downarrow 1.654$ & $2.76 \mathrm{E}-06$ \\
\hline P36952 & 6 & Serpin B5 & $\Downarrow 1.651$ & $5.93 \mathrm{E}-07$ \\
\hline $\mathrm{P} 07384$ & 7 & Calpain-1 catalytic subunit & $\Downarrow 1.650$ & $1.33 \mathrm{E}-05$ \\
\hline Q16851 & 6 & UTP--glucose-1-phosphate uridylyltransferase & $\Downarrow 1.634$ & $2.35 \mathrm{E}-07$ \\
\hline $\mathrm{P} 14625$ & 3 & Endoplasmin & $\Downarrow 1.615$ & $6.29 \mathrm{E}-04$ \\
\hline P09525 & 4 & Annexin A4 & $\Downarrow 1.606$ & $2.22 \mathrm{E}-14$ \\
\hline P10809 & 3 & $60 \mathrm{kDa}$ heat shock protein, mitochondrial & $\Downarrow 1.594$ & $2.07 \mathrm{E}-11$ \\
\hline $\mathrm{P} 60660$ & 8 & Myosin light polypeptide 6 & $\Downarrow 1.585$ & $2.84 \mathrm{E}-09$ \\
\hline Q9UHA7 & 7 & Interleukin-36 alpha & $\Downarrow 1.569$ & $2.18 \mathrm{E}-04$ \\
\hline Q9Y2V2 & 2 & Calcium-regulated heat stable protein 1 & $\Downarrow 1.564$ & $6.00 \mathrm{E}-15$ \\
\hline P13693 & 2 & Translationally-controlled tumor protein & $\Downarrow 1.526$ & $1.66 \mathrm{E}-07$ \\
\hline Q7L7L0 & 4 & Histone H2A type 3 & $\Downarrow 1.519$ & $4.72 \mathrm{E}-04$ \\
\hline Q06830 & 8 & Peroxiredoxin-1 & $\Downarrow 1.519$ & $5.99 \mathrm{E}-04$ \\
\hline P62879 & 2 & $\begin{array}{l}\text { Guanine nucleotide-binding protein } \mathrm{G}(\mathrm{I}) / \mathrm{G}(\mathrm{S}) / \mathrm{G}(\mathrm{T}) \\
\text { subunit beta-2 }\end{array}$ & $\Downarrow 1.517$ & $2.98 \mathrm{E}-10$ \\
\hline P30041 & 10 & Peroxiredoxin-6 & $\Downarrow 1.503$ & $1.13 \mathrm{E}-07$ \\
\hline
\end{tabular}

\section{$\dagger \Uparrow$ Represents upregulation and $\Downarrow$ Represents downregulation}




\section{CHAPTER 5}

Serum Bone Turnover and Angiogenesis Markers in Bisphosphonate-Related Osteonecrosis of the Jaw 


\section{Abstract}

Purpose: To evaluate serum markers of bone turnover, angiogenesis, endocrine, and inflammation in "Bisphosphonate-Related Osteonecrosis of the Jaw (BRONJ)" patients that could help provide insight into the pathogenesis of BRONJ development. Patients and Methods: Serum samples were obtained from 25 BRONJ patients and 48 controls without BRONJ. Samples were analyzed for total alkaline phosphatase (ALP); bone-specific alkaline phosphatase (BALP); osteocalcin (OCN); Ctelopeptide (CTX); vascular-endothelial growth factor (VEGF); triiodothyronine (T3); thyroxine (T4); thyroid-stimulating hormone (TSH); 25-hydroxyvitamin D; and C-reactive protein (CRP). At the time of study enrolment, BRONJ subjects had discontinued BP therapy for a mean period of 11.4 months, while controls continued to receive BP therapy.

Results: There was no significant difference in mean values of ALP $(98 \pm 52$ vs $85 \pm 76$ $\mathrm{U} / \mathrm{L}, \mathrm{p}=0.45)$; BALP (13 \pm 7 vs $14 \pm 8 \mu \mathrm{g} / \mathrm{L}, \mathrm{p}=0.58)$; osteocalcin $(12 \pm 4$ vs $14 \pm 10$ $\mathrm{ng} / \mathrm{mL}, \mathrm{p}=0.25)$; CTX (228 \pm 121 vs $222 \pm 119 \mathrm{pg} / \mathrm{mL}, \mathrm{p}=0.83) ; \mathrm{T} 3(118 \pm 29$ vs $110 \pm 30$ $\mathrm{ng} / \mathrm{dL}, \mathrm{p}=0.28) ; \mathrm{T} 4(10 \pm 2$ vs $9 \pm 2 \mu \mathrm{g} / \mathrm{dL}, \mathrm{p}=0.70) ; \mathrm{TSH}(2 \pm 1$ vs $2 \pm 2 \mathrm{mU} / \mathrm{L}, \mathrm{p}=0.21)$; and 25-hydroxyvitamin D ( $38 \pm 6$ vs $31 \pm 10 \mu \mathrm{g} / \mathrm{L}, \mathrm{p}=0.16$ ); and CRP (20 \pm 19 vs $13 \pm 8$ $\mathrm{mg} / \mathrm{L}, \mathrm{p}=0.20$ ) between BRONJ cases and controls. However, VEGF levels were significantly decreased in controls compared to BRONJ cases (256 \pm 174 vs $422 \pm 436$ $\mathrm{pg} / \mathrm{mL}, \mathrm{p}=0.02)$.

Conclusions: Angiogenesis makers are significantly reduced in patients receiving BP therapy and these effects may be reversed following discontinuation of BP's. The role 
of impaired angiogenesis in BRONJ development needs to be explored in future prospective studies. 


\section{Introduction}

Bisphosphonate's (BP), potent inhibitor of osteoclast mediated bone resorption, are associated with bisphosphonate-related osteonecrosis of the jaw (BRONJ).[103,120] To date, the etiology and pathogenesis of BRONJ development remains unknown. It is hypothesized that long-term BP therapy may impair bone turnover and angiogenesis process leading to BRONJ development.

Bone turnover or bone remodeling is an essential process required for maintaining skeletal health. It begins with the resorption of old bone by osteoclasts, followed by the formation of new bone by osteoblasts.[186] During bone remodeling, osteoblasts respond to structural damage or hormonal influence by expressing osteoclastogenesis cytokines[187,188] that promote osteoclast formation and subsequent resorption. Consequently, osteoclastic bone resorption leads to the release of osteotropic factors[147,189] that stimulate bone formation. This intercellular communication between osteoclasts and osteoblasts is crucial for bone renewal. Hence, the process of bone remodeling is often referred to as being "coupled", meaning bone formation is linked to bone resorption. It is suggested that over suppression of osteoclasts by long-term BP therapy can affect osteoblast function impairing bone renewal.[20] Recently, several cases of osteonecrosis of the jaw have also been reported in patients treated with denosumab, a RANK-ligand inhibitor that results in osteoclast suppression.[190] In the absence of effective bone resorption, conditions like tooth extraction and dental infection might result in tissue death, vascular loss, and eventually osteonecrosis. 
BP's alternative pharmacological actions include angiogenesis inhibition[58,59] and immune modulation.[191] Treatment with pamidronate or zoledronate has been reported to reduce vascular endothelial growth factor (VEGF) levels[58,59] and bone blood flow in rats.[61] In vitro studies have documented BP's to reduce endothelial cell proliferation and decrease capillary-like tube formation.[33] Collectively, these findings suggest that BP's anti-angiogenic activity in jawbones could induce avascular necrosis and impair tissue repair.

Assessing bone turnover and angiogenesis markers appears to be valuable approaches for the early diagnosis and management of BRONJ. During bone remodeling, biochemical markers of bone turnover are released into circulation.[186] They comprise of enzymes/proteins secreted by osteoclasts and osteoblasts, degradation products formed during bone resorption, and precursors released during new bone formation. Similarly, during angiogenic process, various proteins including growth factors are released into circulation.[192] This study aims at serologically characterizing bone turnover, angiogenesis, endocrine, and inflammatory markers in BRONJ patients that could help provide insight into the pathogenesis of BRONJ development. 


\section{Patients and Methods}

\section{Subjects}

The University of Minnesota Institutional Review Board approved this case-control study and written informed consent was obtained from all subjects. Cancer patients above 30 years of age who had received intravenous BP therapy for cancer management were included in this study. Patients with a history of either radiation therapy to the head and neck region or neoplasm's (including metastasis) involving the head and neck region were excluded. Eligible cases were defined as patients with a confirmed diagnosis of BRONJ. The American Association of Oral and Maxillofacial Surgeons criteria[85] was used to diagnose BRONJ: “exposed bone in the maxillofacial area occurring in the absence of head and neck irradiation and showing no evidence of healing for at least 8 weeks after identification in patients treated with BP therapy". The control group consisted of cancer patients who had received at least 10 intravenous $\mathrm{BP}$ infusions without a history of BRONJ.

At enrollment, all study subjects underwent a comprehensive dental examination including oral cancer screening. Serum samples were collected and stored at $-80^{\circ} \mathrm{C}$. 


\section{Laboratory Procedures}

Serum samples were assayed for total alkaline phosphatase (ALP) ${ }^{\dagger}$; bone-specific alkaline phosphatase (BALP) $)^{\ddagger}$; osteocalcin $(\mathrm{OCN})^{\dagger}$; C-telopeptide (CTX) endothelial growth factor $(\mathrm{VEGF})^{\S}$; triiodothyronine $(\mathrm{T} 3)^{\dagger}$; thyroxine $(\mathrm{T} 4)^{\dagger}$; thyroidstimulating hormone $(\mathrm{TSH})^{\dagger} ; 2$-hydroxyvitamin $\mathrm{D}^{\dagger}$; and C-reactive protein $(\mathrm{CRP})^{\dagger}$.

${ }^{\dagger}$ Fairview Health Services, Minneapolis, MN

${ }^{*}$ Immunodiagnostic Systems Ltd

${ }^{\S}$ Quantikine ELISA Kit, R\&D Systems, Minneapolis, MN

\section{Statistical Analysis}

Two-sided student's $t$ test was used for comparing differences between BRONJ cases and controls.

\section{Results}

\section{Patient Characteristics}

Table 1 lists select subject characteristics. There were no significant differences in age $(\mathrm{P}=0.50)$, sex $(\mathrm{P}=0.60)$, or race $(\mathrm{P}=1.00)$ between $\mathrm{BRONJ}$ cases and controls. The mean number of BP infusions was significantly higher in BRONJ cases compared to controls (38.4 \pm 26.3 infusions versus 18.8 $\pm 7.2 ; \mathrm{P}<0.0001)$. BRONJ cases also received BP therapy for a longer period (mean, $45.1 \pm 29.7$ months versus $37.7 \pm 21.9$ 
months), although the difference was insignificant $(\mathrm{P}=0.23)$. Most patients had received BP therapy for metastatic breast cancer management (13 BRONJ and 27 controls, $\mathrm{p}=0.02$ ).

Notably, at the time of sampling, BRONJ subjects had discontinued BP therapy for a mean period of 11.4 months (range, 2 to 30 months) following initial BRONJ diagnosis, while controls continued to receive BP therapy for cancer management.

\section{Laboratory Results}

Mean serum levels of bone turnover, angiogenesis, endocrine, and inflammatory markers are shown in Table 2. There was no significant difference in mean values of ALP ( $98 \pm 52$ vs $85 \pm 76 \mathrm{U} / \mathrm{L}, \mathrm{p}=0.45)$; BALP ( $13 \pm 7$ vs $14 \pm 8 \mu \mathrm{g} / \mathrm{L}, \mathrm{p}=0.58)$; osteocalcin (12 \pm 4 vs $14 \pm 10 \mathrm{ng} / \mathrm{mL}, \mathrm{p}=0.25)$; CTX $(228 \pm 121$ vs $222 \pm 119 \mathrm{pg} / \mathrm{mL}, \mathrm{p}=0.83) ; \mathrm{T} 3$

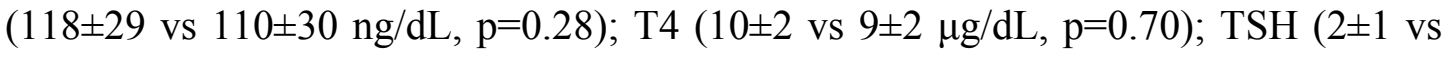
$2 \pm 2 \mathrm{mU} / \mathrm{L}, \mathrm{p}=0.21$ ); and 25-hydroxyvitamin $\mathrm{D}(38 \pm 6$ vs $31 \pm 10 \mu \mathrm{g} / \mathrm{L}, \mathrm{p}=0.16)$; and CRP $(20 \pm 19$ vs $13 \pm 8 \mathrm{mg} / \mathrm{L}, \mathrm{p}=0.20)$ between BRONJ cases and controls. However, VEGF levels were significantly decreased in controls compared to BRONJ cases ( $422 \pm 436$ vs $256 \pm 174 \mathrm{pg} / \mathrm{mL}, \mathrm{p}=0.02)$.

\section{Discussion}

Despite several documented case reports, retrospective clinical studies, and efforts to develop animal models, the pathogenesis of BRONJ development remains 
unknown. Disagreement exists in the literature regarding the role of suppressed bone turn over and angiogenesis in BRONJ development. The present study evaluated serum bone turnover, angiogenesis, endocrine, and inflammatory markers and found no significant differences in bone turnover, endocrine, and inflammatory markers between controls and BRONJ cases. However, the mean VEGF values were significantly decreased in controls compared to BRONJ cases.

\section{Bone turnover markers}

Biochemical markers of bone turnover provide insight into the dynamic changes of the skeleton.[186] They are valuable tools in monitoring the effect of antiresorptive therapy[193], predicting bone loss and fracture in osteoporosis[186], identifying the progression of joint damage in rheumatoid arthritis[194], and in ascertaining the extent of bone involvement in metastatic cancer and multiple myeloma.[195,196]

Bone resorption markers: Among the bone resorption markers, CTX is a highly sensitive indicator of bone resorption.[193] It is a degradation product of $\alpha 1$ chain C telopeptide of type I collagen that is released into bloodstream during bone resorption and is easily measured by ELISA.[197] Several reports have suggested a relationship between suppressed levels of serum CTX and the risk of developing BRONJ.[198,199,200] In one of the earliest reports, Marx et al. retrospectively evaluated $30 \mathrm{BRONJ}$ patients receiving oral $\mathrm{BP}$ and described that serum values of 
$<100 \mathrm{pg} / \mathrm{mL}$ represented a high risk for BRONJ development, values between 100 $\mathrm{pg} / \mathrm{mL}$ to $150 \mathrm{pg} / \mathrm{mL}$ represented moderate risk, and values $>150 \mathrm{pg} / \mathrm{mL}$ represented minimal risk.[198] In Marx's study, no control group was included and only 17 out of 30 patients completed the CTX test. Similarly, Kwon et al. retrospectively evaluated 18 BRONJ patients treated with oral BP and established a correlation between low CTX levels and the number of BRONJ lesions and BRONJ staging.[199] Lazarovici et al. reported that a CTX value of $<150 \mathrm{pg} / \mathrm{mL}$ was significantly associated with BRONJ development, with an increased odds ratio of $5.27(\mathrm{p}=0.004)$.[200] In contrast, Kunchur et al. evaluated patients on oral BP $(n=215)$ and intravenous BP $(n=7)$ scheduled for tooth extractions and reported that CTX test did not predict the development of BRONJ, however, a value of less than 150 to $200 \mathrm{pg} / \mathrm{mL}$ suggested patients in the "risk zone".[201] In two different studies, Bagan et al. evaluated patients receiving oral[202] and intravenous BP's[203] and found no relationship between serum CTX and the risk of developing BRONJ.

Previous studies demonstrate that following intravenous BP administration, bone resorption markers rapidly decrease reaching a maximum suppression within a few days.[71,204] However, there is no evidence that continued treatment with intravenous BP's results in more extensive or progressive suppression of bone resorption markers leading to BRONJ development. In the Health Outcomes and Reduced Incidence with Zoledronic Acid Once Yearly (HORIZON) Pivotal Fracture Trial, CTX values decreased immediately after initial infusion and remained below the threshold with no progressive decline over 3-year study period.[71] In a 
randomized phase III trial assessing long-term efficacy and safety of zoledronate in bone metastases patients, bone resorption markers showed no evidence of progressive suppression over 2-year period with continued use of $4 \mathrm{mg}$ or $8 \mathrm{mg}$ of zoledronate every 3 weeks.[204] Similarly, in osteoporosis patients, no progressive decrease in bone turnover markers was noted over the span of 10 years of alendronate use.[205] It is suggested that bone resorption marker levels rapidly decrease following BP administration, however, their level plateau over time remaining below the threshold level.

Furthermore, little is known about the values of bone turnover makers following discontinuation of long-term BP therapy in BRONJ patients. Two longterm studies noted that following discontinuation of alendronate therapy, the mean values of bone remodeling markers remained below base-line levels after 10 years.[205,206] A recent study noted that the antiresorptive effects of zoledronate lasted for at least five years in HIV-infected men, inquiring the need for frequent administration of zoledronate.[207] Similarly, a randomized clinical trial in postmenopausal women noted that a single dose of $5 \mathrm{mg}$ zoledronate stably decreased bone turnover and increased bone mineral density, which lasted for at least 5 years.[208] Throughout the 5-year trial, mean level of resorption markers remained at least $40 \%$ lower in the zoledronate group compared to placebo group. In the present study, there was no significant difference in CTX values between BRONJ cases who discontinued BP therapy and controls who continued receiving BP therapy $(228 \pm 121$ vs $222 \pm 119 \mathrm{pg} / \mathrm{mL}, \mathrm{p}=0.83$ ). A plausible explanation for this insignificant difference 
is that, following discontinuation of BP therapy CTX levels may remain suppressed and never reach the baseline values in BRONJ subjects, which is consistent with the previous BP efficacy studies.[205,206]'[208] Once incorporated into bones, BP's have a prolonged skeletal half-life of which is estimated to be around 10 years.[26]

Bone formation markers: Previous studies have also evaluated bone formation markers in reference to BRONJ development.[200,209,210] In a prospective study investigating the predictive value of BALP, CTX, and parathyroid hormone in BRONJ development, Lazarovici et al. noted that mean BALP level was significantly lower in BRONJ patients treated with oral BP compared to non-BRONJ patients receiving oral $\mathrm{BP}(12.60$ versus $20.84 \mathrm{pg} / \mathrm{mL}, \mathrm{p}=<0.05)$.[200] No firm conclusions could be drawn from these findings as only 4 out of 51 patients treated with oral BP developed BRONJ. Among patients receiving intravenous BP therapy, the mean BALP level did not differ significantly between BRONJ and non-BRONJ patients (22.32 versus $20.78 \mathrm{pg} / \mathrm{mL}, \mathrm{p}=>0.05)$.

In a retrospective study, Kwon et al. noted that the mean serum osteocalcin levels were markedly reduced than the assay reference range $(1.91 \pm 1.51 \mathrm{ng} / \mathrm{mL}$ versus 3.2 to $12.2 \mathrm{ng} / \mathrm{mL}$ ) in $23 \mathrm{BRONJ}$ patients receiving oral BP therapy.[209] A lack of established control group in this study limited the comparison of serum osteocalcin levels between BRONJ and non-BRONJ patients. Lehrer et al., in their retrospective study of seven patients, reported that serum BALP and osteocalcin 
levels were within the normal reference range.[210] Furthermore, in an exploratory gene expression-profiling study, Raje et al. noted that genes involved in osteoclast and osteoblast signaling, activation, or differentiation were significantly downregulated in BRONJ patients compared to non-BRONJ patients $(\mathrm{P}<0.05)$.[211] Consistent with the gene expression results, serum bone formation and bone resorption markers were decreased in BRONJ patients, indicating impaired bone homeostasis in BRONJ subjects at both the transcriptional and protein level.

In the present study, we found no significant difference in mean ALP $(98 \pm 52$ vs $85 \pm 76 \mathrm{U} / \mathrm{L}, \mathrm{p}=0.45)$; BALP ( $13 \pm 7$ vs $14 \pm 8 \mu \mathrm{g} / \mathrm{L}, \mathrm{p}=0.58)$; osteocalcin $(12 \pm 4$ vs $12 \pm 7 \mathrm{ng} / \mathrm{mL}, \mathrm{p}=0.99)$ levels between BRONJ cases and controls. These results could be explained by the fact that the bone resorption and bone formation markers remain suppressed for several years after discontinuation of BP therapy,[206,207] invalidating the difference between patients who continue or discontinue BP therapy.

\section{Angiogenesis markers}

It has been hypothesized that angiogenesis suppression by BP's might play an important role in BRONJ development. Previous studies have shown BP's to inhibit angiogenesis in bone and prostate tissues of murine models.[212,213] Similarly, clinical studies have shown BP's to inhibit angiogenesis and tumor cell bone invasion in cancer patients by reducing circulating levels of VEGF, basic fibroblast growth factor (bFGF), metalloproteinase 2 (MMP-2).[58,214] VEGF are not only important for endothelial cell proliferation, angiogenesis, and capillary permeability,[215] they 
also stimulate bone repair by promoting bone turnover.[216] Inactivation of VEGF has shown to decrease blood vessel invasion into growth plates associated with impaired trabecular bone formation,[217] decreased callus mineralization in femoral fractures, and subdued healing of tibial cortical bone defects.[216] These results are consistent with the finding that VEGF also plays a direct autocrine role in osteoblast differentiation.[216]

Recently, a clinical study by Vincenzi et al. reported that anti-angiogenic properties of $\mathrm{BP}$ are directly linked to BRONJ pathogenesis. Six of the 81 patients who developed BRONJ in this study (after a median number of 9 infusions), showed a significant decrease in circulating VEGF levels at day 7 and day 21 after the first BP administration. However, it is unclear if BRONJ patients in this study had concurrently received other anti-angiogenic agents for cancer treatment. An immunohistochemical study by Wehrhan et al. reported that angiogenesis, but not vascularization, was impaired in mucoperiosteal tissues adjacent to BRONJ lesions. The authors indicate that tissue regeneration in BRONJ patients may be impaired due to subdued vessel remodeling and neovessel formation.[218] Furthermore, a possible haplotype effect of VEGF polymorphism has been reported in female BRONJ patients.[219]

A few recent studies have also reported cases of exposed jaw bone, very similar to BRONJ, in patients treated with sunitinib and bevacizumab.[220,221,222,223,224] Both sunitinib and bevacizumab are anti- 
angiogenic agents used in cancer treatment. Sunitinib is a tyrosine kinases inhibitor that targets receptors of VEGF, platelet-derived growth factor (PDGF), c-kit, FLT3 and RET.[225] Bevacizumab is a recombinant human monocloncal antibody that inhibits VEGF-A.[226] It is estimated that BRONJ frequency is higher in patients receiving combined $\mathrm{BP}$ and antiangiogenic treatment compared to patients receiving BP therapy alone.[220]

In the present study, VEGF levels were significantly decreased in controls receiving BP therapy compared to BRONJ patients who had discontinued BP therapy for a mean period 11.4 months ( $256 \pm 174$ vs $422 \pm 436 \mathrm{pg} / \mathrm{mL}, \mathrm{p}=0.02)$. BP's are known to reduce circulating VEGF levels in bone metastasis patients.[58,227] Although the precise mechanism is not completely understood, it is suggested that BP's may elicit several antiangiogenic-related cytokine patterns and cascades that can be probably mediated by the inhibition of isoprenylation of intracellular molecular targets (likely small GTP-binding proteins). Recent evidence indicate that zoledronate inhibit endothelial cell adhesion, migration and survival through the suppression of multiple, prenylation-dependent signaling pathways.[228,229] Kinases such as ERK1/2, JNK, Rock, FAK and PKB are affected by zoledronate in a prenylationdependent manner.[228] Collectively, these findings may explain the decreased serum VEGF levels noted in controls in the present study. However, the effect of discontinuation of BP therapy on VEGF levels is unknown. A previous study noted reversal of diminished VEGF-related glomerulonephritis following discontinuation of bevacizumab and zoledronate.[230] Since BP's have direct action on VEGF, 
discontinuation of BP therapy may increase VEGF levels, which explains the elevated VEGF levels seen in BRONJ cases compared to controls in the present study.

There was no wide difference in chemotherapeutic regimen between BRONJ cases and controls including antiangiogenic agents in the present study. However, it is unknown if chemotherapy has confounding effects on angiogenesis markers.

\section{Other Markers}

Thyroid hormone is essential for skeletal development and plays an important role in maintaining optimal bone strength. Our previous retrospective chart review study indicated that hypothyroidism was an important risk factor for BRONJ development $(\mathrm{HR}=3.59 ; 95 \% \mathrm{CI}=1.31-9.83 ; \mathrm{p}=0.013)$. However, in the present study there were no significant differences in $\mathrm{T} 3, \mathrm{~T} 4$, and TSH levels between BRONJ cases and controls. The differences in results between the two studies could be attributed to the retrospective nature of the chart review study. Furthermore, there was no significant difference in the total 25 -hydroxyvitamin D and CRP levels between BRONJ cases and controls.

\section{Conclusion}

The risk of developing BRONJ increases with cumulative doses of BP and the type of BP administered. A higher cumulative dose of BP's could conceivably induce 
a profound adverse effect on bone turnover and angiogenesis markers. To date, no prospective clinical studies have evaluated the role of bone turn over markers in BRONJ development. Most reports that have suggested an association between impaired levels of bone turnover and BRONJ development are retrospective studies and cross-sectional studies, which are subjected to bias. In milieu of the conflicting results, several investigators including the American Society for Bone and Mineral Research Task Force on BRONJ have refuted CTX test as a predictor for BRONJ development.[231,232] Clinicians need to be aware of the lack of high-level evidence when utilizing CTX test for assessing risk of BRONJ development or for predicting BRONJ progression.

Angiogenesis suppression by BP's might play an important role in BRONJ development. BP's anti-osteoclastic activities could indirectly diminish skeletal vasculature leading to avascular necrosis and impaired tissue repair. The role of impaired angiogenesis in BRONJ development needs to be further explored in future prospective trials. 
Table 1. Baseline patient characteristics $(\mathrm{N}=73)$

\begin{tabular}{|c|c|c|c|}
\hline Characteristic & $\begin{array}{c}\text { BRONJ } \\
(\mathrm{N}=25)\end{array}$ & $\begin{array}{l}\text { Non-BRONJ } \\
(\mathrm{N}=48)\end{array}$ & $P \dagger$ \\
\hline Age - mean \pm sd, in years & $66.3 \pm 10.3$ & $64.4 \pm 11.7$ & 0.50 \\
\hline $\begin{array}{l}\text { Sex }- \text { no. }(\%) \\
\text { Female }\end{array}$ & $16(64 \%)$ & $34(71 \%)$ & 0.60 \\
\hline $\begin{array}{l}\text { Race }- \text { no. }(\%) \\
\text { White } \\
\text { Black }\end{array}$ & $\begin{array}{c}24(96 \%) \\
1(4 \%)\end{array}$ & $\begin{array}{c}46(96 \%) \\
2(4 \%)\end{array}$ & 1.00 \\
\hline $\begin{array}{l}\text { Malignant Disease - no. (\%) } \\
\text { Breast cancer } \\
\text { Multiple myeloma } \\
\text { Prostate cancer } \\
\text { Lung cancer } \\
\text { Renal cell carcinoma } \\
\text { Others }\end{array}$ & $\begin{array}{l}13(52 \%) \\
4(16 \%) \\
2(8 \%) \\
2(8 \%) \\
3(12 \%) \\
1(4 \%)\end{array}$ & $\begin{array}{l}27(56 \%) \\
15(31 \%) \\
6(13 \%) \\
0(0 \%) \\
0(0 \%) \\
0(0 \%)\end{array}$ & 0.02 \\
\hline $\begin{array}{l}\text { Pre-existing medical conditions - no. ( } \\
\text { Diabetes mellitus } \\
\text { Hypertension } \\
\text { Hypercholesterolemia/Hyperlipidemia } \\
\text { Hypothyroidism }\end{array}$ & $\begin{array}{c}1(4 \%) \\
11(44 \%) \\
11(44 \%) \\
7(28 \%)\end{array}$ & $\begin{array}{l}5(10 \%) \\
26(54 \%) \\
15(33 \%) \\
10(21 \%)\end{array}$ & $\begin{array}{l}0.66 \\
0.47 \\
0.45 \\
0.56\end{array}$ \\
\hline $\begin{array}{l}\text { Type of Bisphosphonates }{ }^{\star}-\text { no. }(\%) \\
\text { Zoledronate } \\
\text { Pamidronate } \\
\text { Pamidronate }+ \text { Zoledronate }\end{array}$ & $\begin{array}{l}16(64 \%) \\
2(8 \%) \\
7(28 \%)\end{array}$ & $\begin{array}{c}32(67 \%) \\
6(13 \%) \\
10(21 \%)\end{array}$ & 0.71 \\
\hline $\begin{array}{l}\text { Bisphosphonate infusions }- \text { mean } \pm \text { sd } \\
\text { All cancer }\end{array}$ & $38.4 \pm 26.3$ & $18.8 \pm 7.2$ & $<0.0001 *$ \\
\hline \multicolumn{4}{|l|}{$\begin{array}{l}\text { Duration of Bisphosphonate exposure - } \\
\text { mean } \pm \text { sd, in months }\end{array}$} \\
\hline All cancer & $45.1 \pm 29.7$ & $37.7 \pm 21.9$ & 0.23 \\
\hline
\end{tabular}

$\dagger$ P-values were calculated from a Fisher's exact test or Pearson's chi-squared test for categorical variables and two-sample t-tests for continuous variables.

$* \mathrm{P}=0.0011$ allowing unequal variances in the two groups 
Table 2: Serum Biochemical Markers

\begin{tabular}{|c|c|c|c|c|}
\hline Biochemical markers & $\begin{array}{l}\text { BRONJ } \\
(\mathbf{N}=25)^{\dagger}\end{array}$ & $\begin{array}{c}\text { Non-BRONJ } \\
(\mathrm{N}=48)\end{array}$ & Reference range & $P \dagger$ \\
\hline \multicolumn{5}{|l|}{ Bone formation } \\
\hline Total alkaline phosphatase & $98 \pm 52$ & $85 \pm 76$ & 40 to $150 \mathrm{U} / \mathrm{L}$ & 0.45 \\
\hline Bone-specific alkaline phosphatase & $13 \pm 7$ & $14 \pm 8$ & 6.5 to $22 \mu \mathrm{g} / \mathrm{L}$ & 0.58 \\
\hline Osteocalcin & $12 \pm 4$ & $14 \pm 10$ & 11 to $50 \mathrm{ng} / \mathrm{mL}$ & 0.25 \\
\hline \multicolumn{5}{|l|}{ Bone resorption } \\
\hline C-telopeptide & $228 \pm 121$ & $222 \pm 119$ & 50 to $580 \mathrm{pg} / \mathrm{mL}$ & 0.83 \\
\hline \multicolumn{5}{|l|}{ Angiogenesis } \\
\hline Vascular-endothelial growth factor & $422 \pm 436$ & $256 \pm 174$ & 60 to $707 \mathrm{pg} / \mathrm{mL}$ & 0.02 \\
\hline \multicolumn{5}{|l|}{ Endocrine } \\
\hline Triiodothyronine & $118 \pm 29$ & $110 \pm 30$ & 60 to $180 \mathrm{ng} / \mathrm{dL}$ & 0.28 \\
\hline Thyroxine & $10 \pm 2$ & $9 \pm 2$ & 5 to $11 \mu \mathrm{g} / \mathrm{dL}$ & 0.70 \\
\hline Thyroid-stimulating hormone & $2 \pm 1$ & $2 \pm 2$ & 0.4 to $3.0 \mu \mathrm{IU} / \mathrm{mL}$ & 0.21 \\
\hline 25-hydroxyvitamin D & $38 \pm 6$ & $31 \pm 10$ & 30 to $75 \mu \mathrm{g} / \mathrm{L}$ & 0.16 \\
\hline \multicolumn{5}{|l|}{ Inflammation } \\
\hline C-reactive protein & $20 \pm 19$ & $13 \pm 8$ & 0 to $10 \mathrm{mg} / \mathrm{L}$ & 0.20 \\
\hline
\end{tabular}

$\uparrow$ Missing data for 1 BRONJ subject 


\section{CHAPTER 6}

Biomechanical Properties of Bisphosphonate-Treated Rat Mandible 


\begin{abstract}
Objectives: Previous studies have evaluated the effects of long-term bisphosphonate (BP) therapy on the biomechanical properties of long bones, however, BP's effects on the biomechanical properties of jaw bones remains unknown. This study investigates changes in biomechanical properties within rat mandibles compared to femurs following long-term BP treatment.
\end{abstract}

Methods: For 12 weeks, 2.5-month-old male Sprague-Dawley rats $(n=13)$ were injected with saline (control), zoledronate $0.06 \mathrm{mg} / \mathrm{kg}$ or zoledronate $0.12 \mathrm{mg} / \mathrm{kg}$. Multiple measures of Vickers microhardness and nanohardness were performed on each harvested trabecular and cortical bone of mandibles and femurs. Crack analysis from Vickers indentation testing was used to determine fracture toughness of dehydrated cortical bones. Structural features of the tested bones were evaluated by scanning electron microscopy (SEM) and histology. General Linear Model (GLM) univariate analysis (bone and measurement as fixed and random factors, respectively) with Tuckey post-hoc tests determined statistically significant differences $(p<0.05)$.

Results: In all cases the zoledronate-treated bones were significantly harder than the saline-treated bones, but treatment with zoledronate reduced bone fracture toughness. SEM revealed higher osteon density in zoledronate-treated bones than in salinetreated bones, which may account for the aforementioned biomechanical differences. There was no observed difference in bone properties between the two zoledronatetreated groups. The anterior region of the mandible was significantly harder compared to the posterior region within the zoledronate groups. 
Conclusions: Long-term BP treatment results in increased hardness and brittleness within both mandible and femur rat bones. These changes in biomechanical properties can lead to increased microdamage accumulation within the jaw bones leading to BRONJ development. 


\section{Introduction}

Bisphosphonate's (BP), potent antiresorptive agents, are the standard of care for patients with osteoporosis, Paget's disease, bone metastasis and multiple myeloma.[9,12,22,23,127] They reduce bone turnover rate by inhibiting osteoclast mediated bone resorption.[11,205] Recently, long-term BP therapy has been associated with the dental complication bisphosphonate-related osteonecrosis of the jaw (BRONJ).[103,120] To date, the etiology and pathogenesis of BRONJ development remains unknown. It is hypothesized that long-term BP therapy may severely suppress bone turn over rate leading to impaired biomechanical properties of bone resulting in BRONJ development.

In humans, skeleton is continually renewed by a process called bone turnover or bone remodeling, which involves osteoclast-mediated bone resorption, osteoblast-mediated bone formation, and re-mineralization of newly formed bone. By reducing bone turnover rate, BP's prolong the lifespan of bone structural units (BSUs) and the secondary mineralization phase, which in turn increases bone mineral density.[233] Some studies attribute decrease in osteoporotic fracture incidence to increased degree of mineralization rather than increased bone mass activated by BP therapy.[233,234] However, overly suppressing bone turnover rate can impair biomechanical properties of bone. Chronic over suppression of bone turnover results in hypermineralized bones that are very brittle and susceptible to fracture.[235,236] Also, over suppression of bone turnover may inhibit repair of microscopic cracks $(30-80 \mathrm{~mm})$ which usually occurs in bone as result of physiologic stress. ${ }^{12,13,[237] ~ M i c r o d a m a g e d ~ b o n e s ~ a r e ~}$ 
susceptible to necrosis when there is increased demand for osseous repair owing to trauma or infection.[20] Thus, it is critical to understand the effects of long-term BP therapy on bone turnover and its implication in BRONJ development.

Previous studies have evaluated the effects of long-term BP therapy on the biomechanical properties of long bones, however, BP's effects on the biomechanical properties of jaw bones remains unknown. This study investigates changes in biomechanical properties within rat mandibles compared to femurs following longterm BP treatment.

\section{Materials and Methods}

\section{Animals and Drug Administration}

The University of Minnesota Institutional Animal Care and Use Committee approved the experimental protocol. For 12 weeks, 2.5-month-old male Sprague-Dawley rats $(\mathrm{n}=13)$ received intraperitoneal injections of saline (control), zoledronate $0.06 \mathrm{mg} / \mathrm{kg}$ or zoledronate $0.12 \mathrm{mg} / \mathrm{kg}$. Commercially available zoledronate (Zometa ${ }^{\circledR}$; Novartis Pharmaceuticals Corporation, East Hanover, NJ) in the form of 4-mg vials was diluted with sterile saline solution and injected intraperitoneal. The dose of zoledronate treatment for rats was extrapolated from the amount of drug prescribed to cancer patients with skeletal metastasis. Assuming that a cancer patient of $\sim 70 \mathrm{Kg}$ receives a recommended dose of $4 \mathrm{mg}$ of drug every 4 weeks, which corresponding to a dose of $0.06 \mathrm{mg} / \mathrm{Kg}$, we studied the effects of zoledronate at two different doses of 
$0.06 \mathrm{mg} / \mathrm{kg}$ and $0.12 \mathrm{mg} / \mathrm{kg}$. All animals were given a regular diet throughout the duration of the experiment. After 12 weeks of treatment, the animals were sacrificed, and mandibles and femurs were harvested.

\section{Specimen Preparation}

The harvested mandibles were separated at the symphysis and incisor teeth removed. Femoral neck and shaft cross-sections were also obtained. The mandible and femur specimens were embedded in Polymethyl methacrylate (PMMA) and polished using polishing paper (SiC, P1200) with micropolish alumina suspension (0.1 and $0.05 \mu \mathrm{m}$, respectively). For femur, trabecular measurements were obtained from the neck area, while cortical measurements were obtained from the shaft region. For Mandible, trabecular measurements were obtained from the area around the roots, while cortical measurements were obtained from the anterior and posterior regions. Anterior mandible was defined as area above the mental foramen and posterior mandible was defined as area under the third and first molars, and above the mandibular canal.

\section{Biomechanical testing}

Nanoindentation: Nanoindentation measurements were performed on a nanomechanical indenter system (Nano Indenter XP, MTS Systems Corporation) with a diamond Berkovich indentation tip. The system was calibrated using a fused quartz standard and its nanoindentation modulus and hardness was calculated as $74.0 \pm 3.2$ 
and 10.0 $\pm 0.5 \mathrm{GPa}$, respectively. The embedded mandible and femur bone samples were glued on to aluminum stubs directly and 30 indents were performed on each trabecular and cortical bone with an indent spacing of $5 \mu \mathrm{m}$. During nanohardness testing, the specimens were kept hydrated and a maximum indentation depth of 2000 $\mathrm{nm}$ was used. The elastic modulus and hardness were obtained from the curves using the Oliver-Pharr method.

Microindentation: The Vickers hardness of specimens was measured with a microhardness testing machine (Micromet 5104, Buehler, USA). The indenter load was $100 \mathrm{~g}$ and the load-holding time was 10 seconds. Ten random points on each sample were tested and averaged.

For Crack analysis test, dehydrated bone samples were utilized. For the dehydrated specimens, the maximum load was $1 \mathrm{Kg}$ with a 10 second hold time at maximum loading.

\section{Scanning Electron Microscopy (SEM) Analysis}

The air-dried samples were mounted on aluminum stubs with double-faced carbon tape. The surface morphologies of samples were analyzed using Hitachi desktop scanning electron microscope (SEM TM-3000, Hitachi, Japan) at $15 \mathrm{kV}$ of the accelerating voltage. 


\section{Histology}

The mandible and femur samples were fixed in $10 \%$ buffered formalin solution and later decalcified using 10\% EDTA. The specimens were processed for paraffin embedding, and sections were cut and stained with hematoxylin and eosin (H\&E). Histology slides were used to evaluate structural features of the BP treated and saline bone samples.

\section{Statistical Analysis}

General Linear Model (GLM) univariate analysis (bone and measurement as fixed and random factors, respectively) with Tuckey post-hoc tests determined statistically significant differences $(\mathrm{p}<0.05)$.

\section{Results}

All animals completed the treatment without any observable complications from the drug.

Microindentation results: Following 12-weeks of treatment, zoledronate significantly increased the hardness of both trabecular and cortical bones in the mandible and femur compared to control group (Figure 1). In addition, zoledronate treatment reduced bone fracture toughness in both the mandible and femur. There was no 
significant difference in any of the bone properties between $0.06 \mathrm{mg} / \mathrm{kg}$ and $0.12 \mathrm{mg} / \mathrm{kg}$ zoledronate groups. Furthermore, in the zoledronate treated animals, we noted that the mandible was harder than the femur, although the difference was statistically insignificant $(\mathrm{P}=0.18$, Figure 2$)$. Also, the anterior region of the mandible was significantly harder compared to the posterior region ( $\mathrm{P}=0.007$, Figure 3 ).

\section{Nanoindentation results:}

Similar to microindentation, nanoindentation revealed that zoledronate treatment significantly increased the hardness of femur trabecular bone compared to control group. There was no difference in the bone properties between the two-zoledronate groups. Furthermore, the modulus of elasticity was significantly higher in the zoledronate-treated groups, corresponding to harder bone.

\section{Scanning Electron Microscopy results:}

SEM revealed higher osteon density in zoledronate-treated bones compared to salinetreated bones (Figure 4), which may account for the aforementioned biomechanical differences.

\section{Histology}

Histological image shows dense bone within the zoledronate group compared to the saline group (Figure 5). 


\section{Discussion}

In the present study, long-term zoledronate treatment resulted in altered biomechanical properties. Long-term zoledronate treatment not only increased trabecular and cortical bone hardness, it also increased bone brittleness. These differences were more significant in the mandible compared to femur and were not dose dependent. The higher osteon density noted in zoledronate-treated bones may account for the above-mentioned biomechanical differences.

Similar to our report, previous animal studies have noted altered bone mechanical properties with BP treatment.[238,239,240] Pozzi et al. noted that high dose zoledronate treatment increased bone mineral density, bone mineral content, trabecular bone volume and cortical bone thickness.[238] These effects were not dose dependent, suggesting that lower doses of zoledronate is adequate enough to inhibit bone resorption. In addition, mechanical testing revealed that increased bone stiffness was associated with increased bone brittleness, which is similar to our findings. Furthermore, it was noted that high doses of zoledronate interfere with both osteoblast and osteoclast lineages, resulting in possible inhibition of both cell types.

It is well recognized that mineral content and matrix bone properties contribute to bone fracture resistance.[241,242] Gourion-Arsiquaud et al. noted that alendronate and risedronate treatment in beagle dogs increased mineral content and matrix bone tissue maturity in cancellous and endochondral bone areas where bone turnover is higher.[243] These positive effects on bone properties were associated with a loss of 
bone heterogeneity that could contribute to increased brittleness and micro-crack accumulation. No significant difference was noted between low and high dose or between alendronate and risedronate treatments.

BP's effect on bone turnover is highly site specific.[244] In beagle dogs, 6 months of zoledronate treatment significantly suppressed intracortical bone remodeling in the mandible (99\%) compared to rib cortex (85\%) and tibia cortex (75\%).[244] These variations could by explained by the fact that mandible has a high bone turnover rate compared to other bones,[245] because of which BP's may get preferentially deposited in the mandible. In agreement with these findings, we noted that BP-treated mandibles were significantly harder than the femur in the present study. Furthermore, it is suggested that the rate of bone turnover suppression may vary according to the type of BP.[240]'[246] In an animal study, risedronate exhibited greater suppression of bone turnover earlier in the treatment compared to alendronate, which could be related to risedronate's superior potency on osteoclast inhibition and its lower affinity towards hydroxyapatite crystals.[240] Likewise, clinical studies have noted a greater degree of bone turnover suppression with highly potent intravenous BP's used in cancer patients.[246]

It is postulated that the jawbones, in comparison to other bones, are more prone to developing BRONJ lesions as they are subjected to constant stress from masticatory forces that cause microdamage. Microdamage in the form of microscopic cracks usually occurs in bone as result of physiologic stress.[247] Small cracks (30-80 mm) 
accumulate in the mineralized matrix of bone[247,248] in a mechanical load dependent manner.[237] Normally, physiologic bone remodeling repairs such microdamage. Suppressed bone remodeling caused by long-term BP therapy, however, may allow these microfractures and microdamage to accumulate and adversely affect the mechanical properties of bone. Accumulation of microdamage has been shown to reduce the strength of bones.[249]

An animal study evaluating cortical bone of dog rib reported that 1-year treatment with risedronate or alendronate suppressed intracortical bone remodeling by $57-68 \%$ and increased microdamage accumulation by 2 to 3 fold.[250] Hoefert et al. noted increased microfracture accumulation histologically in the jawbones of BRONJ patients when compared to control patients with osteomyelitis, osteoradionecrosis or osteoporosis (54\% vs 29\%).[251] Furthermore, an animal study by Li et al. demonstrated that BP treatment not only suppresses stochastic bone remodeling, but also suppresses the targeted repair of microdamaged bone.[252] This so-called microdamaged bone consists of brittle, adynamic and poorly vascularized bone that may eventually necrose and become symptomatic with secondary infection. This finding might explain why $40 \%$ of the BRONJ lesions occur spontaneously without an obvious traumatic event, such as tooth extraction.

Furthermore, recent studies have described atypical femoral fractures in osteoporosis patients receiving long-term alendronate therapy.[100,253] Such fragility fractures are otherwise rare, as the risk of proximal femur fracture under low-energy trauma is 
very small except in extremely osteoporotic bones. Over-suppression of bone turnover by long-term BP therapy may result in brittle bone with deteriorated mechanical properties that tends to fracture under low-energy distress.

In conclusion, our study indicates that long-term BP treatment results in increased bone hardness and brittleness. These outcomes are more significant within the rat mandible compared to femur, which may lead to increased microdamage accumulation within the mandible resulting in BRONJ development. 
Figure 1. Microhardness testing shows that trabecular and cortical bones from zoledronate-treated animals are significantly harder than the control group.
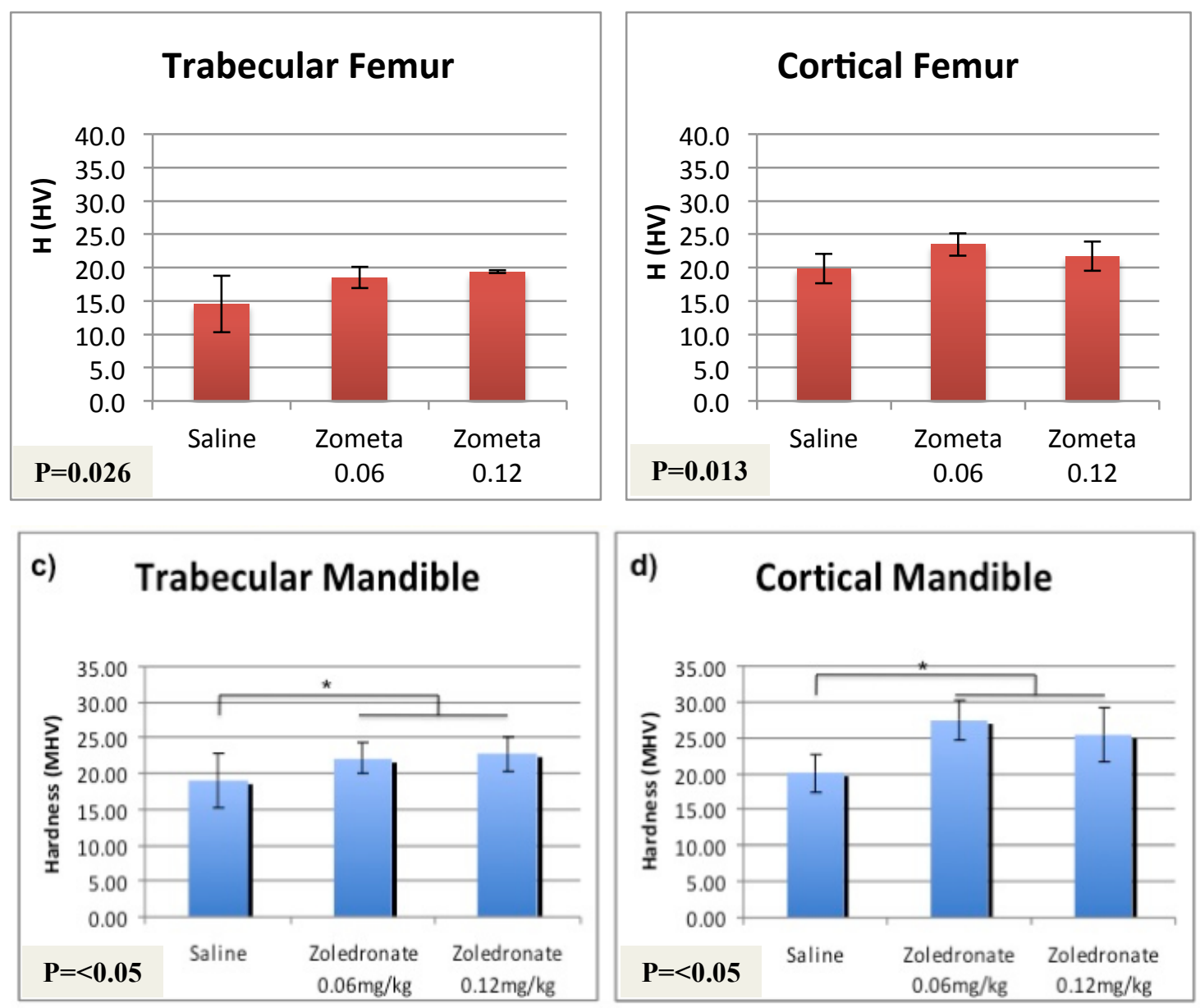
Figure 2. Microhardness testing shows that mandibles are harder than the femurs in the zoledronate treated animals, although the difference is statistically insignificant.

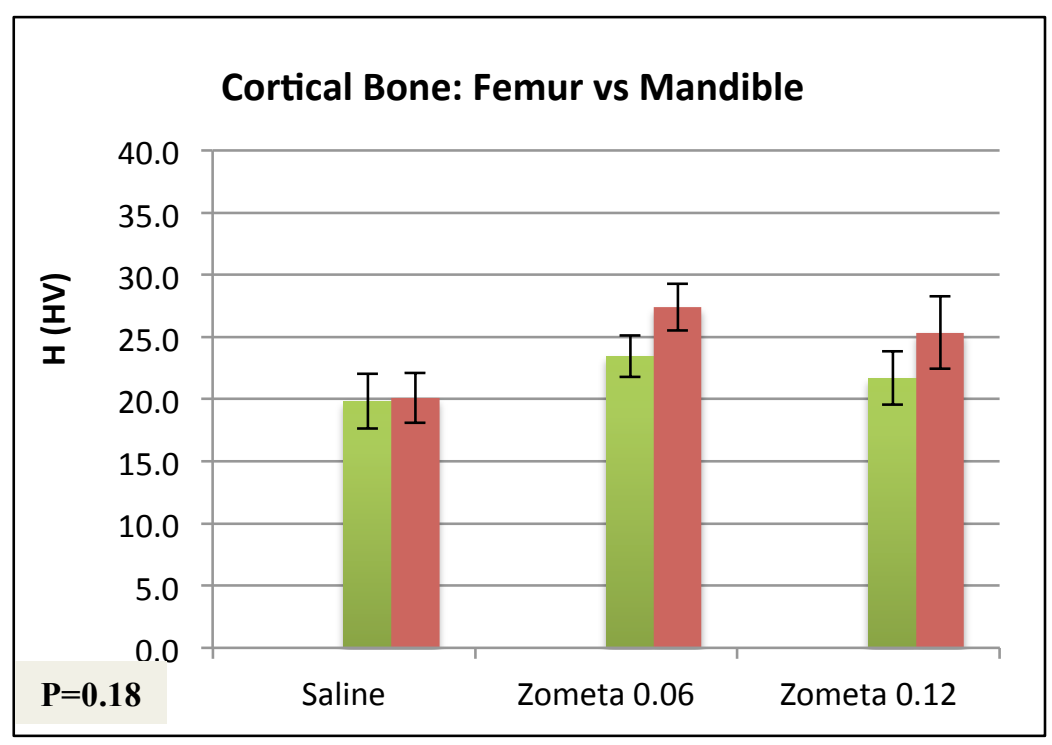

Femur

Mandible 
Figure 3. Microhardness testing shows that in the zoledronate-treated animals, the anterior region of the mandible is significantly harder than the posterior region.

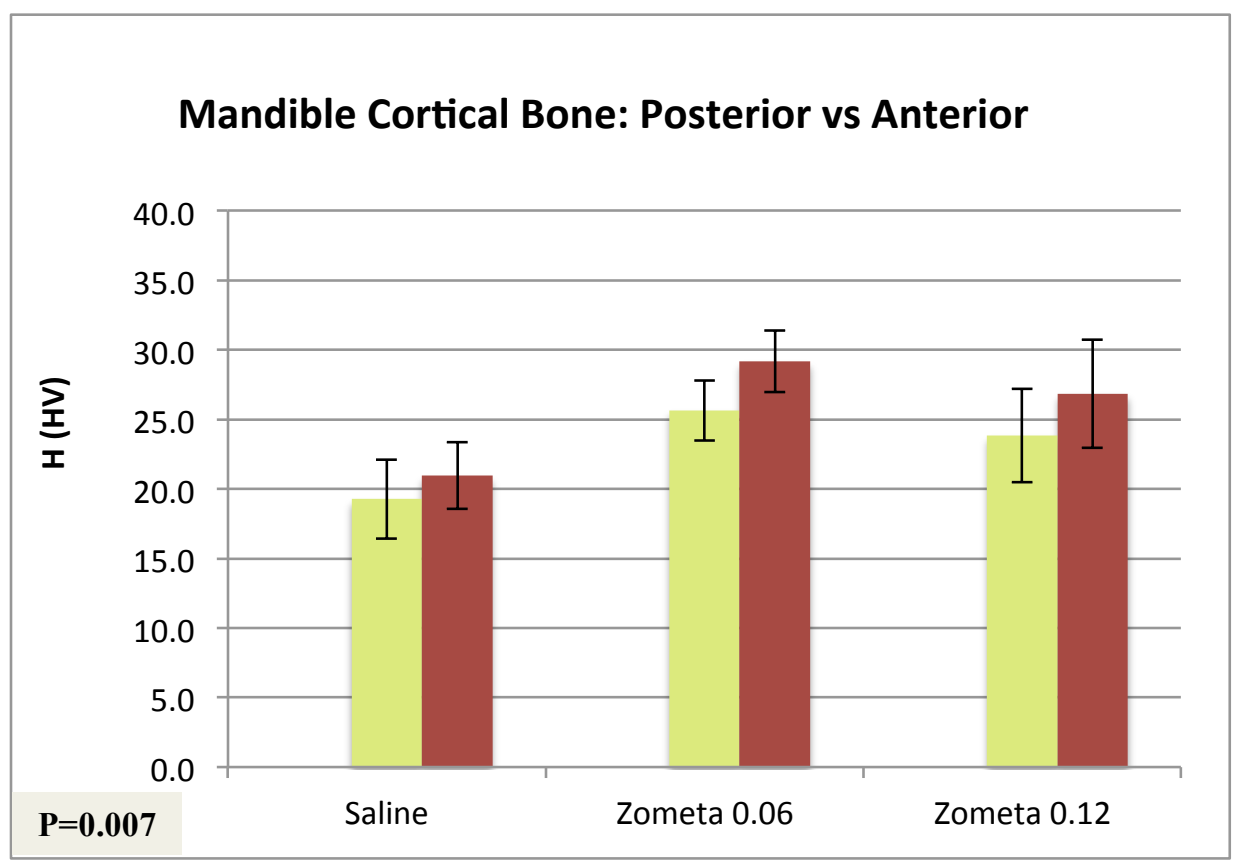

Posterior

Anterior 
Figure 4. SEM image shows higher Osteon Density in the two-zoledronate groups compared to the saline group.

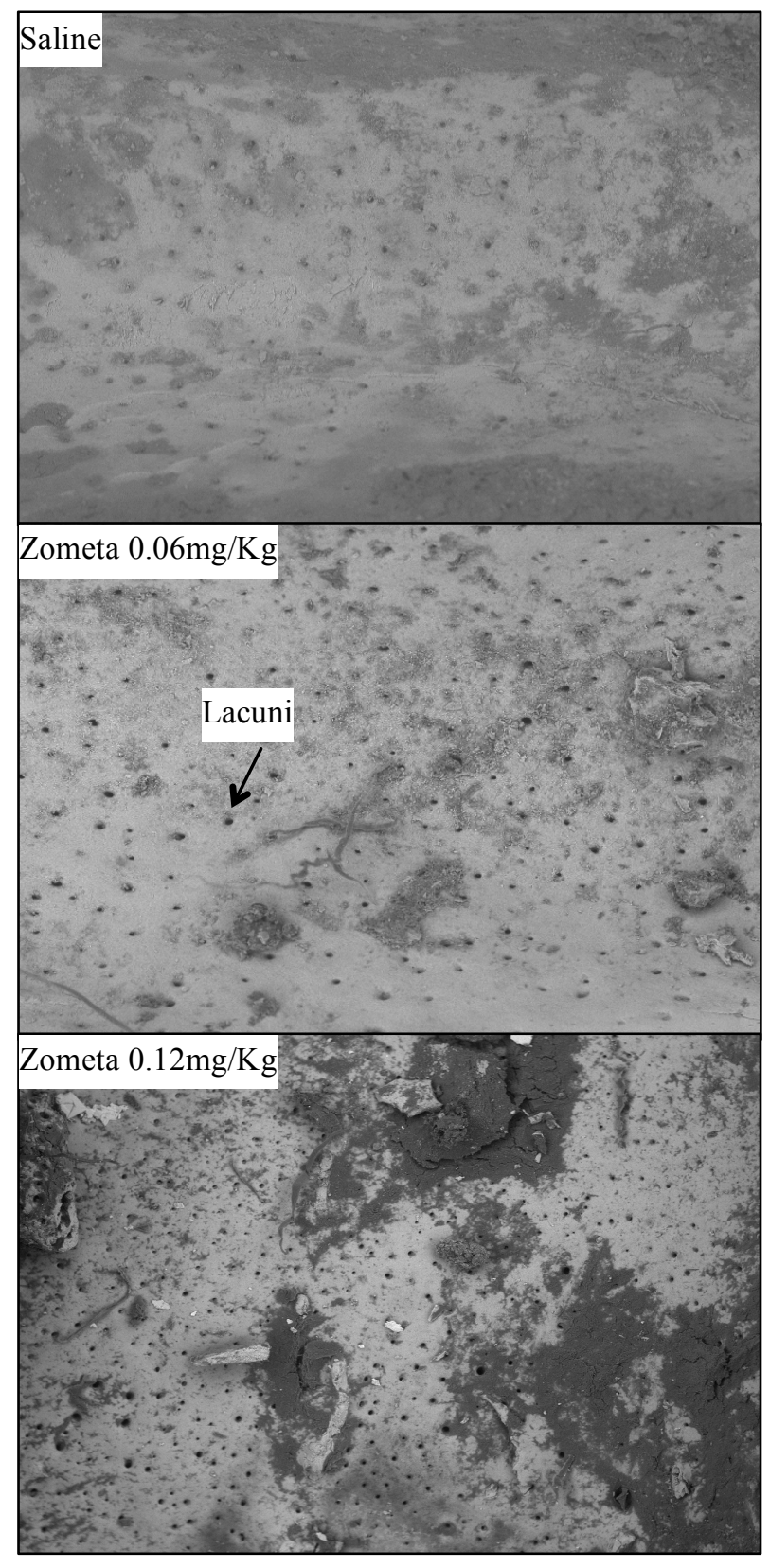


Figure 5. Histological image shows dense bone within the zoledronate group compared to the saline group ( $\mathrm{H} \& \mathrm{E}$, original magnification $\mathrm{x} 200)$.

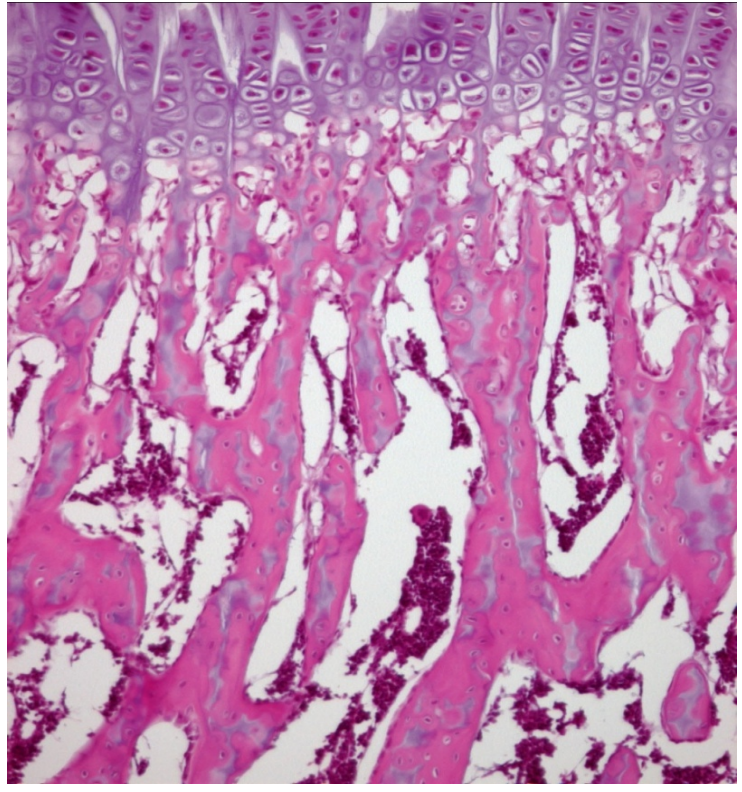

Saline

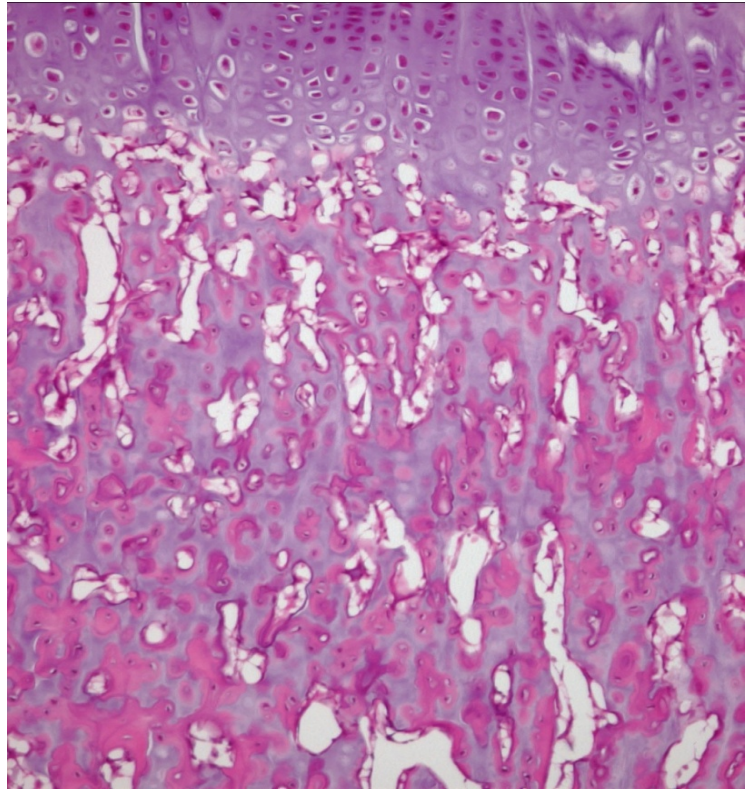

Zoledronate $0.06 \mathrm{mg} / \mathrm{kg}$ 


\section{CHAPTER 7}

\section{Conclusions and Discussion}


Previously published case reports have noted an increased incidence of BRONJ in patients receiving long-term BP therapy. Also, studies have noted a frequent history of dental infection, diabetes, and corticosteroid therapy among BRONJ subjects. In order to determine the frequency, etiology and risk factors that contribute to BRONJ pathogenesis, we undertook clinical and animal studies. Results from these epidemiologic/etiologic studies will significantly help towards the development of preventative and therapeutic modalities for BRONJ.

In this dissertation, we noted an overall BRONJ frequency of $3.1 \%(7.2 \%$ in multiple myeloma, $4.2 \%$ in breast cancer and $2.4 \%$ in prostate cancer). These percentages are similar to those reported in other studies where an overall BRONJ frequency of $\sim 5 \%$ (range $0.72 \%$ to $7.4 \%$ ) was noted in cancer patients treated with intravenous BP.[86,94,95,97] Hoff et al. in their analysis of 4019 patients reported an overall BRONJ frequency of 0.72\%.[97] The authors reported a higher BRONJ frequency in multiple myeloma patients $(2.4 \%)$ compared to breast cancer patients (1.2\%). Similarly, other studies have also reported a higher BRONJ frequency in multiple myeloma patients (ranging from $1.7 \%$ to $11 \%$ ) compared to breast cancer patients (ranging from $0.6 \%$ to $6.2 \%$ ).[86,87,95,97,111,129,130,131,132] The differences among numerous reported studies, including ours, are most probably due to variations in sample size, varying methods of data assessment/analysis and limitations due to voluntary case reporting. Also, previous studies indicate that BRONJ frequency may vary according to geographical locations reflecting the 
potential influence of genetic background and differences in oral health in the development of BRONJ.[86,94,95,97,111,129,130,131,132]

Previous studies have reported that the type of BP may play a role in BRONJ development, particularly the nitrogen-containing BP like pamidronate and zoledronate.[20,84,85,86,87,91,97,102,103,129] Bamias et al. and others have suggested that the risk is higher with zoledronate than pamidronate.[86,87,97,129] In agreement with these reports, we noted that the hazard of developing BRONJ was significantly higher with zoledronate infusions compared to pamidronate or pamidronate plus zoledronate infusions. The reason for this difference between the two types of BP is unknown. A plausible explanation is the more potent inhibitory effect on bone turnover and stronger anti-resorptive activity of zoledronate compared to pamidronate. Zoledronate is $10-100$ times more potent than pamidronate.[2] The significant impairment in bone turnover rate caused by continuous administration of zoledronate may eventually lead to increased bone fragility and in combination with other factors increase the risk of BRONJ development.

Our analysis demonstrated that BRONJ development was significantly associated with longer duration (months, $44.3 \pm 24.34$ vs $14.6 \pm 18.09, \mathrm{p}<0.001$ ) and increased number of BP infusions $(38.1 \pm 19.06$ vs 10.5 $\pm 12.81, \mathrm{p}<0.001)$ (Chapter 1, Table 2). We further confirmed by multivariate analysis that number of zoledronate infusions is an independent risk factor for BRONJ development. These results are consistent with previous studies that have noted that prolonged duration of $\mathrm{BP}$ exposure is a significant risk factor in the development of BRONJ.[86,87,97,102,129] 
In addition, BRONJ was more frequently noted in multiple myeloma and breast cancer patients compared to prostate cancer, lung cancer, renal cell carcinoma and other neoplasm group, although not statistically significant. The disparity in BRONJ incidence between multiple myeloma, breast cancer and other groups is most likely due to the differences in BP infusions and BP exposure time between these groups.

Stepwise Cox's proportional hazards regression analysis in the retrospective chart review study showed that diabetes, hypothyroidism, smoking, and number of zoledronate infusions was significantly associated with time to development of BRONJ (Chapter 1, Table 5). Most of these factors except hypothyroidism have previously reported to be associated with osteonecrosis development.[86,119,133]

The retrospective chart review study primarily focused on evaluating the frequency, risk factors and clinical presentation of BRONJ. The management of BRONJ patients and treatment outcomes are reported elsewhere.[128] Consistent with previous reports we found the mandible and posterior aspect of the jaws to be the most common site for BRONJ development. Our study also confirmed other published reports that tooth extraction is the most common inciting factor for BRONJ development. However, the risk of developing BRONJ as a consequence of dental extraction could not be further assessed due to the limited access to dental records of Non-BRONJ patients.

Previous reports suggest that poor oral hygiene predisposes patients to developing BRONJ. Our preliminary results also demonstrate BRONJ biopsy 
specimens to be infected with Actinomyces-like microorganisms. Furthermore, BRONJ lesions respond well to antibiotics and antimicrobial mouth rinses.[103,120] Collectively, these findings suggest that periodontal infection may play a role in BRONJ development. In order to test this hypothesis, we undertook a case-control clinical study investigating the role of periodontal disease in BRONJ development. We noted increased clinical attachment loss and decreased alveolar bone support in BRONJ subjects who had received intravenous BP therapy. Similar to our study, a retrospective study by Carmagnola et al. noted reduced alveolar bone support in BRONJ subjects compared to non-BRONJ subjects, although the difference was statistically insignificant.[151] A small sample size may have contributed to the insignificant differences in Carmagnola's study. In our case-control study we observed no significant difference in PI, GI, BOP and PD between BRONJ cases and controls. Imbalances in antibiotics and chlorhexidine mouth rinse usage between the groups may have eliminated or reduced the differences in clinical periodontal measurements. Lopez et al. noted that metronidazole plus amoxicillin therapy alone (without non-surgical or surgical therapy) reduced BOP by $26.5 \%$, mean PD by $0.85 \mathrm{~mm}$, and increased mean attachment gain by $0.3 \mathrm{~mm}$ in untreated chronic periodontitis patients.[152] Similar to Lopez's study, we noticed that CAL was less affected than PD with antibiotics use in BRONJ subjects, and bone height remained least affected. Resolving gingival inflammation alone with antibiotics use would decrease probe penetration and make CAL seem less severe. Antibiotics by themselves are expected to have least effect on alveolar bone height without additional interventions. 
In the case-control study, all subjects were cancer patients receiving multiple chemotherapy regimens and were above the mean age of 60 years. Both old age and chemotherapy-induced immunosuppression are risk indicators for periodontal disease.[153,154] In consistence with the previous reports we noted number of BP infusions to be a significant risk factor for BRONJ development.[86,97,155] We also noted tooth extraction to be the common inciting factor for BRONJ development. This is in agreement with other published reports that have frequently noted BRONJ following extraction of un-restorable teeth including teeth with active periodontal disease or periapical infection.[85,110,120,121] In the absence of effective bone resorption, conditions like tooth extraction and dental infection might result in tissue death, vascular loss, and eventually osteonecrosis. Furthermore, we noted BRONJ subjects had more missing teeth than controls. It is unclear if BRONJ patients in the present study had lost their teeth due to periodontal disease, dental caries or other factors.

Recently, a few studies have noted that preventive dental measures initiated before the start of BP treatment reduce the incidence of BRONJ by 3 to 7 fold in multiple myeloma and bone metastasis patients. $[156,157]$ In these studies, dental preventive measures included atraumatic tooth extraction, scaling/curettage, adjustment of ill-fitting denture, dental restorations, and endodontic therapy. Furthermore, a retrospective study by Montefusco et al. noted that prophylactic antibiotics reduced the incidence of BRONJ lesions after dental procedures.[158] 
These findings reinforce the notion that poor oral hygiene and dental infection may play a role in BRONJ development. The American Dental Association recommends that patients on antiresorptive therapy who have active periodontal disease should receive appropriate forms of nonsurgical periodontal therapy combined with periodontal re-evaluation every four to six weeks.[159],[92] The association further suggests that oral health program consisting of sound hygiene practices and regular dental care are valuable approaches for lowering risk of BRONJ development.[159],[92] Since tooth extractions constitute a risk factor for BRONJ, patients are advised to undergo regular dental examination and be treated with the aim of preventing progression of periodontal disease to the point where dental extractions are necessary. Furthermore, when possible, practitioners are advised to use atraumatic techniques to minimize dentoalveolar manipulation.[159],[92]

Current diagnostic tools to assess skeletal health in patients receiving BP therapy are limited to imaging techniques, including bone scans and magnetic resonance imaging (MRI) [160]. These methods rely on changes in the physical characteristics of bone, which minimizes the likelihood of identifying "at risk" patients before they develop clinical signs of BRONJ. Therefore, it is important to identify biomarkers that precede the onset of BRONJ lesions. Patient-based proteomic studies are valuable approaches in discovering informative biomarkers for various diseases. [161]. We utilized an advanced quantitative proteomics approach to identify differentially expressed salivary proteins in BRONJ patients that can serve as potential biomarkers for BRONJ diagnosis. This is the first study to identify 
differentially expressed salivary proteins in BRONJ patients that could serve as potential biomarkers for BRONJ diagnosis. Using a comprehensive and rigorous proteomic analysis, we identified 92 proteins that were significantly differentially expressed in BRONJ cases compared to controls. Many of the identified proteins require further scrutiny. As a preliminary step, we sought to examine MMP-9 and desmoplakin in the present study and found MMP-9 to be increased and desmoplakin to be decreased in BRONJ cases compared to controls. One or more of the differentially expressed proteins identified by this study may prove to be useful biomarkers for BRONJ diagnosis. The role of cell-junction-related proteins in BRONJ development needs to be further investigated.

It is hypothesized that long-term BP therapy may impair bone turnover and angiogenesis process leading to BRONJ development. Assessing bone turnover and angiogenesis markers appears to be valuable approaches for the early diagnosis and management of BRONJ. During bone remodeling, biochemical markers of bone turnover are released into circulation.[186] They comprise of enzymes/proteins secreted by osteoclasts and osteoblasts, degradation products formed during bone resorption, and precursors released during new bone formation. Similarly, during angiogenic process, various proteins including growth factors are released into circulation.[192] We aimed at serologically characterizing bone turnover, angiogenesis, endocrine, and inflammatory markers in BRONJ patients that could help provide insight into the pathogenesis of BRONJ development. We found no significant differences in bone turnover, endocrine, and inflammatory markers 
between controls and BRONJ cases. However, the mean VEGF values were significantly decreased in controls compared to BRONJ cases. It has been hypothesized that angiogenesis suppression by BP's might play an important role in BRONJ development. Previous studies have shown BP's to inhibit angiogenesis in bone and prostate tissues of murine models.[212,213] Similarly, clinical studies have shown BP's to inhibit angiogenesis and tumor cell bone invasion in cancer patients by reducing circulating levels of VEGF, basic fibroblast growth factor (bFGF), metalloproteinase 2 (MMP-2).[58,214] VEGF are not only important for endothelial cell proliferation, angiogenesis, and capillary permeability,[215] they also stimulate bone repair by promoting bone turnover.[216] Inactivation of VEGF has shown to decrease blood vessel invasion into growth plates associated with impaired trabecular bone formation,[217] decreased callus mineralization in femoral fractures, and subdued healing of tibial cortical bone defects.[216] These results are consistent with the finding that VEGF also plays a direct autocrine role in osteoblast differentiation.[216]

Recently, a clinical study by Vincenzi et al. reported that anti-angiogenic properties of $\mathrm{BP}$ are directly linked to BRONJ pathogenesis. Six of the 81 patients who developed BRONJ in this study (after a median number of 9 infusions), showed a significant decrease in circulating VEGF levels at day 7 and day 21 after the first BP administration. However, it is unclear if BRONJ patients in this study had concurrently received other anti-angiogenic agents for cancer treatment. An immunohistochemical study by Wehrhan et al. reported that angiogenesis, but not 
vascularization, was impaired in mucoperiosteal tissues adjacent to BRONJ lesions. The authors indicate that tissue regeneration in BRONJ patients may be impaired due to subdued vessel remodeling and neovessel formation.[218] Furthermore, a possible haplotype effect of VEGF polymorphism has been reported in female BRONJ patients.[219]

A few recent studies have also reported cases of exposed jaw bone, very similar to BRONJ, in patients treated with sunitinib and bevacizumab.[220,221,222,223,224] Both sunitinib and bevacizumab are antiangiogenic agents used in cancer treatment. Sunitinib is a tyrosine kinases inhibitor that targets receptors of VEGF, platelet-derived growth factor (PDGF), c-kit, FLT3 and RET.[225] Bevacizumab is a recombinant human monocloncal antibody that inhibits VEGF-A.[226] It is estimated that BRONJ frequency is higher in patients receiving combined $\mathrm{BP}$ and antiangiogenic treatment compared to patients receiving BP therapy alone.[220]

In the serum study, VEGF levels were significantly decreased in controls receiving BP therapy compared to BRONJ patients who had discontinued BP therapy for a mean period 11.4 months $(256 \pm 174$ vs $422 \pm 436 \mathrm{pg} / \mathrm{mL}, \mathrm{p}=0.02)$. BP's are known to reduce circulating VEGF levels in bone metastasis patients.[58,227] Although the precise mechanism is not completely understood, it is suggested that BP's may elicit several antiangiogenic-related cytokine patterns and cascades that can be probably mediated by the inhibition of isoprenylation of intracellular molecular 
targets (likely small GTP-binding proteins). Recent evidence indicate that zoledronate inhibit endothelial cell adhesion, migration and survival through the suppression of multiple, prenylation-dependent signaling pathways.[228,229] Kinases such as ERK1/2, JNK, Rock, FAK and PKB are affected by zoledronate in a prenylationdependent manner.[228] Collectively, these findings may explain the decreased serum VEGF levels noted in controls in the present study. However, the effect of discontinuation of BP therapy on VEGF levels is unknown. A previous study noted reversal of diminished VEGF-related glomerulonephritis following discontinuation of bevacizumab and zoledronate.[230] Since BP's have direct action on VEGF, discontinuation of BP therapy may increase VEGF levels, which explains the elevated VEGF levels seen in BRONJ cases compared to controls in the present study.

There was no wide difference in chemotherapeutic regimen between BRONJ cases and controls including antiangiogenic agents in the serum study. However, it is unknown if chemotherapy has confounding effects on angiogenesis markers.

Severe suppression of osteoclasts by long-term BP therapy may disrupt delicate balance between bone resorption and bone formation. It is hypothesized that long-term BP therapy may severely suppress bone turn over rate leading to impaired biomechanical properties of bone resulting in BRONJ development. Using an in vivo model, we studied changes in biomechanical properties within rat mandibles compared to femurs following long-term BP treatment. We found that long-term zoledronate treatment resulted in altered biomechanical properties. Long-term 
zoledronate treatment not only increased trabecular and cortical bone hardness, it also increased bone brittleness. These differences were more significant in the mandible compared to femur and were not dose dependent. The higher osteon density noted in zoledronate-treated bones may account for the above-mentioned biomechanical differences. Similar to our report, previous animal studies have noted altered bone mechanical properties with BP treatment.[238,239,240] Pozzi et al. noted that high dose zoledronate treatment increased bone mineral density, bone mineral content, trabecular bone volume and cortical bone thickness.[238] These effects were not dose dependent, suggesting that lower doses of zoledronate is adequate enough to inhibit bone resorption. In addition, mechanical testing revealed that increased bone stiffness was associated with increased bone brittleness, which is similar to our findings. Furthermore, it was noted that high doses of zoledronate interfere with both osteoblast and osteoclast lineages, resulting in possible inhibition of both cell types. It is postulated that the jawbones, in comparison to other bones, are more prone to developing BRONJ lesions as they are subjected to constant stress from masticatory forces that cause microdamage. Microdamage in the form of microscopic cracks usually occurs in bone as result of physiologic stress.[247] Small cracks (30-80 mm) accumulate in the mineralized matrix of bone[247,248] in a mechanical load dependent manner.[237] Normally, physiologic bone remodeling repairs such microdamage. Suppressed bone remodeling caused by long-term BP therapy, however, may allow these microfractures and microdamage to accumulate and adversely affect the mechanical properties of bone. Accumulation of microdamage has been shown to reduce the strength of bones.[249] In conclusion, our animal study 
indicates that long-term BP treatment results in increased bone hardness and brittleness. These outcomes are more significant within the rat mandible compared to femur, which may lead to increased microdamage accumulation within the mandible resulting in BRONJ development.

In conclusion, the results from this dissertation will help better understand BRONJ condition and provide the foundation required to initiate future intervention studies. 


\section{Supplementary Data}

Clinical features, risk factors, management and treatment outcomes of 26

\section{BRONJ patients}




\begin{abstract}
Purpose: To report clinical features, risk factors, management and treatment outcomes of nitrogen-containing bisphosphonate (n-BIS)-related osteonecrosis of the jaw (BRONJ).
\end{abstract}

Patients and Methods: Patients with suspected BRONJ were referred to the School of Dentistry for evaluation and management.

Results: Twenty-six patients ( 9 males, 17 females, mean age 64 years) were diagnosed with BRONJ. Twenty-three had received n-BIS therapy for cancer and 3 for osteoporosis. BRONJ lesions were noted more frequently in the mandible and in the posterior-sextants. Sixteen patients developed BRONJ following dentoalveolar procedures, while 10 developed it spontaneously. The mean time to develop BRONJ was shorter in cancer patients receiving intravenous n-BIS than osteoporosis patients receiving oral n-BIS (37.1 months versus 77.7 months, $\mathrm{P}=0.02)$. Based on the American Association of Oral and Maxillofacial Surgeons staging system, two patients were diagnosed with Stage I lesions, 19 with Stage II and 5 with Stage III lesions. Initial management of BRONJ was non-surgical, with debridement performed at subsequent visits if needed. BRONJ lesions healed completely in 4 patients, healed partially in 8 , remained stable in 7 , and progressed in 7 . Spontaneous lesions responded favorably to BRONJ management compared to lesions that developed following dentoalveolar procedures $(P=0.01)$. There was no significant difference in response to BRONJ management between patients who continued or discontinued n-BIS therapy after BRONJ diagnosis $(\mathrm{P}=0.54)$. 
Conclusion: Long-term n-BIS therapy and recent dental procedures are consistent findings in BRONJ patients. Spontaneous BRONJ lesions respond favorably to current BRONJ treatment strategies. 


\section{Introduction}

Nitrogen-containing bisphosphonates (n-BIS) are known inhibitors of bone resorption[254] and angiogenesis.[33,255] They are used in the treatment of various medical conditions including osteoporosis, multiple myeloma and breast cancer as they reduce bone pain, hypercalcemia and the risk of pathologic fractures. $[9,12,22,23,127]$ Prolonged n-BIS treatment is related with osteonecrosis of the jaws (BRONJ)[84,86,102,256], however, the pathophysiology of BRONJ remains unknown.

BRONJ lesions are defined as exposed necrotic bone in the maxillofacial area occurring in the absence of head and neck irradiation and showing no evidence of healing for at least 8 weeks after identification in patients who have had n-BIS treatment. BRONJ lesions can develop spontaneously or following dentoalveolar procedures. These lesions may be asymptomatic or present with pain, purulent discharge, swelling, tooth mobility and paresthesia.[102,256] Panoramic radiographs are usually inconclusive in early stages of BRONJ. Severe BRONJ with extensive bony involvement can appear as poorly defined radiolucent-radiopaque lesions. Computerized Tomography and Magnetic Resonance Imaging are effective in precisely determining the extension and characteristics of the lesion.[257] At present, there is no predictable remedy for BRONJ, therefore, patients are treated palliatively with antibiotics, antimicrobial mouth rinses and debridement of necrotic bone in an attempt to limit the extent of the condition. If the condition progresses to a state of 
recurrent infections, tooth loss and jaw fracture can occur further reducing the patient's quality of life.

Currently, there is a critical need to identify the risk factors for BRONJ which will help in developing effective prevention and treatment strategies. Here we describe our patient population and management outcomes of 26 BRONJ patients treated at the University of Minnesota School of Dentistry.

\section{Patients and Methods}

Over the last few years, an increasing number of patients were referred to the Division of Oral and Maxillofacial Surgery for evaluation and management of exposed bony lesions in the jaws. We established a program at the University of Minnesota School of Dentistry to monitor and evaluate all possible cases of BRONJ. IRB approval was obtained to retrospectively review charts of patients reporting to our institution between 2003 and 2007 for evaluation/management of exposed bone/osteomyelitis and with a history of n-BIS therapy. The patient's age, gender, medical history and dental history was abstracted from dental records. Information about n-BIS treatment and concurrent cancer treatment was obtained from the patient's medical records. 


\section{Eligibility Criteria:}

Inclusion criterion: All patients who had received at least one dose of n-BIS treatment within a year prior to developing exposed bone.

Exclusion criterion: Patients who received radiation therapy to the maxillofacial region or patients with a history of neoplastic disease directly involving the maxilla or mandible were excluded from this study.

\section{BRONJ Diagnosis:}

BRONJ diagnosis was based on clinical and radiographic findings, and biopsies were performed if metastatic disease had to be excluded. BRONJ was defined as follows: exposed bone in the maxillofacial area occurring in the absence of head and neck irradiation and showing no evidence of healing for at least 8 weeks after identification in patients who have had n-BIS treatment. This is consistent with the BRONJ working definition provided by scientific boards.[85,258]

\section{BRONJ Staging and Management:}

Patients were classified to Stage I, Stage II and Stage III groups according to the guidelines provided by the American Association of Oral and Maxillofacial Surgeons (AAOMS).[85] In brief, patients were classified to Stage I group if they had asymptomatic exposed bone without any evidence of infection. Patients exhibiting exposed necrotic bone with evidence of infection, pain and erythema, with or without purulence were classified to Stage II group. Patients were classified to Stage III group 
if they had exposed necrotic bone with evidence of pain, infection and one or more of the following: pathologic fracture, oral-cutaneous fistula or osteolysis extending to the inferior border.

\section{BRONJ Management Outcome:}

After initial BRONJ treatment, all patients were followed-up for more than 6 months. Based on changes in signs and symptoms and lesion size and number, we grouped patients into one of the following response categories: Complete response $(\mathrm{CR})$ complete re-growth of oral mucosa over previously exposed bone; Partial response (PR) - either a decrease in lesion size (largest linear dimension) or number of lesions and/or cessation of pain or signs of infection; Stable disease (SD) - no improvement in clinical signs or symptoms; or Progressive disease (PD) - increase in the size or the number of lesions or increased pain and severity of infection.

\section{Statistical Analysis:}

Duration of n-BIS exposure was defined as time in months from the date of first nBIS infusion administered to the last recorded infusion. Time to develop BRONJ was determined as time in months from the date of first n-BIS infusion administered to the date of BRONJ diagnosis. The chi-squared test was used for comparisons of proportions across levels of categorical variables. For continuous variables, the t test was used for comparison of means or medians. All analysis was performed using the R statistical software: A Language and Environment for Statistical Computing.[259] 


\section{Results}

Twenty-six patients were identified with BRONJ. Table 1 lists demographic, medical and n-BIS treatment characteristics of the study sample. Notably, 20 patients (77\%) had at least one of the following co-morbidities: diabetes mellitus, hypertension, hyperlipidemia, thromboembolism, or hypothyroidism.

BRONJ patients had received n-BIS for an average of $45.8 \pm 29.5$ months (Table 1). Notably, 24 patients had received $\geq 10$ intravenous infusions or oral doses of n-BIS before developing BRONJ. Nitrogen-containing bisphosphonate treatment was discontinued in 17 patients (65.4\%) within 2 months of BRONJ diagnosis, whereas, the remaining 9 patients (34.6\%) continued n-BIS treatment for an average of

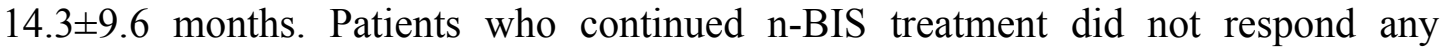
differently to BRONJ management than those who discontinued n-BIS therapy ( $\mathrm{P}=$ $0.54)$.

The mean time to develop BRONJ from the initiation of n-BIS therapy was $42.0 \pm 30.1$ months for all patients. The mean time to develop BRONJ, however, was shorter in cancer patients than osteoporosis patients $(37.1 \pm 26.0$ months versus $77.7 \pm 39.8$ months, $\mathrm{P}=0.02$ ).

\section{BRONJ Characteristics}

BRONJ characteristics are summarized in Table 2. Biopsy was performed in 7 patients $(27 \%)$ to exclude metastatic disease. Lesions were noted on bony exostoses 
in $5(45 \%)$ of the 11 patients who developed BRONJ spontaneously or following a restorative procedure. Overall, $80 \%$ of the spontaneous lesions were $\leq 1 \mathrm{~cm}$ in diameter. Just over half (56.3\%) of the lesions that developed following dentoalveolar procedures were $\leq 1 \mathrm{~cm}$ in diameter. Eight patients (31\%) had radiographic evidence of periodontitis, including widening of periodontal ligament spaces and loss of alveolar bone height. Seven patients (27\%) wore partial/complete dentures.

\section{BRONJ Management}

Management was directed towards alleviating pain, reducing lesion size, soft and hard tissue inflammation and/or infection. In most patients, initial treatment was nonsurgical and included antibiotics (penicillin, clindamycin, amoxicillin/clavulanate, and cephalexin), antifungal agents (Fluconazole, Nystatin), and rinsing or irrigating the lesion with an antimicrobial solution (chlorhexidine gluconate). Debridement without mucosal elevation was performed on 18 patients (69.2\%) at subsequent visits. Irrespective of the disease stage, loose segments of bony sequestra were removed. Patients were instructed to improve their oral hygiene and measures were taken to adjust any ill-fitting dentures if present. Four patients, in addition to antibiotics and debridement opted to undergo hyperbaric oxygen therapy.

\section{BRONJ Management Outcomes}

BRONJ management outcomes are summarized in Table 3. Both Stage I patients had lesions $\leq 1 \mathrm{~cm}$ in diameter that showed no evidence of progression (stable disease). Among the 19 stage II patients, $12(63.1 \%)$ patients had lesions $\leq 1 \mathrm{~cm}$ in diameter. 
Furthermore, in these 19 patients lesions completely resolved in $4(21 \%)$ and progressed in $5(26.3 \%)$. All 4 patients with complete responses had developed BRONJ spontaneously (i.e., not following a dentoalveolar procedure). Among the 5 Stage III patients, $4(80 \%)$ had lesions $\leq 1 \mathrm{~cm}$ in diameter. No Stage III patient exhibited a complete response, whereas the lesion progressed in 2 patients.

There was no significant difference in response to BRONJ management between Stage I, Stage II and Stage III patients or in patients with lesions $\leq 1 \mathrm{~cm}$ or $>1 \mathrm{~cm}$ in diameter $(\mathrm{P}=0.935)$. Patients with spontaneous lesions, however, responded significantly better to BRONJ management than patients who developed BRONJ lesions following dentoalveolar procedure (44.4\% complete response versus $0 \%$ complete response, $\mathrm{P}=0.01$, Table 3 )

One patient with extensive necrotic bone and pathologic fracture underwent segmental mandibulectomy and immediate reconstruction using a locking-screw reconstruction plate (Fig 1). Two months later the initial reconstruction failed, therefore, the reconstruction plate was removed and the right mandibular body was resected. Further mandibular reconstruction was not attempted. Thereafter, symptoms subsided with complete re-growth of oral mucosa over previously exposed bone. However, this patient was classified as having progressive disease because of permanent disability. All four patients who underwent hyperbaric oxygen therapy were initially classified to Stage II group. In these four patients, complete response 
was noted in one (Fig 2), partial response in two and progressive disease in the remaining 1 patient.

\section{Histology}

Bone specimens from 12 BRONJ patients were submitted for microscopic examination. The findings were consistent with a diagnosis of chronic osteomyelitis showing no evidence of malignancy (Fig 3). Necrotic areas revealed empty osteocyte lacunae with medullary spaces and surrounding osseous tissue colonized by Actinomyces-like microorganisms (Fig 3. A, C).

\section{Discussion}

Previous studies demonstrate that the cumulative hazard of developing BRONJ is significantly higher with zoledronate treatment compared to pamidronate or pamidronate plus zoledronate.[86,88] In consistent with these studies, we noted that most patients in our sample had received zoledronate only or zoledronate plus pamidronate. Furthermore, in these studies, cancer patients required a median number of 35 intravenous n-BIS infusions and median exposure of 39.3 months to develop BRONJ. In our study, cancer patients had received a mean number of 37.6 intravenous n-BIS infusions and were exposed to n-BIS for 40.2 months. In contrast to intravenous n-BIS, patients receiving oral n-BIS required significantly longer duration of exposure (mean, 82.0 months versus 45.8 months) and more drug doses (mean, 322 versus 37.6) to develop BRONJ $(\mathrm{P}=0.02)$. Nevertheless, some patients 
developed BRONJ soon after beginning n-BIS therapy. Two developed BRONJ within 8 months of starting n-BIS therapy and after receiving fewer than 10 infusions. There was no difference in response to BRONJ management between patients who continued or discontinued n-BIS therapy after BRONJ diagnosis.

In our study, BRONJ lesion occurred more frequently in the posterior lingual region of the mandible than maxilla, which is in agreement with previous reports.[102,256] Interestingly, $90 \%$ of the spontaneous lesions also occurred in the posterior mandible. These findings suggest that the posterior lingual portion of the mandible is more susceptible to BRONJ development. One of the plausible hypotheses is that thin mucosa, decreased vascularity and prominent exostoses that are often present in this area can be easily traumatized initiating osteonecrosis development. In support of this hypothesis, is a condition known as lingual mandibular sequestration, which occurs in apparently healthy individuals.[260]

In the present study, most patients developed BRONJ following dentoalveolar treatment, while some developed it without an obvious precipitating event. The most frequent dental procedures were tooth extraction followed by non-surgical root canal therapy. This data supports prior studies, which demonstrates that osseous manipulation is a risk factor for developing BRONJ.[256,261] For non-traumatic osteonecrosis, several etiologic events have been proposed including vascular occlusion, intravascular coagulation, inhibition of angiogenesis and mechanical stresses.[262] In our study, most patients had a history of hematologic, endocrine or 
vascular disorders (Table 1), which are associated with osteonecrosis[262], suggesting that systemic conditions could contribute towards BRONJ development. Nevertheless, patient's ability to respond to normal healing pattern needs to be evaluated in future studies.

Four patients underwent HBO therapy in addition to antibiotics, antimicrobial mouth rinses and necrotic bone debridement with varying outcomes. It is hypothesized that inhibition of angiogenesis leads to jaw necrosis. Since maxillary and mandibular alveolar bone has faster bone turnover rate compared to other bones, n-BIS may be highly concentrated in the jaws.[102] Coupled with anti-angiogenic properties, this anatomic concentration of n-BIS would make the alveolar bone more susceptible to BRONJ. Studies have evaluated the role of hyperbaric oxygen therapy (HBO) in increasing oxygenation of avascular tissues.[263,264] In this retrospective study, a limited number of BRONJ patients underwent HBO therapy, therefore, the efficacy of HBO could not be determined. A well-designed clinical trial is required to determine the effectiveness of HBO therapy.

Higher incidence of BRONJ has been reported in malignancies[20,84,86,88], particularly in multiple myeloma and breast cancer. Our study is in agreement with these reports, however, we noted more breast cancer patients $(42 \%)$ than multiple myeloma (27\%). This may be due to the retrospective nature of our study. In addition, the occurrence of BRONJ in osteoporosis implies that all patients using n-BIS are at risk of developing BRONJ and the risk ultimately depends on the route, frequency 
and the potency of n-BIS administered. Furthermore, the severity of cancer, and chemotherapy and corticosteroids use may modulate immunological status, increasing the risk of infection and impairing wound healing. Also, cancer patients receive up to 12-50 times higher doses of n-BIS compared to osteoporosis patients. A study by Hoff at al. reported that the development of BRONJ was associated with longer duration of malignant disease, longer duration of bone metastasis, which in turn relates to longer duration and greater cumulative doses of n-BIS therapy. ${ }^{[8]}$

Actinomyces spp has been proposed to play a role in BRONJ development, we therefore assessed 12 bone specimens for the presence of Actinomyces-like microorganisms. All bone samples had the presence of Actinomyces-like microorganisms. It is unclear if their presence contributes to BRONJ development or secondary infections of necrotic bone. A study by Hansen $\mathrm{T}$ et al. reported that Actinomyces was present in the majority of bone samples from both bisphosphonate related osteonecrosis and radiation induced osteonecrosis.[265] This suggests that chronically exposed maxillas or mandibles are colonized with Actinomyces ssp.

The retrospective nature of this preliminary study may have inherent bias and pose certain limitations, however, the results are in consistent with the previous large studies. It is one of the few studies to assess the association between BRONJ onsets, disease stage and lesion size with treatment outcome. In the present study we noticed that, spontaneous lesions are smaller in size and exhibit better response to BRONJ management. However, due to the small sample size, there were no statistically 
significant differences in response to BRONJ management between Stage I, Stage II and Stage III patients or in patients with lesions $\leq 1 \mathrm{~cm}$ or $>1 \mathrm{~cm}$ in diameter. A larger sample study would have the power to detect any true correlation if present. Nevertheless, results from the present study raise several hypotheses for future research. 
Table 1. Characteristics of BRONJ Patients $(\mathrm{N}=\mathbf{2 6})$

\begin{tabular}{|c|c|}
\hline \multicolumn{2}{|l|}{ Sex - no. (\%) } \\
\hline Male & $9(35.0)$ \\
\hline Female & $17(65.0)$ \\
\hline Age (mean \pm sd, in years) & $64 \pm 11$ \\
\hline \multicolumn{2}{|l|}{ Race or ethnic group - no. (\%) } \\
\hline White & $25(96.2)$ \\
\hline Black & $1(3.8)$ \\
\hline \multicolumn{2}{|c|}{ Pre-existing medical conditions - no. (\%) } \\
\hline Cancer & $23(88.0)$ \\
\hline Breast cancer & $11(42.0)$ \\
\hline Multiple Myeloma & $7(27.0)$ \\
\hline Prostate cancer & $3(11.5)$ \\
\hline Lung cancer & $1(3.8)$ \\
\hline Renal cell carcinoma & $1(3.8)$ \\
\hline Osteoporosis & $3(11.5)$ \\
\hline Diabetes mellitus & $5(19.2)$ \\
\hline Hypertension & $13(50.0)$ \\
\hline Hyperlipidemia & $6(23.0)$ \\
\hline Thromboembolism & $4(15.3)$ \\
\hline Hypothyroidism & $6(23.0)$ \\
\hline \multicolumn{2}{|l|}{ Environmental factors - no. (\%) } \\
\hline Smoking (> 10 years) & $7(27.0)$ \\
\hline Alcoholism & $4(15.3)$ \\
\hline \multicolumn{2}{|l|}{ Previous medication - no. (\%) } \\
\hline Antihypertensive ${ }^{*}$ & $13(50.0)$ \\
\hline Anticoagulants & $5(19.2)$ \\
\hline Chemotherapy $^{\dagger}$ & $23(88.4)$ \\
\hline Immunotherapy & $4(15.3)$ \\
\hline Corticosteroids ${ }^{\ddagger}$ & $16(61.5)$ \\
\hline \multicolumn{2}{|c|}{ Type of Bisphosphonates (n-BIS) - no. (\%) } \\
\hline \multicolumn{2}{|c|}{ Intravenous n-BIS } \\
\hline Zoledronate & $10(38.4)$ \\
\hline Pamidronate & $1(3.8)$ \\
\hline Pamidronate + Zoledronate & $10(38.4)$ \\
\hline Oral n-BIS ${ }^{\S}$ & $3(11.5)$ \\
\hline Intravenous + Oral n-BIS ${ }^{\rrbracket}$ & $2(7.7)$ \\
\hline \multicolumn{2}{|c|}{ Bisphosphonate doses (mean \pm s.d.) } \\
\hline Cancer + osteoporosis & $71.8 \pm 105.3$ \\
\hline Cancer & $37.6 \pm 23.3$ \\
\hline Breast cancer & $36.6 \pm 16.3$ \\
\hline Multiple myeloma & $45.7 \pm 30.9$ \\
\hline Prostate cancer & $36.0 \pm 30.3$ \\
\hline Osteoporosis & $322 \pm 144$ \\
\hline \multicolumn{2}{|c|}{ Duration of bisphosphonate exposure (in months) } \\
\hline Cancer + osteoporosis & $45.8 \pm 29.5$ \\
\hline Cancer & $40.2 \pm 25.0$ \\
\hline Breast cancer & $40.4 \pm 22.4$ \\
\hline Multiple myeloma & $52.4 \pm 33.7$ \\
\hline Prostate cancer & $28.7 \pm 16.3$ \\
\hline Osteoporosis & $82.0 \pm 34.1$ \\
\hline
\end{tabular}




\section{Table 1 Footnote.}

Plus-minus values are means $\pm \mathrm{SD}$

* Treatment consisted of Diuretics, $\beta$ blockers, Calcium channel blockers, and ACE inhibitors

${ }^{\dagger}$ All cancer patients had received one or more lines of chemotherapy treatment including Statins, Taxanes, Platinum group, Nitrogen mustards, and Thalidomide

$\$$ Pulsed corticosteroid treatment including dexamethasone or prednisone.

$\S$ Treatment included alendronate alone ( 2 patients) and alendrontae + ibandronate sequentially (1 patient)

『 Two patients received zoledronate + pamidronate + ibandronate sequentially 


\section{Table 2. BRONJ Characteristics}

\begin{tabular}{|ll|}
\hline Characteristics & $\begin{array}{l}\text { BRONJ } \\
(\mathbf{N}=\mathbf{2 6})\end{array}$ \\
Location - no. (\%) & \\
Maxilla & $4(15.4)$ \\
Mandible & $22(84.7)$ \\
Anterior sextant & $3(11.5)$ \\
Posterior sextant & $23(88.4)$ \\
BRONJ onset - no. (\%) & \\
Tooth extraction & $13(50.0)$ \\
Root canal treatment & $2(7.7)$ \\
Crown preparation & $1(3.8)$ \\
Spontaneous & $10(38.4)$ \\
Clinical findings - no. (\%) & \\
Pain & $21(80.7)$ \\
Infection & $18(69.0)$ \\
Purulent discharge & $14(53.8)$ \\
Facial swelling & $9(34.6)$ \\
Fistula & $3(11.5)$ \\
Paresthesia & $3(11.5)$ \\
Fracture & $2(7.7)$ \\
\hline
\end{tabular}




\section{Table 3. BRONJ Management outcome}

\begin{tabular}{|lcccc|}
\hline & $\begin{array}{c}\text { Complete } \\
\text { Response }\end{array}$ & $\begin{array}{c}\text { Partial } \\
\text { Response }\end{array}$ & $\begin{array}{c}\text { Stable } \\
\text { Disease }\end{array}$ & $\begin{array}{c}\text { Progressive } \\
\text { Disease }\end{array}$ \\
BRONJ stage - no. (\%) & & & & \\
Stage I $(\mathrm{n}=2)$ & $0(0.0)$ & $0(0.0)$ & $2(7.7)$ & $0(0.0)$ \\
Stage II $(\mathrm{n}=19)$ & $4(15.4)$ & $6(23.0)$ & $4(15.4)$ & $5(19.2)$ \\
Stage III $(\mathrm{n}=5)$ & $0(0.0)$ & $2(7.7)$ & $1(3.8)$ & $2(7.7)$ \\
Lesion size ${ }^{*}-$ no. $(\%)$ & & & & \\
$\quad$ Lesions $\leq 1 \mathrm{~cm}(\mathrm{n}=16)^{\dagger}$ & $3(18.7)$ & $5(31.2)$ & $4(25.0)$ & $4(23.5)$ \\
$\quad$ Lesions $>1 \mathrm{~cm}(\mathrm{n}=9)$ & $1(11.1)$ & $3(33.3)$ & $2(22.2)$ & $3(33.3)$ \\
BRONJ onset - no. $(\%)$ & & & & \\
$\quad$ Spontaneous lesions $(\mathrm{n}=9)^{\dagger}$ & $4(44.4)$ & $1(11.1)$ & $2(22.2)$ & $2(22.2)$ \\
Dento-alveolar procedure $(\mathrm{n}=16)^{*}$ & $0(0.0)$ & $7(43.7)$ & $4(25.0)$ & $5(31.2)$ \\
\hline
\end{tabular}

\footnotetext{
* Lesion size measured as largest diameter

${ }^{\dagger}$ Missing data for one subject

$\$$ Lesions developing following a dentoalveolar procedure

Complete response (CR), Partial response (PR), Stable disease (SD) and Progressive disease (PD) (see text for details)
} 
Figure 1.

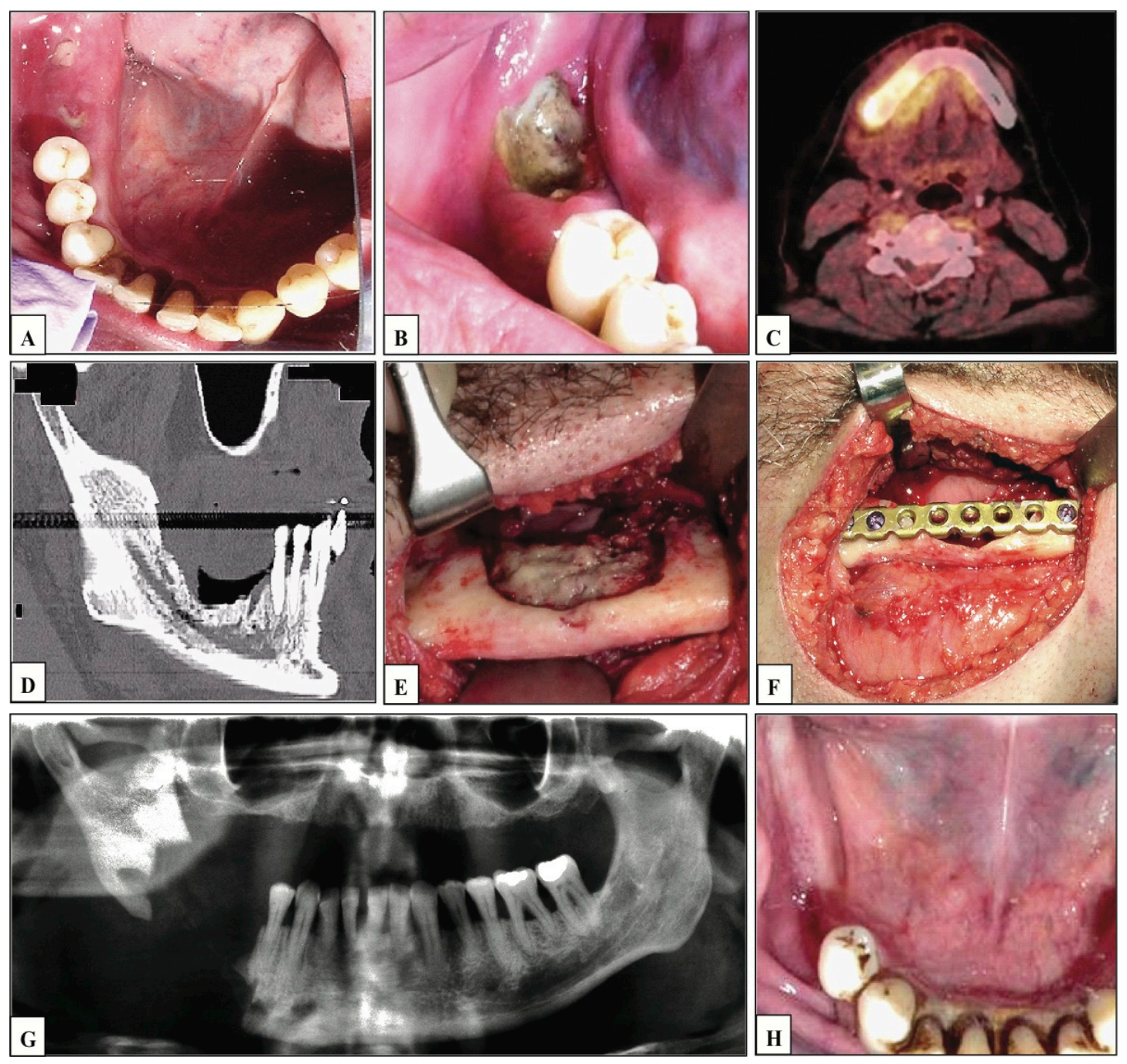


Fig 1. (A) Non-healing extraction sites (30, 31) with exposed alveolar bone $(0.5 \mathrm{~cm} x 0.5 \mathrm{~cm})$. (B) Note the rapid progression of the lesion $(1.5 \mathrm{~cm} \times 2 \mathrm{~cm})$. (C) Axial positron emission tomography - computed tomography (PET-CT) image shows diffuse, intense hypermetabolic activity. (D) CT scan revealed an extensive area of involved bone, which was clinically not apparent. (E) Necrotic bone was resected through a submandibular approach. (F) Immediate reconstruction using a reconstruction plate. (G) Orthopantomogram after the primary reconstruction failed, showing the right mandibular body resected without the reconstruction plate. (H) Later, there was complete re-growth of oral mucosa over previously exposed bone. Note: This patient had following medical history - diabetes, hyperlipidemia, hypertension, renal cell carcinoma, and longer duration of n-BIS therapy (26 months). 
Figure 2.

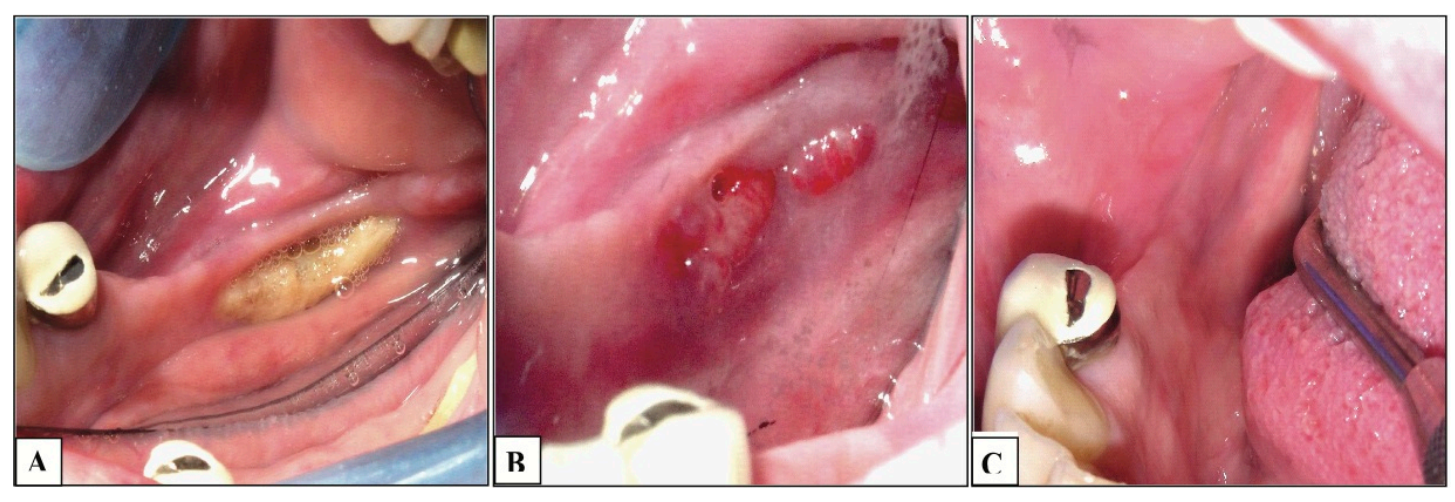

Fig 2. (A) Spontaneous bone exposure $(0.05 \mathrm{~cm} \times 0.05 \mathrm{~cm})$ in the lingual region of posterior right mandible. (B) Post-debridement picture shows evidence of granulation tissue. (C) Following systemic antibiotic and antimicrobial mouth rinsing, and hyperbaric oxygen therapy (30 sessions 2.4 ATA for $90 \mathrm{mins}$ ), there was complete regrowth of oral mucosa over previously exposed bone with cessation of pain. 
Figure 3.
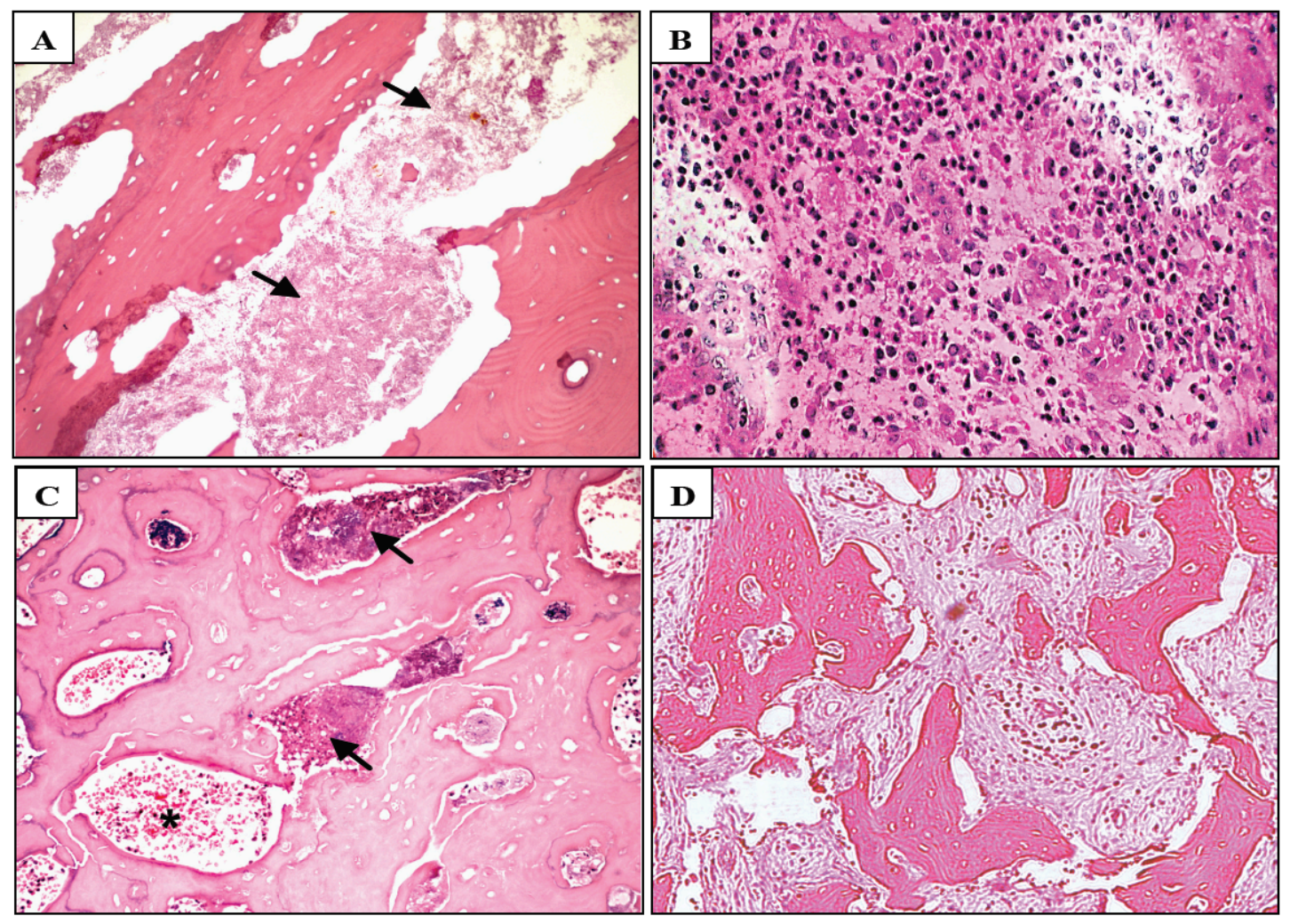

Figure 3. (a) Photomicrograph of necrotic bone shows empty lacunae and clusters of microorganisms (arrow) diffusely filling medullary space ( $\mathrm{H} \& \mathrm{E}$, original magnification $\mathrm{x}$ 100). (b) Note the presence of inflammatory cells and granulation tissue (H \& E, original magnification x 200). (c) Photomicrograph of tissue specimen shows medullary space filled with clusters of microorganisms (arrow) and inflammatory infiltrate (asterisk) ( $\mathrm{H} \& \mathrm{E}$, original magnification $\mathrm{x} 100)$. (d) Photomicrograph of necrotic bone shows empty lacunae $(\mathrm{H} \& \mathrm{E}$, original magnification $\mathrm{x}$ 100) 


\section{References}

1. Jung A, Bisaz S, Fleisch H (1973) The binding of pyrophosphate and two diphosphonates by hydroxyapatite crystals. Calcif Tissue Res 11: 269-280.

2. Fleisch H (1998) Bisphosphonates: mechanisms of action. Endocr Rev 19: 80-100.

3. Russell RG, Croucher PI, Rogers MJ (1999) Bisphosphonates: pharmacology, mechanisms of action and clinical uses. Osteoporos Int 9 Suppl 2: S66-80.

4. Rogers MJ, Gordon S, Benford HL, Coxon FP, Luckman SP, et al. (2000) Cellular and molecular mechanisms of action of bisphosphonates. Cancer 88: 29612978.

5. Fleisch H (1969) Diphosphonates inhibit hydroxyapatite dissolution in vitro and bone resorption in tissue culture and in vivo. Science 165: 1262-1264.

6. Russell RG (1970) The influence of pyrophosphate, condensed phosphates, phosphonates and other phosphate compounds on the dissolution of hydroxyapatite in vitro and on bone resorption induced by parathyroid hormone in tissue culture and in thyroparathyroidectomised rats. Calcif Tissue Res 6: 183-196.

7. Francis MD, Russell RG, Fleisch H (1969) Diphosphonates inhibit formation of calcium phosphate crystals in vitro and pathological calcification in vivo. Science 165: 1264-1266.

8. National Osteoporosis Foundation.

9. Liberman UA, Weiss SR, Broll J, Minne HW, Quan H, et al. (1995) Effect of oral alendronate on bone mineral density and the incidence of fractures in postmenopausal osteoporosis. The Alendronate Phase III Osteoporosis Treatment Study Group. N Engl J Med 333: 1437-1443.

10. Bone HG, Hosking D, Devogelaer JP, Tucci JR, Emkey RD, et al. (2004) Ten years' experience with alendronate for osteoporosis in postmenopausal women. The New England journal of medicine 350: 1189-1199.

11. Hochberg MC, Greenspan S, Wasnich RD, Miller P, Thompson DE, et al. (2002) Changes in bone density and turnover explain the reductions in incidence of nonvertebral fractures that occur during treatment with antiresorptive agents. J Clin Endocrinol Metab 87: 1586-1592.

12. Black DM, Cummings SR, Karpf DB, Cauley JA, Thompson DE, et al. (1996) Randomised trial of effect of alendronate on risk of fracture in women with existing vertebral fractures. Fracture Intervention Trial Research Group. Lancet 348: 1535-1541.

13. Marketos M (2004) The top 200 brand drugs in 2003 (by units). Drug Topics. pp. 148:176.

14. Black DM (1996) Randomised trial of effect of alendronate on risk of fracture in women with existing vertebral fractures. Fracture Intervention Trial Research Group. Lancet 348: 1535-1541.

15. Black DM, Delmas PD, Eastell R, Reid IR, Boonen S, et al. (2007) Once-yearly zoledronic acid for treatment of postmenopausal osteoporosis. N Engl J Med 356: 1809-1822. 
16. Diel IJ, Solomayer EF, Bastert G (2000) Bisphosphonates and the prevention of metastasis: first evidences from preclinical and clinical studies. Cancer 88: 3080-3088.

17. Senaratne SG, Pirianov G, Mansi JL, Arnett TR, Colston KW (2000) Bisphosphonates induce apoptosis in human breast cancer cell lines. $\mathrm{Br} \mathrm{J}$ Cancer 82: 1459-1468.

18. Tassone P, Forciniti S, Galea E, Morrone G, Turco MC, et al. (2000) Growth inhibition and synergistic induction of apoptosis by zoledronate and dexamethasone in human myeloma cell lines. Leukemia : official journal of the Leukemia Society of America, Leukemia Research Fund, UK 14: 841844.

19. Reid IR (2005) Comparison of a single infusion of zoledronic acid with risedronate for Paget's disease. The New England journal of medicine 353: 898-908.

20. Woo SB, Hellstein JW, Kalmar JR (2006) Narrative [corrected] review: bisphosphonates and osteonecrosis of the jaws. Ann Intern Med 144: 753-761.

21. Ezra A, Golomb G (2000) Administration routes and delivery systems of bisphosphonates for the treatment of bone resorption. Adv Drug Deliv Rev 42: 175-195.

22. Berenson JR, Hillner BE, Kyle RA, Anderson K, Lipton A, et al. (2002) American Society of Clinical Oncology clinical practice guidelines: the role of bisphosphonates in multiple myeloma. J Clin Oncol 20: 3719-3736.

23. Hillner BE, Ingle JN, Chlebowski RT, Gralow J, Yee GC, et al. (2003) American Society of Clinical Oncology 2003 update on the role of bisphosphonates and bone health issues in women with breast cancer. J Clin Oncol 21: 4042-4057.

24. Sato M (1991) Bisphosphonate action: alendronate localization in rat bone osteoclast ultrastructure. The Journal of clinical investigation 88: 2095-2105.

25. Masarachia P, Weinreb M, Balena R, Rodan GA (1996) Comparison of the distribution of $3 \mathrm{H}$-alendronate and $3 \mathrm{H}$-etidronate in rat and mouse bones. Bone 19: 281-290.

26. Lin JH (1996) Bisphosphonates: A review of their pharmacokinetic properties. Bone 18: 75-85.

27. Flanagan AM, Chambers TJ (1991) Inhibition of bone resorption by bisphosphonates: interactions between bisphosphonates, osteoclasts, and bone. Calcif Tissue Int 49: 407-415.

28. Boonekamp PM, van der Wee-Pals LJ, van Wijk-van Lennep MM, Thesing CW, Bijvoet OL (1986) Two modes of action of bisphosphonates on osteoclastic resorption of mineralized matrix. Bone Miner 1:27-39.

29. Colucci S, Minielli V, Zambonin G, Cirulli N, Mori G, et al. (1998) Alendronate reduces adhesion of human osteoclast-like cells to bone and bone proteincoated surfaces. Calcif Tissue Int 63: 230-235.

30. Piper K, Boyde A, Jones SJ (1994) The effect of 3-amino-1-hydroxypropylidene1,1-bisphosphonate (ADP) on the resorptive function of osteoclasts of known nuclear number. Calcif Tissue Int 54: 56-61. 
31. Hughes DE, Wright KR, Uy HL, Sasaki A, Yoneda T, et al. (1995) Bisphosphonates promote apoptosis in murine osteoclasts in vitro and in vivo. J Bone Miner Res 10: 1478-1487.

32. Reszka AA, Halasy-Nagy JM, Masarachia PJ, Rodan GA (1999) Bisphosphonates act directly on the osteoclast to induce caspase cleavage of mst1 kinase during apoptosis. A link between inhibition of the mevalonate pathway and regulation of an apoptosis-promoting kinase. J Biol Chem 274: 34967-34973.

33. Fournier P, Boissier S, Filleur S, Guglielmi J, Cabon F, et al. (2002) Bisphosphonates inhibit angiogenesis in vitro and testosterone-stimulated vascular regrowth in the ventral prostate in castrated rats. Cancer Res 62: 6538-6544.

34. Ledoux D, Hamma-Kourbali Y, Di Benedetto M, Foucault-Bertaud A, Oudar O, et al. (2006) A new dimethyl ester bisphosphonate inhibits angiogenesis and growth of human epidermoid carcinoma xenograft in nude mice. Anticancer Drugs 17: 479-485.

35. Rogers MJ, Watts DJ, Russell RG (1997) Overview of bisphosphonates. Cancer 80: 1652-1660.

36. Murakami H, Takahashi N, Sasaki T, Udagawa N, Tanaka S, et al. (1995) A possible mechanism of the specific action of bisphosphonates on osteoclasts: tiludronate preferentially affects polarized osteoclasts having ruffled borders. Bone 17: 137-144.

37. Sato M, Grasser W (1990) Effects of bisphosphonates on isolated rat osteoclasts as examined by reflected light microscopy. Journal of bone and mineral research : the official journal of the American Society for Bone and Mineral Research 5: 31-40.

38. Miller SC, Jee WS (1979) The effect of dichloromethylene diphosphonate, a pyrophosphate analog, on bone and bone cell structure in the growing rat. The Anatomical record 193: 439-462.

39. Selander K, Lehenkari P, Vaananen HK (1994) The effects of bisphosphonates on the resorption cycle of isolated osteoclasts. Calcif Tissue Int 55: 368-375.

40. Hiroi-Furuya E, Kameda T, Hiura K, Mano H, Miyazawa K, et al. (1999) Etidronate (EHDP) inhibits osteoclastic-bone resorption, promotes apoptosis and disrupts actin rings in isolate-mature osteoclasts. Calcif Tissue Int 64: 219-223.

41. Lerner UH, Larsson A (1987) Effects of four bisphosphonates on bone resorption, lysosomal enzyme release, protein synthesis and mitotic activities in mouse calvarial bones in vitro. Bone 8: 179-189.

42. Carano A, Teitelbaum SL, Konsek JD, Schlesinger PH, Blair HC (1990) Bisphosphonates directly inhibit the bone resorption activity of isolated avian osteoclasts in vitro. The Journal of clinical investigation 85: 456-461.

43. Boonekamp PM, Lowik CW, van der Wee-Pals LJ, van Wijk-van Lennep ML, Bijvoet OL (1987) Enhancement of the inhibitory action of APD on the transformation of osteoclast precursors into resorbing cells after dimethylation of the amino group. Bone Miner 2: 29-42. 
44. Hughes DE, MacDonald BR, Russell RG, Gowen M (1989) Inhibition of osteoclast-like cell formation by bisphosphonates in long-term cultures of human bone marrow. The Journal of clinical investigation 83: 1930-1935.

45. Fast DK, Felix R, Dowse C, Neuman WF, Fleisch H (1978) The effects of diphosphonates on the growth and glycolysis of connective-tissue cells in culture. The Biochemical journal 172: 97-107.

46. Guenther HL, Guenther HE, Fleisch H (1979) Effects of 1-hydroxyethane-1,1diphosphonate and dichloromethanediphosphonate on rabbit articular chondrocytes in culture. The Biochemical journal 184: 203-214.

47. Sahni M, Guenther HL, Fleisch H, Collin P, Martin TJ (1993) Bisphosphonates act on rat bone resorption through the mediation of osteoblasts. The Journal of clinical investigation 91: 2004-2011.

48. Frith JC, Monkkonen J, Auriola S, Monkkonen H, Rogers MJ (2001) The molecular mechanism of action of the antiresorptive and antiinflammatory drug clodronate: evidence for the formation in vivo of a metabolite that inhibits bone resorption and causes osteoclast and macrophage apoptosis. Arthritis Rheum 44: 2201-2210.

49. Roelofs AJ, Thompson K, Gordon S, Rogers MJ (2006) Molecular mechanisms of action of bisphosphonates: current status. Clin Cancer Res 12: 6222s-6230s.

50. Amin D, Cornell SA, Perrone MH, Bilder GE (1996) 1-Hydroxy-3(methylpentylamino)-propylidene-1,1-bisphosphonic acid as a potent inhibitor of squalene synthase. Arzneimittel-Forschung 46: 759-762.

51. Amin D, Cornell SA, Gustafson SK, Needle SJ, Ullrich JW, et al. (1992) Bisphosphonates used for the treatment of bone disorders inhibit squalene synthase and cholesterol biosynthesis. Journal of lipid research 33: 16571663.

52. Gibbs JB, Oliff A (1997) The potential of farnesyltransferase inhibitors as cancer chemotherapeutics. Annual review of pharmacology and toxicology 37: 143166.

53. Clark EA, King WG, Brugge JS, Symons M, Hynes RO (1998) Integrin-mediated signals regulated by members of the rho family of GTPases. The Journal of cell biology 142: 573-586.

54. Ridley AJ, Hall A (1992) The small GTP-binding protein rho regulates the assembly of focal adhesions and actin stress fibers in response to growth factors. Cell 70: 389-399.

55. Ridley AJ, Paterson HF, Johnston CL, Diekmann D, Hall A (1992) The small GTP-binding protein rac regulates growth factor-induced membrane ruffling. Cell 70: 401-410.

56. Luckman SP, Hughes DE, Coxon FP, Graham R, Russell G, et al. (1998) Nitrogen-containing bisphosphonates inhibit the mevalonate pathway and prevent post-translational prenylation of GTP-binding proteins, including Ras. Journal of bone and mineral research : the official journal of the American Society for Bone and Mineral Research 13: 581-589.

57. Russell RG, Watts NB, Ebetino FH, Rogers MJ (2008) Mechanisms of action of bisphosphonates: similarities and differences and their potential influence on clinical efficacy. Osteoporosis international : a journal established as result of 
cooperation between the European Foundation for Osteoporosis and the National Osteoporosis Foundation of the USA 19: 733-759.

58. Santini D (2002) Pamidronate induces modifications of circulating angiogenic factors in cancer patients. Clinical cancer research : an official journal of the American Association for Cancer Research 8: 1080-1084.

59. Vincenzi B, Santini D, Rocci L, Tonini G (2003) Bisphosphonates: new antiangiogenic molecules in cancer treatment? Annals of oncology : official journal of the European Society for Medical Oncology / ESMO 14: 806-807.

60. J. Wood, C. Schnell, Green JR (2000) Zoledronic Acid (Zometa), a Potent Inhibitor of Bone Resorption, Inhibits Proliferation and Induces Apoptosis in Human Endothelial Cells in Vitro and Is Anti-Angiogenic in a Murine Growth Factor Implant Model. Proc Am Soc Clin Oncol 664: 664-665.

61. Kapitola J, Zak J (1998) Effect of pamidronate on bone blood flow in oophorectomized rats. Physiol Res 47: 237-240.

62. Wood J, Bonjean K, Ruetz S, Bellahcene A, Devy L, et al. (2002) Novel antiangiogenic effects of the bisphosphonate compound zoledronic acid. J Pharmacol Exp Ther 302: 1055-1061.

63. Cackowski FC, Anderson JL, Patrene KD, Choksi RJ, Shapiro SD, et al. (2010) Osteoclasts are important for bone angiogenesis. Blood 115: 140-149.

64. Schoutens A VM, L Hermite M, Tricot A, Verschaeren A, Dourov N, Heilporn A (1984) Increase of bone blood flow, an initial step of bone demineralization in the rat. Calcif Tiss Int 36: 53.

65. Conte P, Guarneri V (2004) Safety of intravenous and oral bisphosphonates and compliance with dosing regimens. Oncologist 9 Suppl 4: 28-37.

66. Strampel W, Emkey R, Civitelli R (2007) Safety considerations with bisphosphonates for the treatment of osteoporosis. Drug safety : an international journal of medical toxicology and drug experience 30: 755-763.

67. Adami S, Zamberlan N (1996) Adverse effects of bisphosphonates. A comparative review. Drug safety : an international journal of medical toxicology and drug experience 14: 158-170.

68. Hewitt RE, Lissina A, Green AE, Slay ES, Price DA, et al. (2005) The bisphosphonate acute phase response: rapid and copious production of proinflammatory cytokines by peripheral blood gd $\mathrm{T}$ cells in response to aminobisphosphonates is inhibited by statins. Clin Exp Immunol 139: 101111.

69. Markowitz GS (2003) Toxic acute tubular necrosis following treatment with zoledronate (Zometa). Kidney Int 64: 281-289.

70. Bounameaux HM, Schifferli J, Montani JP, Jung A, Chatelanat F (1983) Renal failure associated with intravenous diphosphonates. Lancet 1: 471.

71. Black DM (2007) Once-yearly zoledronic acid for treatment of postmenopausal osteoporosis. The New England journal of medicine 356: 1809-1822.

72. Cummings SR, Schwartz AV, Black DM (2007) Alendronate and atrial fibrillation. The New England journal of medicine 356: 1895-1896.

73. Heckbert SR, Li G, Cummings SR, Smith NL, Psaty BM (2008) Use of alendronate and risk of incident atrial fibrillation in women. Archives of internal medicine 168: 826-831. 
74. Aviles RJ, Martin DO, Apperson-Hansen C, Houghtaling PL, Rautaharju P, et al. (2003) Inflammation as a risk factor for atrial fibrillation. Circulation 108: 3006-3010.

75. Boos CJ, Anderson RA, Lip GY (2006) Is atrial fibrillation an inflammatory disorder? European heart journal 27: 136-149.

76. Dicuonzo G, Vincenzi B, Santini D, Avvisati G, Rocci L, et al. (2003) Fever after zoledronic acid administration is due to increase in TNF-alpha and IL-6. Journal of interferon \& cytokine research : the official journal of the International Society for Interferon and Cytokine Research 23: 649-654.

77. Marx RE (2008) Uncovering the cause of "phossy jaw" Circa 1858 to 1906: oral and maxillofacial surgery closed case files-case closed. Journal of oral and maxillofacial surgery : official journal of the American Association of Oral and Maxillofacial Surgeons 66: 2356-2363.

78. Miles AE (1972) Phosphorus necrosis of the jaw: 'phossy jaw'. British dental journal 133: 203-206.

79. Demerjian N, Bolla G, Spreux A (1999) Severe oral ulcerations induced by alendronate. Clinical rheumatology 18: 349-350.

80. de Groen PC, Lubbe DF, Hirsch LJ, Daifotis A, Stephenson W, et al. (1996) Esophagitis associated with the use of alendronate. The New England journal of medicine 335: 1016-1021.

81. Marx RE (2003) Pamidronate (Aredia) and zoledronate (Zometa) induced avascular necrosis of the jaws: a growing epidemic. Journal of oral and maxillofacial surgery : official journal of the American Association of Oral and Maxillofacial Surgeons 61: 1115-1117.

82. Migliorati CA (2003) Bisphosphanates and oral cavity avascular bone necrosis. Journal of clinical oncology : official journal of the American Society of Clinical Oncology 21: 4253-4254.

83. Wang J, Goodger NM, Pogrel MA (2003) Osteonecrosis of the jaws associated with cancer chemotherapy. Journal of oral and maxillofacial surgery : official journal of the American Association of Oral and Maxillofacial Surgeons 61: 1104-1107.

84. Dunstan CR, Felsenberg D, Seibel MJ (2007) Therapy insight: the risks and benefits of bisphosphonates for the treatment of tumor-induced bone disease. Nat Clin Pract Oncol 4: 42-55.

85. (2007) American Association of Oral and Maxillofacial Surgeons position paper on bisphosphonate-related osteonecrosis of the jaws. J Oral Maxillofac Surg 65: 369-376.

86. Bamias A, Kastritis E, Bamia C, Moulopoulos LA, Melakopoulos I, et al. (2005) Osteonecrosis of the jaw in cancer after treatment with bisphosphonates: incidence and risk factors. J Clin Oncol 23: 8580-8587.

87. Dimopoulos MA, Kastritis E, Moulopoulos LA, Melakopoulos I, Anagnostopoulos A, et al. (2005) The Incidence of Osteonecrosis of the Jaw (ONJ) in Patients with Multiple Myeloma Who Receive Biphosphonates Depends on the Type of Biphosphonate. 106: 637-. 
88. Hoff AO, Toth BB, Altundag K, Guarneri V, Adamus A, et al. (2006) Osteonecrosis of the jaw in patients receiving intravenous bisphosphonate therapy. 24: 8528 .

89. (2006) Dental management of patients receiving oral bisphosphonate therapy: expert panel recommendations. J Am Dent Assoc 137: 1144-1150.

90. Felsenberg D , Hoffmeister B , M A (2006) Bisphosphonattherapie assoziierte Kiefernekrosen Deutsches Arzteblatt 46: A3078- A3080.

91. Mavrokokki T, Cheng A, Stein B, Goss A (2007) Nature and Frequency of Bisphosphonate-Associated Osteonecrosis of the Jaws in Australia. Journal of Oral and Maxillofacial Surgery 65: 415-423.

92. Hellstein JW, Adler RA, Edwards B, Jacobsen PL, Kalmar JR, et al. (2011) Managing the care of patients receiving antiresorptive therapy for prevention and treatment of osteoporosis: executive summary of recommendations from the American Dental Association Council on Scientific Affairs. Journal of the American Dental Association 142: 1243-1251.

93. Lo JC, O'Ryan FS, Gordon NP, Yang J, Hui RL, et al. (2010) Prevalence of osteonecrosis of the jaw in patients with oral bisphosphonate exposure. Journal of oral and maxillofacial surgery : official journal of the American Association of Oral and Maxillofacial Surgeons 68: 243-253.

94. Dimopoulos MA, Kastritis E, Anagnostopoulos A, Melakopoulos I, Gika D, et al. (2006) Osteonecrosis of the jaw in patients with multiple myeloma treated with bisphosphonates: evidence of increased risk after treatment with zoledronic acid. Haematologica 91: 968-971.

95. Vahtsevanos K, Kyrgidis A, Verrou E, Katodritou E, Triaridis S, et al. (2009) Longitudinal cohort study of risk factors in cancer patients of bisphosphonaterelated osteonecrosis of the jaw. J Clin Oncol 27: 5356-5362.

96. Walter C, Al-Nawas B, Grotz KA, Thomas C, Thuroff JW, et al. (2008) Prevalence and risk factors of bisphosphonate-associated osteonecrosis of the jaw in prostate cancer patients with advanced disease treated with zoledronate. European urology 54: 1066-1072.

97. Hoff AO, Toth BB, Altundag K, Johnson MM, Warneke CL, et al. (2008) Frequency and risk factors associated with osteonecrosis of the jaw in cancer patients treated with intravenous bisphosphonates. J Bone Miner Res 23: 826836.

98. Froelich K, Radeloff A, Kohler C, Mlynski R, Muller J, et al. (2011) Bisphosphonate-induced osteonecrosis of the external ear canal: a retrospective study. European archives of oto-rhino-laryngology : official journal of the European Federation of Oto-Rhino-Laryngological Societies 268: 1219-1225.

99. Polizzotto MN, Cousins V, Schwarer AP (2006) Bisphosphonate-associated osteonecrosis of the auditory canal. British journal of haematology 132: 114.

100. Lenart BA, Lorich DG, Lane JM (2008) Atypical fractures of the femoral diaphysis in postmenopausal women taking alendronate. The New England journal of medicine 358: 1304-1306. 
101. Lenart BA, Neviaser AS, Lyman S, Chang CC, Edobor-Osula F, et al. (2009) Association of low-energy femoral fractures with prolonged bisphosphonate use: a case control study. Osteoporosis international 20: 1353-1362.

102. Marx RE, Sawatari Y, Fortin M, Broumand V (2005) Bisphosphonate-induced exposed bone (osteonecrosis/osteopetrosis) of the jaws: risk factors, recognition, prevention, and treatment. J Oral Maxillofac Surg 63: 1567-1575.

103. Ruggiero SL, Mehrotra B, Rosenberg TJ, Engroff SL (2004) Osteonecrosis of the jaws associated with the use of bisphosphonates: a review of 63 cases. Journal of Oral and Maxillofacial Surgery 62: 527-534.

104. Ott SM (2005) Long-term safety of bisphosphonates. J Clin Endocrinol Metab 90: 1897-1899.

105. Hoff AO, Toth BB, Altundag K, Guarneri V, Adamus A, et al. (2006) Osteonecrosis of the jaw in patients receiving intravenous bisphosphonate therapy. Journal of Clinical Oncology (Meeting Abstracts) 24.

106. Badros A (2006) Osteonecrosis of the jaw in multiple myeloma patients: clinical features and risk factors. Journal of clinical oncology : official journal of the American Society of Clinical Oncology 24: 945-952.

107. Sarathy AP, Bourgeois SL, Jr., Goodell GG (2005) Bisphosphonate-associated osteonecrosis of the jaws and endodontic treatment: two case reports. Journal of endodontics 31: 759-763.

108. Peters E, Lovas GL, Wysocki GP (1993) Lingual mandibular sequestration and ulceration. Oral surgery, oral medicine, and oral pathology 75: 739-743.

109. Neville BW DD, Allen CM, Bouquot JE (2002) Oral and Maxillofacial Pathology; Saunders WB, editor. Philadelphia.

110. Ficarra G, Beninati F, Rubino I, Vannucchi A, Longo G, et al. (2005) Osteonecrosis of the jaws in periodontal patients with a history of bisphosphonates treatment. J Clin Periodontol 32: 1123-1128.

111. Van Poznak C, Estilo C (2006) Osteonecrosis of the jaw in cancer patients receiving IV bisphosphonates. Oncology (Williston Park) 20: 1053-1062; discussion 1065-1056.

112. Migliorati CA, Schubert MM, Peterson DE, Seneda LM (2005) Bisphosphonateassociated osteonecrosis of mandibular and maxillary bone: an emerging oral complication of supportive cancer therapy. Cancer 104: 83-93.

113. Lugassy G (2004) Severe osteomyelitis of the jaw in long-term survivors of multiple myeloma: a new clinical entity. Am J Med 117: 440-441.

114. Sedghizadeh PP, Kumar SK, Gorur A, Schaudinn C, Shuler CF, et al. (2008) Identification of microbial biofilms in osteonecrosis of the jaws secondary to bisphosphonate therapy. Journal of oral and maxillofacial surgery : official journal of the American Association of Oral and Maxillofacial Surgeons 66: 767-775.

115. Schwartz HC (1982) Osteonecrosis of the jaws: a complication of cancer chemotherapy. Head Neck Surg 4: 251-253.

116. Canalis E (2005) Mechanisms of glucocorticoid action in bone. Current osteoporosis reports 3: 98-102. 
117. Gebhard KL, Maibach HI (2001) Relationship Between Systemic Corticosteroids and Osteonecrosis. American Journal of Clinical Dermatology 2: $377-388$.

118. Anderson G, Gries M, Kurihara N, Honjo T, Anderson J, et al. (2006) Thalidomide derivative CC-4047 inhibits osteoclast formation by downregulation of PU.1. Blood 107: 3098-3105.

119. Khamaisi M, Regev E, Yarom N, Avni B, Leitersdorf E, et al. (2007) Possible association between diabetes and bisphosphonate-related jaw osteonecrosis. $\mathrm{J}$ Clin Endocrinol Metab 92: 1172-1175.

120. Marx R (2005) Bisphosphonate-induced exposed bone (osteonecrosis/osteopetrosis) of the jaw: risk factors, recognition, prevention, and treatment. Journal of oral and maxillofacial surgery : official journal of the American Association of Oral and Maxillofacial Surgeons 63: 1567-1575.

121. Boonyapakorn T, Schirmer I, Reichart PA, Sturm I, Massenkeil G (2008) Bisphosphonate-induced osteonecrosis of the jaws: prospective study of 80 patients with multiple myeloma and other malignancies. Oral oncology 44: 857-869.

122. Aghaloo TL, Kang B, Sung EC, Shoff M, Ronconi M, et al. (2011) Periodontal disease and bisphosphonates induce osteonecrosis of the jaws in the rat. Journal of bone and mineral research : the official journal of the American Society for Bone and Mineral Research 26: 1871-1882.

123. Aguirre JI, Akhter MP, Kimmel DB, Pingel JE, Williams A, et al. (2012) Oncologic doses of Zoledronic acid induce osteonecrosis of the jaw-like lesions in rice rats (oryzomys palustris) with periodontitis. Journal of bone and mineral research : the official journal of the American Society for Bone and Mineral Research.

124. Lowik CW, van der Pluijm G, van der Wee-Pals LJ, van Treslong-De Groot HB, Bijvoet OL (1988) Migration and phenotypic transformation of osteoclast precursors into mature osteoclasts: the effect of a bisphosphonate. J Bone Miner Res 3: 185-192.

125. Tassone P, Forciniti S, Galea E, Morrone G, Turco MC, et al. (2000) Growth inhibition and synergistic induction of apoptosis by zoledronate and dexamethasone in human myeloma cell lines. Leukemia 14: 841-844.

126. Pietschmann P, Stohlawetz P, Brosch S, Steiner G, Smolen JS, et al. (1998) The effect of alendronate on cytokine production, adhesion molecule expression, and transendothelial migration of human peripheral blood mononuclear cells. Calcif Tissue Int 63: 325-330.

127. Berenson JR, Lichtenstein A, Porter L, Dimopoulos MA, Bordoni R, et al. (1996) Efficacy of pamidronate in reducing skeletal events in patients with advanced multiple myeloma. Myeloma Aredia Study Group. N Engl J Med 334: 488-493.

128. Thumbigere-Math V, Sabino MC, Gopalakrishnan R, Huckabay S, Dudek AZ, et al. (2009) Bisphosphonate-related osteonecrosis of the jaw: clinical features, risk factors, management, and treatment outcomes of 26 patients. J Oral Maxillofac Surg 67: 1904-1913. 
129. Badros A, Weikel D, Salama A, Goloubeva O, Schneider A, et al. (2006) Osteonecrosis of the jaw in multiple myeloma patients: clinical features and risk factors. J Clin Oncol 24: 945-952.

130. Durie BG, Katz M, Crowley J (2005) Osteonecrosis of the jaw and bisphosphonates. N Engl J Med 353: 99-102; discussion 199-102.

131. Tosi P, Zamagni E, Cangini D, Tacchetti P, Di Raimondo F, et al. (2006) Osteonecrosis of the jaws in newly diagnosed multiple myeloma patients treated with zoledronic acid and thalidomide-dexamethasone. Blood 108: 3951-3952.

132. Pozzi S, Marcheselli R, Sacchi S, Baldini L, Angrilli F, et al. (2007) Bisphosphonate-associated osteonecrosis of the jaw: a review of 35 cases and an evaluation of its frequency in multiple myeloma patients. Leuk Lymphoma 48: 56-64.

133. Wessel JH, Dodson TB, Zavras AI (2008) Zoledronate, smoking, and obesity are strong risk factors for osteonecrosis of the jaw: a case-control study. J Oral Maxillofac Surg 66: 625-631.

134. Ahmed LA, Schirmer H, Berntsen GK, Fonnebo V, Joakimsen RM (2006) Selfreported diseases and the risk of non-vertebral fractures: the Tromso study. Osteoporos Int 17: 46-53.

135. Vestergaard P, Mosekilde L (2002) Fractures in patients with hyperthyroidism and hypothyroidism: a nationwide follow-up study in 16,249 patients. Thyroid 12: 411-419.

136. Vestergaard P, Rejnmark L, Mosekilde L (2005) Influence of hyper- and hypothyroidism, and the effects of treatment with antithyroid drugs and levothyroxine on fracture risk. Calcif Tissue Int 77: 139-144.

137. Feitosa Dda S, Bezerra Bde B, Ambrosano GM, Nociti FH, Casati MZ, et al. (2008) Thyroid hormones may influence cortical bone healing around titanium implants: a histometric study in rats. J Periodontol 79: 881-887.

138. Natori J, Shimizu K, Nagahama M, Tanaka S (1999) The influence of hypothyroidism on wound healing. An experimental study. Nippon Ika Daigaku Zasshi 66: 176-180.

139. Michalowicz BS, Aeppli DP, Kuba RK, Bereuter JE, Conry JP, et al. (1991) A twin study of genetic variation in proportional radiographic alveolar bone height. J Dent Res 70: 1431-1435.

140. Conover WJ, Iman RL (1981) Rank Transformations as a Bridge between Parametric and Nonparametric Statistics. American Statistician 35: 124-129.

141. Wei X, Pushalkar S, Estilo C, Wong C, Farooki A, et al. (2012) Molecular profiling of oral microbiota in jawbone samples of bisphosphonate-related osteonecrosis of the jaw. Oral diseases 18: 602-612.

142. Mawardi H, Giro G, Kajiya M, Ohta K, Almazrooa S, et al. (2011) A role of oral bacteria in bisphosphonate-induced osteonecrosis of the jaw. J Dent Res 90: 1339-1345.

143. Nair SP, Meghji S, Wilson M, Reddi K, White P, et al. (1996) Bacterially induced bone destruction: mechanisms and misconceptions. Infection and immunity 64: 2371-2380. 
144. Reid IR, Cornish J (2012) Epidemiology and pathogenesis of osteonecrosis of the jaw. Nature reviews Rheumatology 8: 90-96.

145. Strittmatter EJ, Keller DL, LaBounty GL, Lewis DM, Graham GD (1989) The relationship between radionuclide bone scans and dental examinations. Oral surgery, oral medicine, and oral pathology 68: 576-581.

146. Arias JA, Pardo C, Olmos A, Cuadrado ML, Ruibal A (2004) Dental diseases and radionuclide imaging of the jaws. Nuclear medicine communications 25: 305-310.

147. Tang Y, Wu X, Lei W, Pang L, Wan C, et al. (2009) TGF-betal-induced migration of bone mesenchymal stem cells couples bone resorption with formation. Nature medicine 15: 757-765.

148. Kos M, Kuebler JF, Luczak K, Engelke W (2010) Bisphosphonate-related osteonecrosis of the jaws: a review of 34 cases and evaluation of risk. Journal of cranio-maxillo-facial surgery : official publication of the European Association for Cranio-Maxillo-Facial Surgery 38: 255-259.

149. Ganguli A, Steward C, Butler SL, Philips GJ, Meikle ST, et al. (2005) Bacterial adhesion to bisphosphonate coated hydroxyapatite. Journal of materials science Materials in medicine 16: 283-287.

150. Roelofs AJ, Coxon FP, Ebetino FH, Lundy MW, Henneman ZJ, et al. (2010) Fluorescent risedronate analogues reveal bisphosphonate uptake by bone marrow monocytes and localization around osteocytes in vivo. Journal of bone and mineral research : the official journal of the American Society for Bone and Mineral Research 25: 606-616.

151. Carmagnola D, Celestino S, Abati S (2008) Dental and periodontal history of oncologic patients on parenteral bisphosphonates with or without osteonecrosis of the jaws: a pilot study. Oral surgery, oral medicine, oral pathology, oral radiology, and endodontics 106: e10-15.

152. Lopez NJ, Socransky SS, Da Silva I, Japlit MR, Haffajee AD (2006) Effects of metronidazole plus amoxicillin as the only therapy on the microbiological and clinical parameters of untreated chronic periodontitis. J Clin Periodontol 33: $648-660$.

153. Epstein JB, Stevenson-Moore P (2001) Periodontal disease and periodontal management in patients with cancer. Oral oncology 37: 613-619.

154. Genco RJ (1996) Current view of risk factors for periodontal diseases. Journal of periodontology 67: 1041-1049.

155. Thumbigere-Math V, Tu L, Huckabay S, Dudek AZ, Lunos S, et al. (2012) A retrospective study evaluating frequency and risk factors of osteonecrosis of the jaw in 576 cancer patients receiving intravenous bisphosphonates. American journal of clinical oncology 35: 386-392.

156. Dimopoulos MA, Kastritis E, Bamia C, Melakopoulos I, Gika D, et al. (2009) Reduction of osteonecrosis of the jaw (ONJ) after implementation of preventive measures in patients with multiple myeloma treated with zoledronic acid. Annals of oncology : official journal of the European Society for Medical Oncology / ESMO 20: 117-120.

157. Ripamonti CI, Maniezzo M, Campa T, Fagnoni E, Brunelli C, et al. (2009) Decreased occurrence of osteonecrosis of the jaw after implementation of 
dental preventive measures in solid tumour patients with bone metastases treated with bisphosphonates. The experience of the National Cancer Institute of Milan. Annals of oncology : official journal of the European Society for Medical Oncology / ESMO 20: 137-145.

158. Montefusco V, Gay F, Spina F, Miceli R, Maniezzo M, et al. (2008) Antibiotic prophylaxis before dental procedures may reduce the incidence of osteonecrosis of the jaw in patients with multiple myeloma treated with bisphosphonates. Leukemia \& lymphoma 49: 2156-2162.

159. Edwards BJ, Hellstein JW, Jacobsen PL, Kaltman S, Mariotti A, et al. (2008) Updated recommendations for managing the care of patients receiving oral bisphosphonate therapy: an advisory statement from the American Dental Association Council on Scientific Affairs. Journal of the American Dental Association 139: 1674-1677.

160. Bedogni A, Blandamura S, Lokmic Z, Palumbo C, Ragazzo M, et al. (2008) Bisphosphonate-associated jawbone osteonecrosis: a correlation between imaging techniques and histopathology. Oral surgery, oral medicine, oral pathology, oral radiology, and endodontics 105: 358-364.

161. Xie H, Onsongo G, Popko J, de Jong EP, Cao J, et al. (2008) Proteomics analysis of cells in whole saliva from oral cancer patients via value-added three-dimensional peptide fractionation and tandem mass spectrometry. Molecular \& cellular proteomics : MCP 7: 486-498.

162. Wisniewski JR, Zougman A, Nagaraj N, Mann M (2009) Universal sample preparation method for proteome analysis. Nature methods 6: 359-362.

163. Ross PL, Huang YN, Marchese JN, Williamson B, Parker K, et al. (2004) Multiplexed protein quantitation in Saccharomyces cerevisiae using aminereactive isobaric tagging reagents. Molecular \& cellular proteomics : MCP 3: 1154-1169.

164. Bandhakavi S, Van Riper SK, Tawfik PN, Stone MD, Haddad T, et al. (2011) Hexapeptide libraries for enhanced protein PTM identification and relative abundance profiling in whole human saliva. Journal of proteome research 10 : 1052-1061.

165. Olsen JV, Schwartz JC, Griep-Raming J, Nielsen ML, Damoc E, et al. (2009) A dual pressure linear ion trap Orbitrap instrument with very high sequencing speed. Molecular \& cellular proteomics : MCP 8: 2759-2769.

166. Onsongo G, Stone MD, Van Riper SK, Chilton J, Wu B, et al. (2010) LTQiQuant: A freely available software pipeline for automated and accurate protein quantification of isobaric tagged peptide data from LTQ instruments. Proteomics 10: 3533-3538.

167. Basi DL, Hughes PJ, Thumbigere-Math V, Sabino M, Mariash A, et al. (2011) Matrix metalloproteinase-9 expression in alveolar extraction sockets of zoledronic Acid-treated rats. Journal of oral and maxillofacial surgery : official journal of the American Association of Oral and Maxillofacial Surgeons 69: 2698-2707.

168. Salo T, Makela M, Kylmaniemi M, Autio-Harmainen H, Larjava H (1994) Expression of matrix metalloproteinase-2 and -9 during early human wound 
healing. Laboratory investigation; a journal of technical methods and pathology 70: 176-182.

169. Colnot C, Thompson Z, Miclau T, Werb Z, Helms JA (2003) Altered fracture repair in the absence of MMP9. Development 130: 4123-4133.

170. Vu TH, Shipley JM, Bergers G, Berger JE, Helms JA, et al. (1998) MMP9/gelatinase $B$ is a key regulator of growth plate angiogenesis and apoptosis of hypertrophic chondrocytes. Cell 93: 411-422.

171. Murphy G, Knauper V, Atkinson S, Butler G, English W, et al. (2002) Matrix metalloproteinases in arthritic disease. Arthritis research 4 Suppl 3: S39-49.

172. Sternlicht MD, Werb Z (2001) How matrix metalloproteinases regulate cell behavior. Annual review of cell and developmental biology 17: 463-516.

173. Okada Y, Naka K, Kawamura K, Matsumoto T, Nakanishi I, et al. (1995) Localization of matrix metalloproteinase 9 (92-kilodalton gelatinase/type IV collagenase $=$ gelatinase $\mathrm{B}$ ) in osteoclasts: implications for bone resorption. Laboratory investigation; a journal of technical methods and pathology 72 : 311-322.

174. Angelov N, Moutsopoulos N, Jeong MJ, Nares S, Ashcroft G, et al. (2004) Aberrant mucosal wound repair in the absence of secretory leukocyte protease inhibitor. Thrombosis and haemostasis 92: 288-297.

175. Giraudo E, Inoue M, Hanahan D (2004) An amino-bisphosphonate targets MMP-9-expressing macrophages and angiogenesis to impair cervical carcinogenesis. The Journal of clinical investigation 114: 623-633.

176. Heikkila P, Teronen O, Moilanen M, Konttinen YT, Hanemaaijer R, et al. (2002) Bisphosphonates inhibit stromelysin-1 (MMP-3), matrix metalloelastase (MMP-12), collagenase-3 (MMP-13) and enamelysin (MMP-20), but not urokinase-type plasminogen activator, and diminish invasion and migration of human malignant and endothelial cell lines. Anti-cancer drugs 13: 245-254.

177. Allam E, Allen M, Chu TM, Ghoneima A, Jack Windsor L (2011) In vivo effects of zoledronic acid on oral mucosal epithelial cells. Oral diseases 17: 291-297.

178. McGrath JA (2005) Inherited disorders of desmosomes. The Australasian journal of dermatology 46: 221-229.

179. Jonkman MF, Pasmooij AM, Pasmans SG, van den Berg MP, Ter Horst HJ, et al. (2005) Loss of desmoplakin tail causes lethal acantholytic epidermolysis bullosa. American journal of human genetics 77: 653-660.

180. McGrath JA, McMillan JR, Shemanko CS, Runswick SK, Leigh IM, et al. (1997) Mutations in the plakophilin 1 gene result in ectodermal dysplasia/skin fragility syndrome. Nature genetics 17: 240-244.

181. Robinson ND, Hashimoto T, Amagai M, Chan LS (1999) The new pemphigus variants. Journal of the American Academy of Dermatology 40: 649-671; quiz 672-643.

182. Hatzfeld M (2007) Plakophilins: Multifunctional proteins or just regulators of desmosomal adhesion? Biochimica et biophysica acta 1773: 69-77.

183. Bierkamp C, McLaughlin KJ, Schwarz H, Huber O, Kemler R (1996) Embryonic heart and skin defects in mice lacking plakoglobin. Developmental biology 180: 780-785. 
184. Landesberg R, Cozin M, Cremers S, Woo V, Kousteni S, et al. (2008) Inhibition of oral mucosal cell wound healing by bisphosphonates. Journal of oral and maxillofacial surgery : official journal of the American Association of Oral and Maxillofacial Surgeons 66: 839-847.

185. Kim RH, Lee RS, Williams D, Bae S, Woo J, et al. (2011) Bisphosphonates induce senescence in normal human oral keratinocytes. J Dent Res 90: 810816.

186. Watts NB (1999) Clinical utility of biochemical markers of bone remodeling. Clinical chemistry 45: 1359-1368.

187. Yoshida H, Hayashi S, Kunisada T, Ogawa M, Nishikawa S, et al. (1990) The murine mutation osteopetrosis is in the coding region of the macrophage colony stimulating factor gene. Nature 345: 442-444.

188. Kong YY, Yoshida H, Sarosi I, Tan HL, Timms E, et al. (1999) OPGL is a key regulator of osteoclastogenesis, lymphocyte development and lymph-node organogenesis. Nature 397: 315-323.

189. Sheu TJ, Schwarz EM, Martinez DA, O'Keefe RJ, Rosier RN, et al. (2003) A phage display technique identifies a novel regulator of cell differentiation. The Journal of biological chemistry 278: 438-443.

190. Aghaloo TL, Felsenfeld AL, Tetradis S (2010) Osteonecrosis of the jaw in a patient on Denosumab. Journal of oral and maxillofacial surgery : official journal of the American Association of Oral and Maxillofacial Surgeons 68: 959-963.

191. Bartl R (2006) Bisphosphonat-Manual. Nature Publishing Group. pp. 83-88.

192. Jelkmann W (2001) Pitfalls in the measurement of circulating vascular endothelial growth factor. Clinical chemistry 47: 617-623.

193. Rosen HN, Moses AC, Garber J, Iloputaife ID, Ross DS, et al. (2000) Serum CTX: a new marker of bone resorption that shows treatment effect more often than other markers because of low coefficient of variability and large changes with bisphosphonate therapy. Calcif Tissue Int 66: 100-103.

194. Garnero P, Delmas PD (2004) Noninvasive techniques for assessing skeletal changes in inflammatory arthritis: bone biomarkers. Current opinion in rheumatology 16: 428-434.

195. Brown JE, Cook RJ, Major P, Lipton A, Saad F, et al. (2005) Bone turnover markers as predictors of skeletal complications in prostate cancer, lung cancer, and other solid tumors. Journal of the National Cancer Institute 97: 59-69.

196. Terpos E, Politou M, Rahemtulla A (2005) The role of markers of bone remodeling in multiple myeloma. Blood reviews 19: 125-142.

197. Christgau S, Rosenquist C, Alexandersen P, Bjarnason NH, Ravn P, et al. (1998) Clinical evaluation of the Serum CrossLaps One Step ELISA, a new assay measuring the serum concentration of bone-derived degradation products of type I collagen C-telopeptides. Clinical chemistry 44: 2290-2300.

198. Marx RE, Cillo JE, Jr., Ulloa JJ (2007) Oral bisphosphonate-induced osteonecrosis: risk factors, prediction of risk using serum CTX testing, prevention, and treatment. Journal of oral and maxillofacial surgery : official journal of the American Association of Oral and Maxillofacial Surgeons 65: 2397-2410. 
199. Kwon YD, Kim DY, Ohe JY, Yoo JY, Walter C (2009) Correlation between serum C-terminal cross-linking telopeptide of type I collagen and staging of oral bisphosphonate-related osteonecrosis of the jaws. Journal of oral and maxillofacial surgery : official journal of the American Association of Oral and Maxillofacial Surgeons 67: 2644-2648.

200. Lazarovici TS, Mesilaty-Gross S, Vered I, Pariente C, Kanety H, et al. (2010) Serologic bone markers for predicting development of osteonecrosis of the jaw in patients receiving bisphosphonates. Journal of oral and maxillofacial surgery : official journal of the American Association of Oral and Maxillofacial Surgeons 68: 2241-2247.

201. Kunchur R, Need A, Hughes T, Goss A (2009) Clinical investigation of Cterminal cross-linking telopeptide test in prevention and management of bisphosphonate-associated osteonecrosis of the jaws. Journal of oral and maxillofacial surgery : official journal of the American Association of Oral and Maxillofacial Surgeons 67: 1167-1173.

202. Flichy-Fernandez AJ, Alegre-Domingo T, Gonzalez-Lemonnier S, BalaguerMartinez J, Penarrocha-Diago M, et al. (2012) Study of serum CTX in 50 oral surgical patients treated with oral bisphosphonates. Medicina oral, patologia oral y cirugia bucal 17: e367-370.

203. Bagan JV, Jimenez Y, Gomez D, Sirera R, Poveda R, et al. (2008) Collagen telopeptide (serum CTX) and its relationship with the size and number of lesions in osteonecrosis of the jaws in cancer patients on intravenous bisphosphonates. Oral oncology 44: 1088-1089.

204. Rosen LS, Gordon D, Tchekmedyian NS, Yanagihara R, Hirsh V, et al. (2004) Long-term efficacy and safety of zoledronic acid in the treatment of skeletal metastases in patients with nonsmall cell lung carcinoma and other solid tumors: a randomized, Phase III, double-blind, placebo-controlled trial. Cancer 100: 2613-2621.

205. Bone HG (2004) Ten years' experience with alendronate for osteoporosis in postmenopausal women. The New England journal of medicine 350: 11891199.

206. Black DM, Schwartz AV, Ensrud KE, Cauley JA, Levis S, et al. (2006) Effects of continuing or stopping alendronate after 5 years of treatment: the Fracture Intervention Trial Long-term Extension (FLEX): a randomized trial. JAMA : the journal of the American Medical Association 296: 2927-2938.

207. Bolland MJ, Grey A, Horne AM, Briggs SE, Thomas MG, et al. (2012) Effects of intravenous zoledronate on bone turnover and bone density persist for at least five years in HIV-infected men. J Clin Endocrinol Metab 97: 1922-1928.

208. Grey A, Bolland MJ, Horne A, Wattie D, House M, et al. (2012) Five years of anti-resorptive activity after a single dose of zoledronate--results from a randomized double-blind placebo-controlled trial. Bone 50: 1389-1393.

209. Kwon YD, Ohe JY, Kim DY, Chung DJ, Park YD (2011) Retrospective study of two biochemical markers for the risk assessment of oral bisphosphonaterelated osteonecrosis of the jaws: can they be utilized as risk markers? Clinical oral implants research 22: 100-105. 
210. Lehrer S, Montazem A, Ramanathan L, Pessin-Minsley M, Pfail J, et al. (2009) Bisphosphonate-induced osteonecrosis of the jaws, bone markers, and a hypothesized candidate gene. Journal of oral and maxillofacial surgery : official journal of the American Association of Oral and Maxillofacial Surgeons 67: 159-161.

211. Raje N, Woo SB, Hande K, Yap JT, Richardson PG, et al. (2008) Clinical, radiographic, and biochemical characterization of multiple myeloma patients with osteonecrosis of the jaw. Clinical cancer research : an official journal of the American Association for Cancer Research 14: 2387-2395.

212. Boissier S, Colombel M, Delmas P, Cabon C, P C (2002) Zoledronic acid inhibits in vivo experimental angiogenesis (abstract B3). Bone: 30:38S.

213. Wood J (2002) Novel antiangiogenic effects of the bisphosphonate compound zoledronic acid. The Journal of pharmacology and experimental therapeutics 302: 1055-1061.

214. Ferretti G, Fabi A, Carlini P, Papaldo P, Cordiali Fei P, et al. (2005) Zoledronicacid-induced circulating level modifications of angiogenic factors, metalloproteinases and proinflammatory cytokines in metastatic breast cancer patients. Oncology 69: 35-43.

215. Ferrara N, Davis-Smyth T (1997) The biology of vascular endothelial growth factor. Endocrine reviews 18: 4-25.

216. Street J, Bao M, deGuzman L, Bunting S, Peale FV, Jr., et al. (2002) Vascular endothelial growth factor stimulates bone repair by promoting angiogenesis and bone turnover. Proceedings of the National Academy of Sciences of the United States of America 99: 9656-9661.

217. Gerber HP, Vu TH, Ryan AM, Kowalski J, Werb Z, et al. (1999) VEGF couples hypertrophic cartilage remodeling, ossification and angiogenesis during endochondral bone formation. Nature medicine 5: 623-628.

218. Wehrhan F, Stockmann P, Nkenke E, Schlegel KA, Guentsch A, et al. (2011) Differential impairment of vascularization and angiogenesis in bisphosphonate-associated osteonecrosis of the jaw-related mucoperiosteal tissue. Oral surgery, oral medicine, oral pathology, oral radiology, and endodontics 112: 216-221.

219. Arduino PG, Menegatti E, Scoletta M, Battaglio C, Mozzati M, et al. (2011) Vascular endothelial growth factor genetic polymorphisms and haplotypes in female patients with bisphosphonate-related osteonecrosis of the jaws. Journal of oral pathology \& medicine : official publication of the International Association of Oral Pathologists and the American Academy of Oral Pathology 40: 510-515.

220. Christodoulou C, Pervena A, Klouvas G, Galani E, Falagas ME, et al. (2009) Combination of bisphosphonates and antiangiogenic factors induces osteonecrosis of the jaw more frequently than bisphosphonates alone. Oncology 76: 209-211.

221. Estilo CL, Fornier M, Farooki A, Carlson D, Bohle G, 3rd, et al. (2008) Osteonecrosis of the jaw related to bevacizumab. Journal of clinical oncology : official journal of the American Society of Clinical Oncology 26: 40374038 . 
222. Disel U, Besen AA, Ozyilkan O, Er E, Canpolat T (2012) A case report of bevacizumab-related osteonecrosis of the jaw: old problem, new culprit. Oral oncology 48: e2-3.

223. Koch FP, Walter C, Hansen T, Jager E, Wagner W (2011) Osteonecrosis of the jaw related to sunitinib. Oral and maxillofacial surgery 15: 63-66.

224. Fleissig Y, Regev E, Lehman H (2012) Sunitinib related osteonecrosis of jaw: a case report. Oral surgery, oral medicine, oral pathology and oral radiology 113: e1-3.

225. Roskoski R, Jr. (2007) Sunitinib: a VEGF and PDGF receptor protein kinase and angiogenesis inhibitor. Biochemical and biophysical research communications 356: 323-328.

226. Ferrara N, Hillan KJ, Novotny W (2005) Bevacizumab (Avastin), a humanized anti-VEGF monoclonal antibody for cancer therapy. Biochemical and biophysical research communications 333: 328-335.

227. Santini D, Vincenzi B, Dicuonzo G, Avvisati G, Massacesi C, et al. (2003) Zoledronic acid induces significant and long-lasting modifications of circulating angiogenic factors in cancer patients. Clinical cancer research : an official journal of the American Association for Cancer Research 9: 28932897.

228. Hasmim M, Bieler G, Ruegg C (2007) Zoledronate inhibits endothelial cell adhesion, migration and survival through the suppression of multiple, prenylation-dependent signaling pathways. Journal of thrombosis and haemostasis : JTH 5: 166-173.

229. Bezzi M, Hasmim M, Bieler G, Dormond O, Ruegg C (2003) Zoledronate sensitizes endothelial cells to tumor necrosis factor-induced programmed cell death: evidence for the suppression of sustained activation of focal adhesion kinase and protein kinase B/Akt. The Journal of biological chemistry 278: 43603-43614.

230. Stylianou K, Lioudaki E, Papadimitraki E, Kokologiannakis G, Kroustalakis N, et al. (2011) Crescentic glomerulonephritis associated with vascular endothelial growth factor (VEGF) inhibitor and bisphosphonate administration. Nephrology, dialysis, transplantation : official publication of the European Dialysis and Transplant Association - European Renal Association 26: 1742-1745.

231. Khosla S, Burr D, Cauley J, Dempster DW, Ebeling PR, et al. (2008) Oral bisphosphonate-induced osteonecrosis: risk factors, prediction of risk using serum CTX testing, prevention, and treatment. Journal of oral and maxillofacial surgery : official journal of the American Association of Oral and Maxillofacial Surgeons 66: 1320-1321; author reply 1321-1322.

232. Schwartz HC (2008) Serum CTX testing. Journal of oral and maxillofacial surgery : official journal of the American Association of Oral and Maxillofacial Surgeons 66: 1319-1320; author reply 1320.

233. Boivin GY (2000) Alendronate increases bone strength by increasing the mean degree of mineralization of bone tissue in osteoporotic women. Bone 27: 687694. 
234. Chavassieux PM, Arlot ME, Reda C, Wei L, Yates AJ, et al. (1997) Histomorphometric assessment of the long-term effects of alendronate on bone quality and remodeling in patients with osteoporosis. The Journal of clinical investigation 100: 1475-1480.

235. Akkus O, Polyakova-Akkus A, Adar F, Schaffler MB (2003) Aging of microstructural compartments in human compact bone. Journal of bone and mineral research : the official journal of the American Society for Bone and Mineral Research 18: 1012-1019.

236. Ciarelli TE, Fyhrie DP, Parfitt AM (2003) Effects of vertebral bone fragility and bone formation rate on the mineralization levels of cancellous bone from white females. Bone 32: 311-315.

237. Schaffler MB, Radin EL, Burr DB (1989) Mechanical and morphological effects of strain rate on fatigue of compact bone. Bone 10: 207-214.

238. Pozzi S, Vallet S, Mukherjee S, Cirstea D, Vaghela N, et al. (2009) High-dose zoledronic acid impacts bone remodeling with effects on osteoblastic lineage and bone mechanical properties. Clinical cancer research : an official journal of the American Association for Cancer Research 15: 5829-5839.

239. Allen MR, Iwata K, Phipps R, Burr DB (2006) Alterations in canine vertebral bone turnover, microdamage accumulation, and biomechanical properties following 1-year treatment with clinical treatment doses of risedronate or alendronate. Bone 39: 872-879.

240. Allen MR, Turek JJ, Phipps RJ, Burr DB (2011) Greater magnitude of turnover suppression occurs earlier after treatment initiation with risedronate than alendronate. Bone 49: 128-132.

241. Viguet-Carrin S, Garnero P, Delmas PD (2006) The role of collagen in bone strength. Osteoporosis international : a journal established as result of cooperation between the European Foundation for Osteoporosis and the National Osteoporosis Foundation of the USA 17: 319-336.

242. Ruppel ME, Miller LM, Burr DB (2008) The effect of the microscopic and nanoscale structure on bone fragility. Osteoporosis international : a journal established as result of cooperation between the European Foundation for Osteoporosis and the National Osteoporosis Foundation of the USA 19: 12511265.

243. Gourion-Arsiquaud S, Allen MR, Burr DB, Vashishth D, Tang SY, et al. (2010) Bisphosphonate treatment modifies canine bone mineral and matrix properties and their heterogeneity. Bone 46: 666-672.

244. Allen MR, Kubek DJ, Burr DB (2010) Cancer treatment dosing regimens of zoledronic acid result in near-complete suppression of mandible intracortical bone remodeling in beagle dogs. Journal of bone and mineral research : the official journal of the American Society for Bone and Mineral Research 25: 98-105.

245. Huja SS, Fernandez SA, Hill KJ, Li Y (2006) Remodeling dynamics in the alveolar process in skeletally mature dogs. The anatomical record Part A, Discoveries in molecular, cellular, and evolutionary biology 288: 1243-1249.

246. Major P, Lortholary A, Hon J, Abdi E, Mills G, et al. (2001) Zoledronic acid is superior to pamidronate in the treatment of hypercalcemia of malignancy: a 
pooled analysis of two randomized, controlled clinical trials. Journal of clinical oncology : official journal of the American Society of Clinical Oncology 19: 558-567.

247. Frost HM (1998) A brief review for orthopedic surgeons: fatigue damage (microdamage) in bone (its determinants and clinical implications). Journal of orthopaedic science : official journal of the Japanese Orthopaedic Association 3: 272-281.

248. Reilly GC, Currey JD (2000) The effects of damage and microcracking on the impact strength of bone. Journal of biomechanics 33: 337-343.

249. Carter DR, Hayes WC (1977) Compact bone fatigue damage--I. Residual strength and stiffness. Journal of biomechanics 10: 325-337.

250. Mashiba T, Hirano T, Turner CH, Forwood MR, Johnston CC, et al. (2000) Suppressed bone turnover by bisphosphonates increases microdamage accumulation and reduces some biomechanical properties in dog rib. Journal of bone and mineral research : the official journal of the American Society for Bone and Mineral Research 15: 613-620.

251. Hoefert S, Schmitz I, Tannapfel A, Eufinger H (2010) Importance of microcracks in etiology of bisphosphonate-related osteonecrosis of the jaw: a possible pathogenetic model of symptomatic and non-symptomatic osteonecrosis of the jaw based on scanning electron microscopy findings. Clinical oral investigations 14: 271-284.

252. Li J, Mashiba T, Burr DB (2001) Bisphosphonate treatment suppresses not only stochastic remodeling but also the targeted repair of microdamage. Calcif Tissue Int 69: 281-286.

253. Lenart BA, Neviaser AS, Lyman S, Chang CC, Edobor-Osula F, et al. (2009) Association of low-energy femoral fractures with prolonged bisphosphonate use: a case control study. Osteoporosis international : a journal established as result of cooperation between the European Foundation for Osteoporosis and the National Osteoporosis Foundation of the USA 20: 1353-1362.

254. Russell RG, Rogers MJ (1999) Bisphosphonates: from the laboratory to the clinic and back again. Bone 25: 97-106.

255. Ribatti D, Nico B, Mangieri D, Maruotti N, Longo V, et al. (2007) Neridronate inhibits angiogenesis in vitro and in vivo. Clin Rheumatol 26: 1094-1098.

256. Ruggiero SL, Mehrotra B, Rosenberg TJ, Engroff SL (2004) Osteonecrosis of the jaws associated with the use of bisphosphonates: a review of 63 cases. J Oral Maxillofac Surg 62: 527-534.

257. Bedogni A, Blandamura S, Lokmic Z, Palumbo C, Ragazzo M, et al. (2008) Bisphosphonate-associated jawbone osteonecrosis: a correlation between imaging techniques and histopathology. Oral Surg Oral Med Oral Pathol Oral Radiol Endod 105: 358-364.

258. Khosla S, Burr D, Cauley J, Dempster DW, Ebeling PR, et al. (2007) Bisphosphonate-associated osteonecrosis of the jaw: report of a task force of the American Society for Bone and Mineral Research. J Bone Miner Res 22: 1479-1491.

259. Team RDC (2008) R: A Language and Environment for Statistical Computing. Vienna, Austria: R Foundation for Statistical Computing. 
260. Peters E, Lovas GL, Wysocki GP (1993) Lingual mandibular sequestration and ulceration. Oral Surg Oral Med Oral Pathol 75: 739-743.

261. Ruggiero S, Gralow J, Marx RE, Hoff AO, Schubert MM, et al. (2006) Practical Guidelines for the Prevention, Diagnosis, and Treatment of Osteonecrosis of the Jaw in Patients With Cancer. pp. 7-14.

262. Assouline-Dayan Y, Chang C, Greenspan A, Shoenfeld Y, Gershwin ME (2002) Pathogenesis and natural history of osteonecrosis. Semin Arthritis Rheum 32: 94-124.

263. Freiberger JJ, Padilla-Burgos R, Chhoeu AH, Kraft KH, Boneta O, et al. (2007) Hyperbaric oxygen treatment and bisphosphonate-induced osteonecrosis of the jaw: a case series. J Oral Maxillofac Surg 65: 1321-1327.

264. Mignogna MD, Fedele S, Lo Russo L, Ciccarelli R, Lo Muzio L (2006) Case 2. Osteonecrosis of the jaws associated with bisphosphonate therapy. J Clin Oncol 24: 1475-1477.

265. Hansen T, Kunkel M, Weber A, James Kirkpatrick C (2006) Osteonecrosis of the jaws in patients treated with bisphosphonates - histomorphologic analysis in comparison with infected osteoradionecrosis. J Oral Pathol Med 35: 155160 . 